SERVIÇO DE PÓS-GRADUAÇÃO DO ICMC-USP

Data de Depósito: $\quad 17.10 .2005$

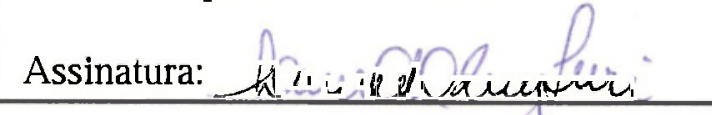

\title{
Simulação numérica de escoamentos de fluidos utilizando diferenças finitas generalizadas
}

Fernanda Olegário dos Santos

Orientador: Prof. Dr. Antomio Castelo Filho

Dissertação apresentada ao Instituto de Ciências Matemáticas e de Computação - ICMC-USP, como parte dos requisitos para obtenção do título de Mestre em Ciências - Ciências da Computação e Matemática Computacional.

USP - Säo Carlos

Outubro/2005 
Aluno: Fernanda Olegario dos Santos

\section{A Comissão Julgadora:}

Prof. Dr. Antonio Castelo Filho

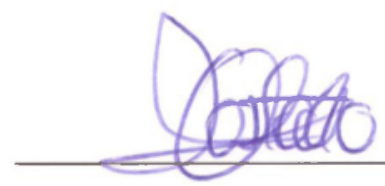

Prof. Dr. Norberto Mangiavacchi

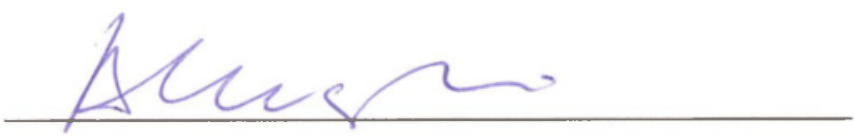

Prof. Dr. Márcio Teixeira de Mendonça

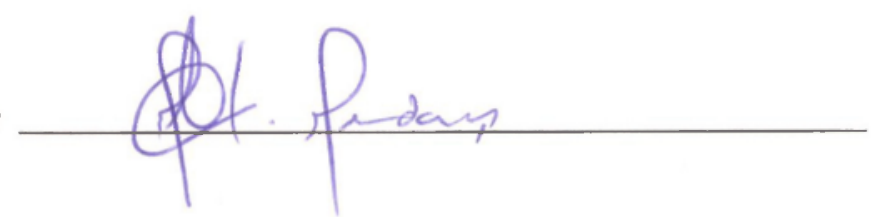


Aos meus pais Neide e David, com carinho. 


\section{Agradecimentos}

Em primeiro lugar agradeç a Deus por estar presente em todos os momentos de minha vida.

Em especial a minha mãe Neide, meu pai David e minha irmã Liliana, por sempro acreditarem em mim e no men potencial. Pelo carinho, amor, orações, dedicação e atenção. Amo muito vocês!

Ao meu sobrinho (Gabriel, pela alegria, carinho, brincadeiras ...

Ao meu namorado Magno, pelo carinho, amor, paciência, por estar sempre ao meu lado em todos os momentos.

Agradego aos meus tios Neusa e José pelo carinho e sempre incentivo.

Ao men orientador Castelo, pela orientação e apoio.

Aos professores do grupo de pesquisa, pela colaboração e convivência.

'Tambem aos jamais esquecidos amigos de graduação e professores da UFSCar, especialmente ao prof. Artur Darezzo Filho, pela orientação, apoio, amizade e incentivo durante a gradua(s̃ão.

As amigas Analice, Marcela, Gilcilene e Kémelli, pelo companheirismo e convivência, nos estudos c nos momentos de descontração.

Ao Mário, Fernando, Cássio, João Paulo, Flton, enfim aos amigos do LCAD, pola amizade e pela ajuda.

A FAPESP, pelo apoio financeiro no desenvolvimento deste trabalho.

Agradeço, por fim, a todos que direta ou indiretamentc, contribuiram para o sucesso deste trabalho. 


\section{Resumo}

Este trabalho apresenta parte de um sistema de simulação integrado para escoamento de fluido incompressivel bidimensional em malhas não estruturadas denominado LmFlow-2D. O sistema consiste de três módulos: um módulo modelador, um módulo simulador e um módulo visializador. A parte do sistema apresentado neste trabalho é o módulo simulador. Lste módulo, implementia as equações de Navier-Stokes. As equações governantes sào discretizadas pelo método de diferenças finitas generalizadas e os termos convectivos pelo método semi-lagrangeano. Um método de projeção é empregado para desacoplar as componentes da velocidade e pressão. O gerenciamento da malha não estruturada é feito pela estrutura de dados SHE. Os resultados numéricos obtidos pelo UmFlow-2D são comparados com soluçôes analíticas e solıçoes numéricas de ontros trabalhos.

Palavras-chave: Simulação numérica. Método de diferenças Finitas Generalizadas. Malhas nào estruturada. 


\section{Abstract}

This work presents an integrate simulation systom, alled UmF'low-2D, wich aims at simulating two-dimensional incompressible fluid flow using unstructed mesh. The system is divided three modules: modeling module, simulation module and visıalization module. In this work we present the simulation module. The simulation module implements the Navier-Stokes equation. The governing equations are discretized by a generalized finite difference method and the convective terms by semi-lagrangean method. A projection method is employed to uncouple the velocity componentes and pressure. The management at, the unstructed mesh is ready using a data structure called SHE. The numerical results are compared with analytical solutions and numerical simnlations of other works.

Key Words: Numerical simulation. Generalized finite difference method. Lnstructed mesh 


\section{Lista de Figuras}

1.1 Diferentes tipos de célula de nó . . . . . . . . . . . . . . . . 7

1.2 Função de aproximação $f_{0}$ : (a)uma linha reta com $s-1$, e (b) uma parábola com $s \ldots 2 \ldots \ldots \ldots \ldots \ldots \ldots \ldots$

3.1 Elemento triangular. . . . . . . . . . . . . . . 26

3.2 Tipos de células. . . . . . . . . . . . . . . . . . . . . . . . 29

1.1 Malha triangnlarizada. . . . . . . . . . . . . . 32

4.2 Cólula Computacional. . . . . . . . . . . . . . . . . 32

4.3 Célula com triângulos numerados. . . . . . . . . . . . 36

4.4 Condição de contorno: Outflow . . . . . . . . . . . . . 38

4.5 Entidades de orgarnização da estrutura de dados SHE. . . . . . . . . . . 41

j.1 Diagrama de Casos de Uso do software. . . . . . . . . . . . . . 47

6.1 Domínio para um escoamento de Hagen-Poiseuille. O escoamento é da esquerda para a direita e o comprimento do canal a $3 L \ldots \ldots$. . . . . . . 51

6.2 Malha com 183 elementos, gerada pelo Fasymesh . . . . . . . . . . . 52

6.3 Simulação numórica do escoamento de Hagen-Poiseuille com perfil reto: campo de velocidade na direção $x$, calculados na malha intermediária . . . . 53

6.4 Comparação entre soluçōes numéricas obtida pelo ambiente de simulação UmFlow-2D e a solução analítica dada pela cculação (6.11), sobre as três

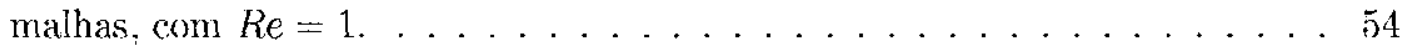

6.5 Simulação numérica do escoamento de Hagen-Poiseuille com perfil de parabólico: campo de velocidade na direção $x$, calculados na malha fina. . . . . . . . 54

6.6 Comparação entre solıçōes numéricas obtida pelo ambiente de simulação UmFlow-2D e a solıção analítica dada pela equação $(6.11)$, sobre as três malhas, com $R e=1 . \ldots \ldots \ldots \ldots . \ldots \ldots 5$

6.7 Comparação entre soluçôes numóricas obtida polo ambionte de simulação LimFlow-2D e a solução analítica dada pela equação (6.11), sobre uma malha (4.426 elementos triangulares), para $R e=1, R e=10$ e $R e=100 \ldots \ldots .55$

6.8 Geometria do problema da expansão brusca. . . . . . . . . . . . 56

6.9 Problema da expansão: campo de velocidade na dirç̧ão x. . . . . . . . 57

6.10 Problema da expansão: campo de velocidade na direção x. . . . . . . . 57

6.11 Problema da expansão: campo de velocidade na direção x. . . . . . . . 57

6.12 Placa retangular com contração. . . . . . . . . . . . . 57 
6.13 Placa retangular com contração: campo de velocidade na direção $\mathrm{x}, \mathrm{Re}=10 . \quad 58$ 6.14 Placa retangular com contração: campo de velocidade na direção y, $\mathrm{Re}=10 . \quad 58$ 6.15 Placa retangular com contração: campo de velocidade na direção $\mathrm{x}, \mathrm{Re}=100.59$ 6.16 Placa retangular com contração: campo de velocidade na direção y, Re $=100.59$ 6.17 Placa retangular com contração: vetores velocidade, Re $-100 \ldots \ldots$

6.18 Geometria do problema com geometria curva. . . . . . . . . . . . 60

6.19 Malha com 4.845 elementos, gerada pelo Easymesh. . . . . . . . . . . . . 60

6.20 Problema com geometria curva: campo de velocidade na direção $\mathrm{x}, \mathrm{Re}=1$. 61

6.21 Problema com geometria curva: campo de velocidade na direção y, $\operatorname{Re}=1$. . 61

6.22 Problema com geometria curva: campo de velocidade na direção $x, \operatorname{Re}=100.61$

6.23 Problema com geometria curva: campo de velocidade na direção $y, R e=100.62$

6.24 Problema com geometria curva: vetores velocidade, $\mathrm{Re}=1 . \ldots \ldots 2$

6.25 Problema com geometria curva: vetores velocidade, $R e=100 \ldots \ldots 2$ 


\section{Sumário}

Introdução 1

1 Método Numérico para Equações Diferenciais 4

1.1 Introdução . . . . . . . . . . . . . . . . . . . . . 4

1.2 Método de Diferenças Finitas Generalizadas utilizando Mínimos Quadrados . 4

1.2.1 Célula Computacional associada a um Nó . . . . . . . . . . 5

1.2 .2 Aproximação por Mínimos Quladrados . . . . . . . . . . . 7

2 Equações de Navier-Stokes $\quad 14$

2.1 Introdução . . . . . . . . . . . . . . . . . . . . . . . 14

2.2 Descrição do Movimento de Flıido . . . . . . . . . . . . 14

2.2 .1 Derivada Total . . . . . . . . . . . . . 15

2.3 Lquaçōes Governantes . . . . . . . . . . . . . . . . . . 16

2.3.1 Equação de Conservação de Massa . . . . . . . . . . . . 16

2.3.2 Equação do Balanço de Quantidade de Movimento . . . . . . . . 16

2.4 Lquações Governantes na Forma Bidimensional . . . . . . . . . . . . . 17

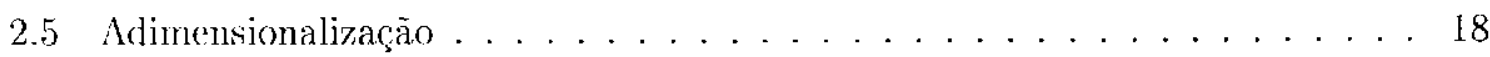

2.6 Condições Inicial e de Contorno $\ldots \ldots \ldots \ldots$

2.6 .1 Condiçoes para Contornos Rígidos . . . . . . . . . . . . 19

2.6.2 Condições de Contorno na Superfície Livre . . . . . . . . . . . . 20

2.6 .3 Condição para a Pressão . . . . . . . . . . . . . . . 21

3 Métodos Numéricos para Equação de Navier-Stokes $\quad 22$

3.1 Introdução . . . . . . . . . . . . . . . . . . . 22

3.2 A idéia Geral do Método da Projeção . . . . . . . . . . . . . 23

3.3 Método de Projeção . . . . . . . . . . . . . . . 24

3.4 Método do Passo Fracionário . . . . . . . . . . . . . 25

3.5 Método Semi-Lagrangeano . . . . . . . . . . . . . . . . . . . . . . . . . . .

3.6 Método Utilizado no UmFlow-2D . . . . . . . . . . . . . . 27

3.6 .1 Classificação das Cólulas . . . . . . . . . . . . . . . . . . . 28

3.6 .2 Condiçoes Inicial e de Contorno . . . . . . . . . . . . . . . . . . . . . . 29

3.6 .3 Condição de Fstabilidade do Método . . . . . . . . . . . . . . 29 
4 Discretização das Equações $\quad 31$

4.1 Introdução. . . . . . . . . . . . . . . . . . . . 31

4.2 Discretização das Equaçòes . . . . . . . . . . . . . . . . . . . . . 31

4.2.1 Aproximação das Volocidades . . . . . . . . . . . . . . . 33

4.2.2 Aproximação da Equação de Poisson para a Pressão . . . . . . . . . . 33

4.2 .3 Técnicas de Armazenagem . . . . . . . . . . . . . . 36

1.2.4 Aproximação da Equação de Atualização da Velocidade . . . . . . . . 37

4.2.5 Aproximação das Condiçóes de Contorno . . . . . . . . . . . . . 37

4.3 Método dos Gradientes Bi-conjugados para Sistemas Esparsos . . . . . . . 38

4.4 Estrutura de dados SHE . . . . . . . . . . . . . . . . . 10

4.4 .1 A estrutura "Singular Handle-Edge" . . . . . . . . . . . . . . . . 40

4.4 .2 Implementação . . . . . . . . . . . . . . . 41

5 Desenvolvimento do Software $\quad 42$

5.1 Considerações Iniciais . . . . . . . . . . . . . . . . . . . . . . 42

5.2 Engenharia de Software . . . . . . . . . . . . . . . . 42

5.3 O Processo de Software . . . . . . . . . . . . . . . . . 43

5.3.1 Modelos de Processos de Software . . . . . . . . . . . . . . . . . 43

5.4 Padróes de Projeto . . . . . . . . . . . . . . . . . . . 44

5.5 Documento de Requisitos . . . . . . . . . . . . . . . . 44

5.5 .1 Visão Geral do Sistema . . . . . . . . . . . . . . . . 45

5.5 .2 Diagrama de Caso de Uso . . . . . . . . . . . . . . . 47

5.6 Ambiente de Simulação UmFlow-2D . . . . . . . . . . . . . 48

5.6 .1 Modelador . . . . . . . . . . . . . . . . . . 48

5.6 .2 Simulador . . . . . . . . . . . . . . . . . 48

5.6 .3 Visualizador .......................... 18

5.6 .4 Re-inicializador . . . . . . . . . . . . . . 18

6 Resultados Numéricos e Validação do Ambiente de Simulação UmFlow-2D 50

6.1 Introdução . . . . . . . . . . . . . . . . . . . . 50

6.2 Simılação do Escoamento em um Canal . . . . . . . . . . . . . 50

6.3 Simulação nıma Expansão Brısca . . . . . . . . . . . . . . . . . 55

6.4 Simulação de uma Placa com Contração . . . . . . . . . . . . . . . 57

6.5 Simulação em domínio com Geometria Curva . . . . . . . . . . . . . . 59

Considerações Finais 


\section{Introdução}

O estudo de fenômenos físicos e leis da natureza é uma atividade que vem sendo desenvolvida há muitos séculos com diversas descobertas que motivam a comunidade científica até os dias atuais. Como ponto de partida para o estudo das leis da natureza haviam os métodos práticos ocupando-se das observaçoes e experimentos e os métodos teóricos desenvolvendo leis e teorias físicas. Com o objetivo de conectar esses dois métodos foi criado os métodos numéricos, beneficiando enormemente o desenvolvimento científico. Com o desenvolvimento de novos algoritmos, e computadores de alta velocidade e de grande capacidade de armazenamento o uso de técnicas numéricas para a solıção de problemas complexos da engenharia e da física tornou-se uma realidade. Fim função dessa disponibilidade computacional o desenvolvimento de algoritmos para a solução dos mais diversos problemas tem recebido enorme atenção dos analistas numéricos. As principais vantagens das soluções numéricas são: baixo custo, evolução temporal do processo, apresentar resultados com rapidez, resolver problemas em geometrias complexas e etc. Fntretanto, a solução numérica também apresenta algumas desvantagens, como: instabilidades, crros de truncamento, prescrição das condições de contorno apropriadas e custos computacionais.

Com o desenvolvimento de sistemas de software, surgiu a Fingenharia de Software (ES) motivando o interesse em reduzir custos e aumentar a qualidade dos softwares. Os fundamentos científicos para a ES envolvem o uso de modeks abstratos e rigorosos que permitem ao engenheiro especificar, projetar, implementar e manter sistemas de software, avaliando e garantindo suas qualidades.

Neste trabalho, a atenção é voltada para o estudo do desenvolvimento de um simulador de escoamentos de fluidos utilizando um método numérico em domínio complexo. A solução dos problemas de escoamentos de fluidos reculer o manuseio das equaçöes de Navier-Stokes, altamente não lincar acopladas às equações da conservação de massa e energia. As equaçoes de Navier-Stokes em conjunto com condições iniciais e de contorno fisicamente apropriadas, permitem em princípio, obter informaçōes do caráter fundamental da dinâmica dos flıidos, tornando a solução numérica fundamental devido às dificuldades de se obter, na maior parte dos problemas, soluções teóricas para as equaçöes de Navier-Stokes, dando início a uma importante área de esstudo dos métodos computacionais para a simulação de fenômenos que envolvem fluidos em movimento com ou sem troca de calor, chamada Dinâmica. de Fluidos Computarional (DFC). O objelivo básico da DFC ó reduzir o número de experimentos e explorar fenômenos que não poderiam ser estudados em laboratório de forma prática. Utilizando as técnicas da. DFC pode-se avaliar numericamente os diversos parâmetros relevantes do problema. Fsses podem ser facilmente alterados até que o resultado da. 
simulação atenda às exigĉncias do projeto. A DFC está envolvida, praticamente, em todos os processos de produção de energia, nos fenomenos ambientais, nos projetos de equipamentos tćrmicos, na engenharia aeronáutica e aeroespacial, engenharia de reatores, engenharia química, medicina, siderúrgica, indústria textil e injeções em moldes.

O interesse de estudo neste trabalho, são as solıçōes numéricas de escoamentos de fluidos estacionários e transientes, internos, laminares, incompressivveis c viscosos para fluidos Newtonianos. Nas últimas décadas muitos esforços têm sido dirigidos na obtenção de solıções muméricas de escoamentos de fluidos viscosos incompressíveis. A maioria desses métodos foram influenciados pelo método MAC (Marker-and-Cell) |Harlow and Welch, 1965). Métodos encontrados em |Viecelli, 1969|, |Hirt and Nichols, 1971|, |Hirt and Cook, 1972|, |Miyata and Masuko, 1985|, |Tomé and McKce, 1994| e outros, são exemplos de técnicas numéricas baseadas no método MAC.

Recentemente, o grupo de pesquisa em Matemática Aplicada do ICMC - USP, vem desenvolvendo um ambiente de simulação de escoamentos incompressíveis tridimensionais denominado FreeFlow-3D |Castelo et al., 2000|. FreeFlow-3D foi obtido pela extensão do método GENSMAC |Tomé and McKee, 1994| para problemas tridimensionais e é composto por 3 módulos: Modflow-3D - um ambiente para modelar escoamentos tridimensionais; Simflow-3D - esse módılo implementa as equações governantes juntamente com as condições iniciais e de contorno; Visflow-3D - um módulo responsável pela visualização dos resultados numéricos gerados pelo módulo Simflow-3D. Detalhes do FreeFlow-3D podem ser obtidos em |Castelo et al., 2000|.

Na prálica, muitos problemas podem ser modelados em duas dimensões, por isso uma versão bidimensional do Freeflow-3I), denominada FreeFlow-2D |Oliveira, 1999| que simula superfícies livres em geometrias complexas com malhas estruturadas, foi desenvolvida. Porém, até o momento, FreeFlow-2D pode resolver problemas bidimensionais para fluido Newtoniano, fluido Newtoniano generalizado do tipo Cross Model |Siquicri, 2002|, para escoamentos em regime turbulento |Ferreira, 2001|, |Brandi, 2005|, escoamento nâo-isotérmicos |Sabatini, 2002|, problemas de escoamentos multifásicos |Santos, 2002|, |Sousa and Mangiavacchi, 2002| e para problemas de escoamentos não-Newtonianos e viscoelásticos |Grossi, 2003|, |Silva, 2003|, |Doricio, 2003| e |Silva, 2005|. Os ambientes FreeFlow-2D e FreeFlow-3D abordam apenas domínios decompostos em malhas uniformes. Este trabalho o uma nova etapa para o ambiente de simulação FreeFlow-2D, que agora permite simulações em domínios decompostos através de malhas não estruturadas. Este novo ambiente denomina-se LmFlow-2D.

Para o desenvolvimento deste novo ambiente também é realizado o estudo do método de Diferenças Finitas Generalizadas (DFG), desenvolvido a partir do método de aproximação por mínimos quadrados localizados (MIS)' ${ }^{\prime}$, introdızido por Shepard |Shepard, 1968|, o qual fornece uma alternativa às interpolações clássicas de aproximações de funções a partir de seus valores dados em uma série de pontos distribuídos irregularmente. Este trabalho também têm como objeto de estudo o método de projeção de Chorin |Chorin, 1968| : o método semi-lagrangeano |Phillips and Williams, 2001| para os termos convectivos. Fstes métodos reunidos em ım único ambientc. de simulação numérica é uma das principais contribuições deste trabalho.

Para o gerenciameto da malha não estruturada em dıas dimensões, é utilizada a estru-

\footnotetext{
'moving least square
} 
tura de dados "Singular Handle-Edge" (SHF) |Lixier et al., 2003|.

O presente trabalho está organi\%ado da segninte mancira:

- No capítulo 2 apresenta-se as propriedades do método numérico DFG para equações diferenciais;

- No capítulo 3 a formulação matemática e as equações de Navier-Stokes são apresentadlas;

- No capítılo 4 apresenta-se o método da projeção e o mótodo utilizada no módulo simulador;

- No capítulo 5 as equaçóes utilizadas são discretizadas e é apresentada a cstrutura de dados SHE:

- No capítılo 6 apresenta-se o estudo de Engenharia de Software e o ambiente de simulação UmFlow-2D;

- No capítulo 7 tem-se a validação e os resultados do simulaçócs realizados pelo Limflow-2D;

- Por fim, tem-se as conclusões e contribuições deste trabalho; 


\section{$-I$ \\ Método Numérico para Equações Diferenciais}

\subsection{Introdução}

A modelagem matemática de problemas reais em ciências aplicadas a cada dia sc torna uma ferramenta mais útil na compreensão dos fenomenos envolvidos nesses, permitindo a simulação computacional desses problemas e revelando-se determinante no desenvolvimento de novas tecnologias. (O resultado dessa modelagem é um conjunto de equações que deve ser resolvido cm computadores, produzindo uma solução numérica, denominada simulagão e descreve propriedades do problema em questão.

A simulação numérica pode ser vista como uma relação entre resultados téricos e práticos, que muitas vezes nâo apresentat as restrições que tais resultados podem impor na modelagem da física do problema. Destia forma, para que a solução numérica seja. aplicada a um problema, expressões matemáticas devem ser derivadas. A modelagem de problemas que onvolvem escoamentos de fluidos requer solução que manuseiam as equações de Navier-Stokes.

No presente trabalho, o método de discretização utilizado co o método de Diferenças Finitas Generalizadas sobre uma malha não estruturada. Detalhes das propriedades de métodos numéricos para EIDP's podem ser encontrados cm |Lapidus and Pinder, 1982|, |Sod, 1989|, |Chapra and Canale, 1990|, |'Thomas, 1995|, |Cuminato and Meneguette, 1999|, |Cunlia, 2000|, |Fortuna, 2000| e outros.

\subsection{Método de Diferenças Finitas Generalizadas uti- lizando Mínimos Quadrados}

O método DFG é desenvolvido a partir do método de aproximação por mínimos quadrados localizados (MIS) ${ }^{1}$, introdurido por Shepard, o qual fornece uma alternativa às interpolações clássicas de aproximação de funções a partir de seus valores dados em uma série de pontos distribuídos irregularmente. O MLS para a solução numórica de equaçòes dife-

\footnotetext{
'moving least square
} 
renciais tem recebido grande destaque, gerando uma série de métodos chamados meshless, os quais possibilitam uma aproximação numérica a partir de um conjunto de pontos que podem não ter como suporte uma malha o1 triangulação. Aqui ć utilizada a definição na qual um método é considerado meshless se as bases da aproximação são construídas a partir de um suporte arbitrário gerado por uma coleção de nós distribuídos irregularmente. A idéia de utilizar nós postos irregularmente num domínio para a obtenção das aproximações das diferenças finitas surgiu no final dos anos 60, Jensen |Jensen, 1972| apresenta. um método de DF que utiliza cólulas irregulares com seis pontos. Ltilizando a expansão em série de Taylor ele obtém uma formulação de DF que aproxima derivadas até de segunda ordem. A principal desvantagem deste método é que apresenta frequentes singularidades o11 mal condicionamento da célula. Perrone e Kao |Perrone and Kao, 1975| sugerem a adição de mais nós na célula e a aplicação de uma média para a geração dos coeficientes das DF. Liska e Orkisz |Liska and Orkisz, 1980| contribuíram com o desenvolvimento do método no q11e se refere à seleção de células na tentativa de eliminar os problemas indicados acima, aplicando o método na solução de problemas lineares e não lineares. Os trabalhos em |Luo and Haussler, 2002|, |Urena et al., 2001|, |Marshall and Grand, 1997|, o |Gossler, 2001| fazem 11so do método na construção de DF, expandindo suas aplicações para a solıção de diferentes problemas.

Os métodos meshless podem ser divididos cm duas catcgorias: os métodos baseados sob princípios variacionais e métodos que atuam diretamente nas equações diferenciais governantes. Os métodos da primeira categoria têm como característica comum a utilização de uma integração numérica para o estabelecimento das equações discretas do sistema. Na seginda categoria de métodos meshless é considerado o método de DFG, no qual um conjunto de equações discretas é estabelecido diretamente a partir das equações diferenciais. Fmbora o método de DFG scja um mótodo meshless, é interessante utilizar malhas (estruturadas ou não estruturadas) como suporte dos nós, com a q1al pode-se garantir uma melhor distribuição dos nós sobre o domínio obtendo uma rápida busca de vizinhança.

\subsubsection{Célula Computacional associada a um Nó}

Seja um domínio $D \subset \mathbb{R}^{d}$ e um conjunto de nós $V \quad\left\{v_{1}, \ldots, v_{n_{v}}\right\}$ tais que $V \subset D$. A cólula computacional ou simplesmente célula do nó $v_{i} \in V$, denotada $C_{i}$, é definida como o conjunto de $n_{i}$ nós $v_{k} \in V$, que são utilizadas no cálculo dos valores aproximados das derivadas de uma função contínua no nó $v_{i}$. As células dos nós são também conhecidas na literatura como estrelas de nó ou moléculas computacionais quando é incluído o nó principal.

Quando a discretização do domínio não pode ser regular devido a fatores como uma geometria complexa do domínio o1 refinamento local da malha, se faz necessário utilizar outros métodos de aproximação, como o método de DFG que apresentarmos aqui, nos quais a busca e escolha dos nós que compõom uma célula não é tão imediata como no caso regular.

O critério para a escolha dos nós de uma célula emprega como suporte uma malha não estruturada formada por elementos triangulares num domínio bidimensional (tetraedros, se o domínio é tridimensional), assim, as relações de vizinhança podem ser obtidas rapidamente utilizando uma estrutura de dados apropriada. Para nós identificados pelos vértices da malha utilizaremos três tipos de células: 
- Célula C(I): Neste tipo de cólula, os nós que compõem a célula de 11 mó $v_{i}$, correspondem aos vértices que compartilham uma aresta com $v_{i}$, tal como pode ser observado na figura (1.1a). () métdo DFG precisa de uma quantidade mínima de nós, e podem existir cólulas com uma quantidade de nós inferior ao exigido pelo método, assim na implementação, deve-se ter muito cuidado com este tipo de célıla. Geralmente, este problema ocorre para células $\mathrm{C}(\mathrm{I})$ de nós no bordo do domínio. Este tipo do cólula é a que gera menos erros ao implementar o método DFG.

- Célula C(II): Para $11 m$ nó $v_{i}$, este tipo de célula é composto pelos nós da célula C(I) mais os vértices opostos a $v_{i}$ por arestas cujos extremos estão em C(I), como apresenta a. figura (1.1b). Por possuir mais nós, $\mathrm{C}(\mathrm{II})$ evita o problema de possuir um número insuficiente de nós para o método DFG, mas apresenta a desvantagem de gerar maiores erros de aproximação.

- Célula C(III): Fste tipo de célula é composto pelos nós da célula C(I) relacionada á $v_{i}$, mais os vértices que compartilham uma aresta como os nós em $\mathrm{C}(\mathrm{I})$, com exceção de $v_{i}$. A figura (1.1c) apresenta um exemplo deste tipo de célula. Estas células scrão utilizadas unicamente em casos excepcionais, onde células $\mathrm{C}$ (II) não possuam quantidade mínima de nós exigida pelo método de: DFG. Um caso típico onde se utiliza estas cóllulas são nos nós que estão nos "cantos" das malhas.

Para células onde os nós devem ser posicionados no baricentro dos elementos triangulares será utilizado uma célula tipo C(IV), definida como:

- Célula(IV): Os nós que compõem a célula tipo C(IV) pará um nó $b_{i}$ no baricentro de um elemento triangular, são dados pelos nós que estão nos elementos triangulares que compartilham vértices olı arestas com o triângulo do nó $b_{i}$. A figura (1.1d) apresenta $11 \mathrm{~m}$ exemplo deste tipo de célula.

Para cada nó $v_{i} \in V$ sitıado na posição $\mathbf{r}_{i}=\left(x_{i}^{1}, \ldots, x_{i}^{d}\right)^{2}$ pode-se definir um novo sistema de coordenadas cuja origem está sitıada em $\mathbf{r}_{i}$, com o qual, uma posição qualquer $\mathbf{r}=\left(x^{1}, \ldots, x^{d}\right)$ é expressa neste novo sistema de coordenadas como $\overline{\mathbf{r}}=\left(\bar{x}^{1}, \ldots, \bar{x}^{d}\right)$ com seus componentes dados por:

$$
\bar{x}^{j}=x^{j}-x_{i}^{j} \quad \text { para } \quad j=1, \ldots, d .
$$

assim, as posições dos nós $v_{k} \in C_{i}$, podem ser expressas como $\overline{\mathbf{r}}_{k}=\left(\bar{x}_{k}^{1}, \ldots, \bar{x}_{k}^{d}\right)$.

Como uma medida de tamanho, para cada cólula $C_{i}$, se define o raio da cólula $\rho_{i}$, como $\rho_{i}=\min _{k \in C_{i}} \rho_{i, k}$, onde $\rho_{i, k}$ são as distâncias eluclidianas de cada um dos nós $v_{k}$ da cóluláa com o nó principal $v_{i}$, assim $\rho_{i, k} \cdots\left\|\tilde{\mathbf{r}}_{k}\right\|_{2}$ (comprimento da aresta $v_{i} v_{k}$ ). Portanto, pode-se introduzir um parâmetro de comprimento global da malha $h$ como:

$$
h=\min _{v_{i} \in V} \rho_{i},
$$

que é simplesmente igual ao comprimento da menor aresta da malha.

\footnotetext{
${ }^{2} x_{i}^{j}-\left\langle v_{i}, e_{j}\right\rangle$, onde $e_{j}$ são os elementos da basc canônica.
} 


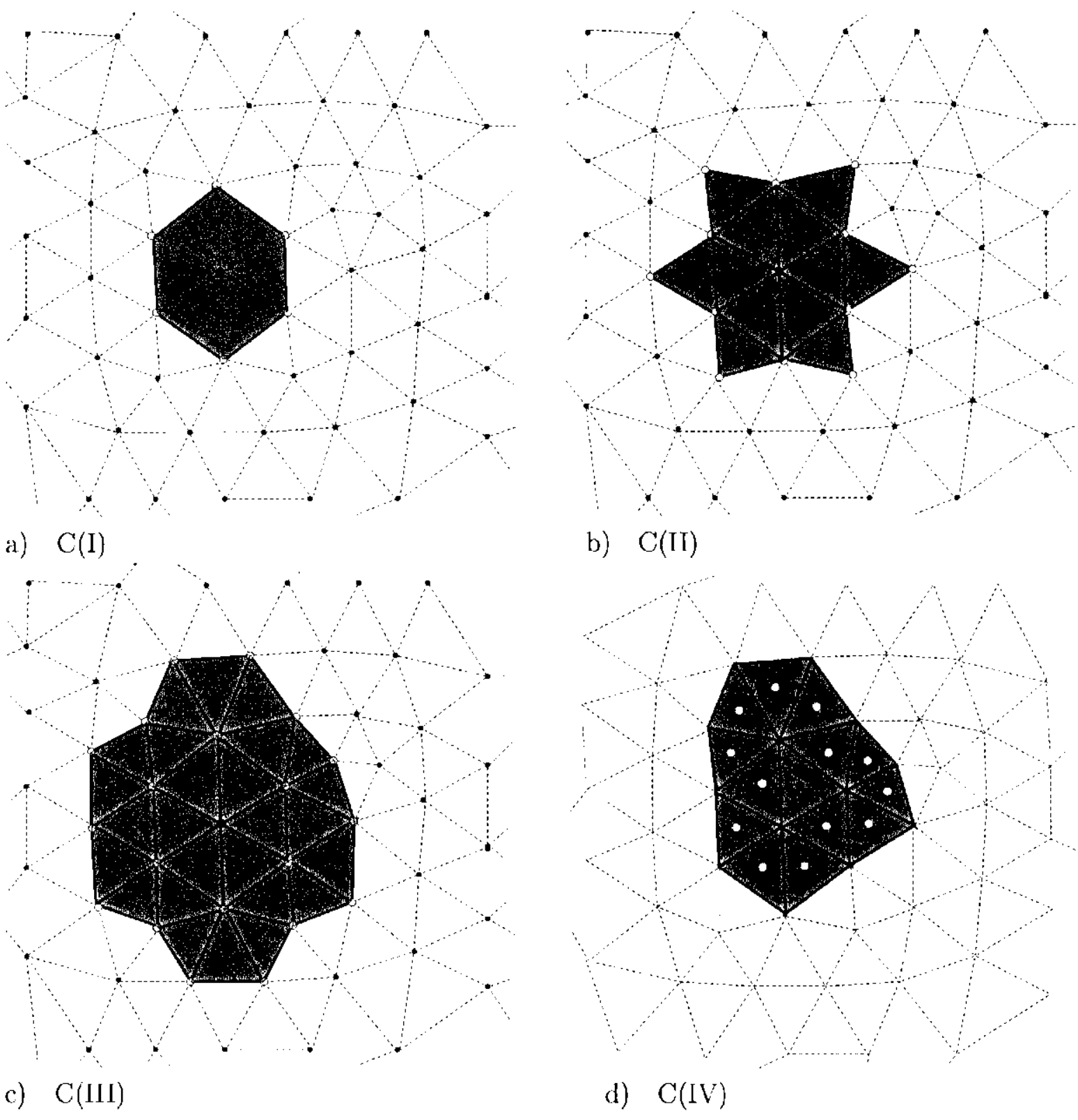

Figura 1.1: Diferentes tipos de célula de nó.

\subsubsection{Aproximação por Mínimos Quadrados}

Seja $f: D \longrightarrow \mathbb{R}$ uma função de classe $C^{q}$ definida $\mathrm{em} D \subset \mathbb{R}^{d}$ e um conjunto de nós $V \subset D$. Suponha que para qualquer nó $v_{0} \in V$ é definida uma célula $C_{0}$, e o valor de $f\left(\mathbf{r}_{0}\right)$ ć conhecido.

Procura-se uma função $\bar{f}_{0}$ que aproxime $f$ na vizinhança de $v_{0}$ e cujas derivadas de ordem menor ou ignal a $s<q$ sejam fáceis de calcular. Lma boa altornativa é utilizar um polinômio de ordem $s>0$ ajustado por mínimos quadrados com os valores da função $f$ nos nós de $C_{0}$. Suponha que

$$
\bar{f}_{0}(\overline{\mathbf{r}})=f\left(\mathbf{r}_{0}\right)+W_{0}(\mathbf{r})
$$


onde $W_{0}$ é um polinômio de grau $s$ dado por

$$
W_{0}(\overline{\mathbf{r}})=\sum_{j=1} c_{j} P_{0}^{(j)}(\mathbf{r})
$$

onde $P_{0}^{(j)}(\overline{\mathbf{r}})$ expressam os clementos de uma base do espaço polinomial $\mathcal{P}_{s}^{(d)}$ e $c_{j}$ sâo os coeficientes correspondentes. O polinômio de aproximaşão $W_{0}(\tilde{\mathbf{r}})$ deve ser nulo sob o nó $v_{0}$. Cada um dos elementos $P_{0}^{(j)}(\overline{\mathbf{r}})$ da base polinomial é um monômio de grau menor ou igual a $s$, com exceção do monômio de grau 0 , que pode ser desconsiderado ${ }^{3}$. Por simplicidade, identificaremos $P_{0}^{(j)}(\overline{\mathbf{r}})$ como $P^{j}, W_{0}(\overline{\mathbf{r}})$ como $W_{0}$, e $P_{0}^{(j)}\left(\mathbf{r}_{k}\right)$ como $P_{k}^{(j)}$ quando $\left(\mathbf{r}_{k}\right)$ é o vetor posição do nó $v_{k}$, deixado implícito que estes elementos estão relacionados ao nó $v_{0}$.

Exemplo 2.1. Ao utilizar um polinômio de primeiro grau $(s=1)$ numa função de aproximação $\bar{f}_{0}$, para um problema umidimensional, $W_{0}$ será dado como: $W_{0}(\bar{x})=c_{1} P^{1}=$ $c_{1} \bar{x}$ assim, $f$ é aproximado no ponto $x_{0}$ por $\bar{f}_{0}$ que será uma linha reta em $\mathbb{R}^{2}$. Para um problema bidimensional, $W_{0}$ será dado por

$$
W_{0}(\bar{x}, \bar{y})=c_{1} P^{1}+c_{2} P^{2}=c_{1} \bar{x}+c_{2} \bar{y}
$$

com o qual a função de aproximação $f_{0}$ ć um plano em $\mathbb{R}^{3}$. Os coeficientes $c_{i}$ do polinômio $W_{0}$ aproximam as derivadas de $f$ sob o nó $v_{0}$. No seguinte exemplo pode ser apreciado este fato.

Exemplo 2.2. Suponha uma função $f: D \subset \mathbb{R}^{2} \longrightarrow \mathbb{R}$ a qual é aproximada por $\bar{f}_{0}$, sobre o nó $v_{0}$ a partir de um polinômio de aproximação

$$
W_{0}(\bar{x}, \bar{y})=c_{1} \bar{x}+c_{2} \bar{y}+c_{3} \bar{x}^{2}+c_{4} \bar{x} \bar{y}+c_{5} \bar{y}^{2} .
$$

As derivadas $\frac{\partial f}{\partial x}, \frac{\partial f}{\partial y}, \frac{\partial^{2} f}{\partial x^{2}}, \frac{\partial^{2} f}{\partial x \partial y}, \frac{\partial^{2} f}{\partial y^{2}}$ podem ser estimadas de $\frac{\partial f_{0}}{\partial x}, \frac{\partial \bar{f}_{0}}{\partial y}, \frac{\partial^{2} f_{0}}{\partial x^{2}}, \frac{\partial^{2} \bar{\jmath}_{0}}{\partial x \partial y}, \frac{\partial^{2} \bar{f}_{0}}{\partial y^{2}}$, devido a que $\overline{\mathbf{r}}_{0}=(0,0)$ de $(1.2)$ obtém-se:

$$
\begin{aligned}
\frac{\partial \bar{f}_{0}}{\partial \bar{x}} & =\frac{\partial W_{0}}{\partial \bar{x}}=c_{1}+2 c_{3} \bar{x}+c_{4} \bar{y}=c_{1} \\
\frac{\partial \bar{f}_{0}}{\partial \bar{y}} & =\frac{\partial W_{0}}{\partial \bar{y}}=c_{2}+c_{4} \bar{x}+2 c_{5} \bar{y}=c_{2} \\
\frac{\partial^{2} \tilde{f}_{0}}{\partial x^{2}} & =\frac{\partial^{2} W_{0}}{\partial \bar{x}^{2}}=2 c_{3} \\
\frac{\partial^{2} \bar{f}_{0}}{\partial \bar{x} \partial \bar{y}} & =\frac{\partial^{2} W_{0}}{\partial \bar{x} \partial y}=c_{4} \\
\frac{\partial^{2} \tilde{f}_{0}}{\partial y^{2}} & =\frac{\partial^{2} W_{0}}{\partial \bar{y}^{2}}-2 c_{5} .
\end{aligned}
$$

\footnotetext{
${ }^{3}$ Por definição, o espaço polinomial $\mathcal{P}_{s}^{(d)}$ é composto por todos os polinómios d-dimensionais de grau menor om igual a $s$, incluindo os monomios de grau zero. Neste trabalho o espaço $\mathcal{P}_{s}^{(d)}$ corresponde ao conjunto de todos os polinomios d-dimensionais de grau $s^{\prime}$, onde $0<s^{\prime} \leq s$. Em problemas de interpolação, o espaço $\mathcal{P}_{s}^{(d)}$ deve incluir os polinômios de grau zero.
} 
Cada problema necessitia de uma base $P^{(1)}, \ldots, P^{(n)}$ para formar o espaço polinomial $\mathcal{P}_{s}^{d}$ (tal como se observa nos exemplos 2.1 e 2.2 ). Cada um dos elementos da base $P^{(j)} \dot{c}$ definido como um monomio de graı $s^{\prime}$, com $0<s^{\prime} \leq s$, que é expresso como

$$
P^{(j)} \cdots\left(\bar{x}^{1}\right)^{\mu_{1}(j)}\left(\bar{x}^{2}\right)^{\mu_{2}(j)} \cdots\left(\bar{x}^{d}\right)^{\mu_{d}(j)},
$$

onde $\mu_{k}(j)$ é um númcro inteiro, com $k-1, \ldots, d$, satisfazendo as seguintes restrições

$$
0 \leq \mu_{k}(j) \leq s^{\prime} \quad \text { ○ } \quad \sum_{k=1}^{d} \mu_{k}(j)=s^{\prime} .
$$

A base do espaço polinomial $\mathcal{P}_{s}^{(d)}$ ó composta pelos elementos da base que possuem os monômios de grau 1 , em seguida os monômios de grau 2 e assim até completar todos os monômios de gran $s$, tal como é apresentado na tabela 1.2 .2 , para os espaços polinomiais $\mathcal{P}_{3}^{(1)}, \mathcal{P}_{3}^{(2)}$ с $\mathcal{P}_{2}^{(3)}$

a)

\begin{tabular}{|c|c|c|}
\hline \multicolumn{3}{|c|}{$\mathrm{d}=1$} \\
\hline monômio & $\mu_{1}$ & gralı \\
\hline$P^{(1)}=\bar{x}$ & 1 & 1 \\
\hline$P^{(2)}=\bar{x}^{2}$ & 2 & 2 \\
\hline$P^{(3)}=\bar{x}^{3}$ & 3 & 3 \\
\hline
\end{tabular}

b)

\begin{tabular}{|c|c|c|c|}
\hline \multicolumn{5}{|c|}{$\mathrm{d}=2$} \\
\hline monomio & $\mu_{1}$ & $\mu_{2}$ & graul \\
\hline$P^{(1)}=x$ & 1 & 0 & 1 \\
$P^{(2)}=y$ & 0 & 1 & 1 \\
\hline$P^{(3)} \bar{x}^{2}$ & 2 & 0 & 2 \\
$P^{(4)}=\bar{x} \bar{y}$ & 1 & 1 & 2 \\
$P^{(5)}=\bar{y}^{2}$ & 0 & 2 & 2 \\
\hline$P^{(6)}=\bar{x}^{3}$ & 3 & 0 & 3 \\
$P^{(7)}=\bar{x}^{2} y$ & 2 & 1 & 3 \\
$P^{(8)}=\bar{x} \bar{y}^{2}$ & 1 & 2 & 3 \\
$P^{(9)}=\bar{y}^{3}$ & 0 & 3 & 3 \\
\hline
\end{tabular}

c)

\begin{tabular}{|c|c|c|c|c|}
\hline \multicolumn{5}{|c|}{$\mathrm{d}=3$} \\
\hline monômio & $\mu_{1}$ & $\mu_{2}$ & $\mu_{3}$ & graul \\
\hline$P^{(1)}=\bar{x}$ & 1 & 0 & 0 & 1 \\
$P^{(2)}=\bar{y}$ & 0 & 1 & 0 & 1 \\
$P^{(3)} \ldots \bar{z}$ & 0 & 0 & 1 & 1 \\
\hline$P^{(4)}=\bar{x}^{2}$ & 2 & 0 & 0 & 2 \\
$P^{(5)}=x y$ & 1 & 1 & 0 & 2 \\
$P^{(6)}=\bar{x} \bar{z}$ & 1 & 0 & 1 & 2 \\
$P^{(7)}=\bar{y}^{2}$ & 0 & 2 & 0 & 2 \\
$P^{(8)}-\bar{y} \bar{z}$ & 0 & 1 & 1 & 2 \\
$P^{(9)}=\bar{z}^{2}$ & 0 & 0 & 2 & 2 \\
\hline
\end{tabular}

Tabela 2.2 .2 :Bases dos espaços polinomiais a) $\mathcal{P}_{3}^{(1)}$, b) $\mathcal{P}_{3}^{(2)}$ e c) $\mathcal{P}_{2}^{(3)}$.

Conhecendo os monômios $P^{(j)}$ o problema de calcular $W_{0}$ se resume em obter os coeficientes $c_{1}, \ldots, c_{n}$, que aproximam a função $\bar{f}_{0}$ nà região da cólula $C_{0}$. Utilizando para isto um ajuste de mínimos quadrados, calculam-se tais coeficientes; obtendo o segninte sistcma lincar, onde os coeficientes $c_{i}$ estarão contidos no vetor das incógnitas

$$
\mathrm{A} \mathrm{c}=\mathrm{b}
$$

onde A é a matriz composta pelos elementos $a_{i, j}$ dados por,

$$
a_{i, j}-\sum_{v_{k} \in C_{0}^{j}} P_{k}^{(i)} P_{k}^{(j)} \omega_{k} \quad \text { com } \quad i, j=1, \ldots, n,
$$




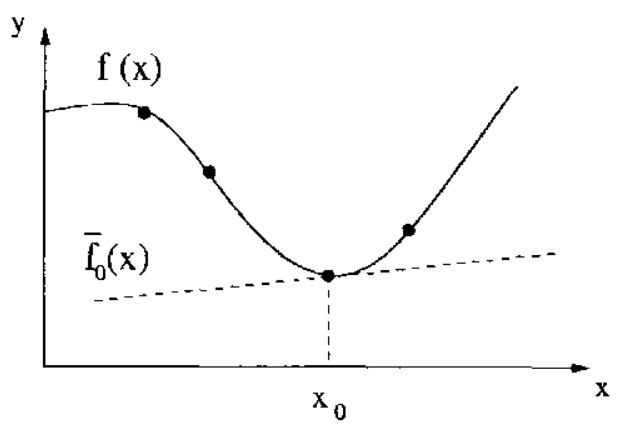

(a)

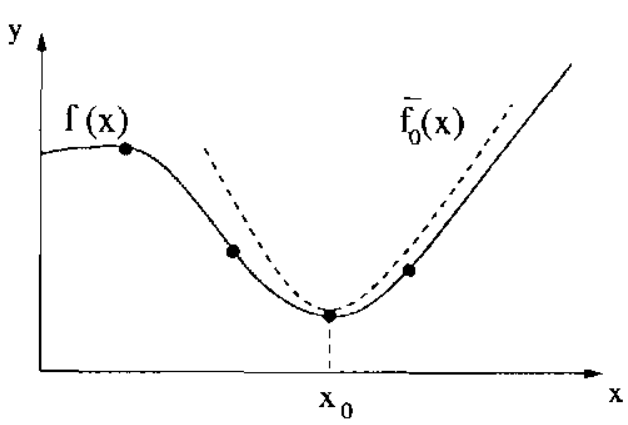

(b)

Figura 1.2: Função de aproximação $f_{0}$ : (a)uma linha reta $\mathrm{com} s=1$, e (b) uma parábola $\operatorname{com} s=2$.

o vetor de incógnitas c $\quad\left[c_{1}, \ldots, c_{n}\right]^{T}$ e $\mathbf{b}=\left[b_{1}, \ldots, b_{n}\right]^{T}$ onde $b_{i}$ denotam os produtos escalares

$$
b_{i}=\sum_{v_{k} \subset C_{0}}\left(f\left(\mathbf{r}_{k}\right)-f\left(\mathbf{r}_{0}\right)\right) P_{k}^{(i)} \omega_{k}
$$

avaliados sobre os nós $v_{k} \in C_{0}$. A função peso $\omega_{k}$ foi introdurida, e geralmente, depende das posições entre os nós $v_{0} \mathrm{e} v_{k}$. Algumas vezes, a função peso $\omega_{k}$ ć utilizada para privilegiar nós numa dada direção. Neste trabalho utilizamos funções peso isotrópicas, onde o valor depende unicamente da distancia de um ponto qualquer a.o nó vo:

$$
\omega_{k}-\omega_{k}\left(\rho_{0, k}\right)
$$

A função peso não deve incremontar-se com seu argumento, isto é,

$$
\omega_{a} \geq \omega_{b} \Longleftrightarrow \rho_{0, a} \leq \rho_{0, b},
$$

onde $v_{a}, v_{t} \in V$, assim, os nós mais distantes do nó central $v_{0}$, tềm menos influência na aproximação que os nós mais próximos a $v_{0}$. Ta formulação de aproximação por mínimos quadrados, é assumido que a função peso tem um suporte limitado a uma bola finita de raio $R$

$$
\omega_{a}\left(\rho_{0, a}\right)=0 \Longleftrightarrow \rho_{0, a}>R
$$

Para evitar que o sistema (1.6) seja indeterminado, é importante que qualquer célula computacional $C_{0}$, tenha uma quantidade de nós igual ou maior a $n$ (número de monômios).

Para o estudo da consistência das aproximações das derivadas obtidas pelo método DFG, analisamos a solução do sistema (1.6).

Seja um nó qualquer $v_{0} \in V$. Supondo que o polinômio de aproximação $W_{0} \in \mathcal{P}_{s}^{(d)}$, a função $\bar{f}$ ć dada por (1.2) e a matriz A do sistema linear (1.6) utilizada no ajuste por mínimos quadrados de $\bar{f}$ não é singular, então, seu determinante pode ser expresso mediante a regra de Laplace como,

$$
\operatorname{det}(\mathrm{A})=\sum_{j=1}^{n}(-1)^{(i+j)} a_{i, j} \operatorname{det}\left(A_{i, j}\right)
$$

onde $A_{i, j}$ é a matriz de ordem $n-1$ obtida ao climinar a i-śsima coluna e a j-ésima linha da matriz A. Aplicando a regra de Cramer para solucionar o sistema (1.6), cada um dos 
coeficientes $c_{i}$ do vetor c, será dado por

$$
c_{i}=\sum_{j=1}^{n}(-1)^{(i+j)} b_{j} S_{j}^{(i)}
$$

onde o elemento $S_{j}^{(i)}$ é

$$
S_{j}^{(i)}=\frac{\operatorname{det}\left(\mathbf{A}_{i, j}\right)}{\operatorname{det}(\mathbf{A})} .
$$

Utilizando a definição dos elementos $b_{i}$ dada em (1.8) no sistema e coordenadas própria do nó $v_{0}$, c substituindo cm (1.13), obtém-se

$$
c_{i} \cdots \sum_{j=1}^{n} \sum_{u_{k} \in C_{0}}\left[f\left(\mathbf{r}_{k}\right)-f\left(\mathbf{r}_{0}\right)\right] P_{k}^{(j)} S_{j}^{(i)} \omega_{k},
$$

onde a funçăo $f$ avaliada em qualquer nó $v_{k}$ é equivalente a:

$$
f\left(\mathbf{r}_{k}\right)=f\left(x_{0}^{1}+x_{k}^{1}, x_{0}^{2}+\bar{x}_{k}^{2}, \ldots, x_{0}^{d}+x_{k}^{d}\right)=f\left(x_{0}^{1}+P_{k}^{(1)}, x_{0}^{2}+P_{k}^{(2)}, \ldots, x_{0}^{d}+P_{k}^{(d)}\right),
$$

assim, ao expandir por série de Taylor a função $f$ ao redor do $\mathbf{r}_{0}$, obtém-se

$$
f\left(\mathbf{r}_{k}\right)=\sum_{l^{\prime}=0}^{\infty} \frac{1}{l^{\prime \prime}}\left(\sum_{m=1}^{d} P_{k}^{(m)} \frac{\partial}{\partial x^{m}}\right)^{l^{\prime}} f\left(\mathbf{r}_{0}\right)
$$

E pode ser expressa por

$$
f\left(\mathbf{r}_{k}\right)=f\left(\mathbf{r}_{0}\right)+\sum_{l=1}^{\infty} \frac{1}{\pi(l)} \prod_{m=1}^{d}\left(P_{k}^{(m)} \frac{\partial}{\partial x^{m}}\right)^{\mu_{m}(l)} f\left(\mathbf{r}_{0}\right),
$$

$\operatorname{com} \pi(l)=\prod_{m=1}^{d} \mu_{m}(l)$ !. F o produtório pode ser expresso por

$$
\prod_{m=1}^{d}\left(P_{k}^{(m)} \frac{\partial}{\partial x^{m}}\right)^{\mu_{m}(l)}=\prod_{m=1}^{d}\left(P_{k}^{(m)}\right) \prod_{m=1}^{d}\left(\frac{\partial}{\partial x^{m}}\right)^{\mu_{m}(l)}
$$

lembrando que $P_{k}^{(m)}=\bar{x}_{k}^{m n}$ para $m=1, \ldots, d$, utilizando a definição de $P^{(j)}$ dada $\mathrm{cm}(1.4)$

$$
\prod_{m-1}^{d}\left(P_{k}^{(m)}\right)_{m l}^{\mu} \quad\left(\bar{x}_{k}^{1}\right)^{\mu_{1}(l)} \cdots\left(\bar{x}_{k}^{d}\right)^{\mu_{d}(l)}=P_{k}^{(l)}
$$

e definindo o operador diferencial $D_{l}$

$$
D_{l}=\prod_{m=1}^{d}\left(\frac{\partial}{\partial x^{m}}\right)^{\mu_{m}(l)}=\frac{\partial^{N_{d}(l)}}{\left(\partial x^{1}\right)^{\mu_{1}(l)} \cdots\left(\partial x^{d}\right)^{\mu_{d}(l)}},
$$

pode-se escrever (1.17) como

$$
f\left(\mathbf{r}_{k}\right)=f\left(\mathbf{r}_{0}\right)+\sum_{l=1}^{\infty}\left(\frac{1}{\pi(l)} P_{k}^{(l)} D_{l}\right) f\left(\mathbf{r}_{0}\right) .
$$


Substituindo (1.19) na equação (1.24) obtém-se

$$
c_{i}=\sum_{j=1}^{n} \sum_{v_{k} \in C_{0}} \sum_{l=1}^{\infty} \frac{1}{\pi(l)} P_{k}^{(l)} P_{k}^{(j)} S_{j}^{(i)} \omega_{k}\left[D_{l} f\left(\mathbf{r}_{0}\right)\right],
$$

separando $\mathrm{cm}(1.20)$ os termos com parâmetro $l$ maiores que $n$ e fatorando os termos que são menores ou iguais a $n$, obtemos

$$
\begin{aligned}
c_{i}= & \sum_{j=1}^{n} \sum_{v_{k} \subset C_{0}}\left(\sum_{l=1}^{n} \frac{1}{\pi(l)} P_{k}^{(l)} P_{k}^{(j)} S_{j}^{(i)} \omega_{k}\left[D_{l} f\left(\mathbf{r}_{0}\right)\right]\right) \\
+ & \sum_{j=1}^{n} \sum_{v_{k} \subset C_{1}}\left(\sum_{l=n \nmid 1}^{\infty} \frac{1}{\pi(l)} P_{k}^{(l)} P_{k}^{(j)} S_{j}^{(i)} \omega_{k}\left[D_{l} f\left(\mathbf{r}_{0}\right)\right]\right) \\
& \sum_{l=1}^{n}\left(\frac{1}{\pi(l)}\left[D_{l} f\left(\mathbf{r}_{0}\right)\right] \sum_{j=1}^{n}\left(S_{j}^{(i)} \sum_{v_{k} \in C_{0}} P_{k}^{(l)} P_{k}^{(j)} \omega_{k}\right)\right)+\bar{T}_{i, n},
\end{aligned}
$$

onde o termo $\tilde{T}_{i, n}$, contém os elementos de ordem superior $(l>n)$. Substituindo (1.7) na expressiano (1.21) tem-se

$$
c_{i}=\sum_{l-1}^{n}\left(\frac{1}{\pi(l)}\left[D_{l} f\left(\mathbf{r}_{0}\right)\right] \sum_{j=1}^{n} S_{j}^{(i)} a_{j, l}\right)+\bar{T}_{i, n},
$$

onde $a_{j, l}$ são os elementos da matriz, A.

Seja

$$
\sum_{j=1}^{n} S_{j}^{(i)} a_{j, l} \cdots \delta_{i, l}, \operatorname{com} i, l \quad 1, \ldots, n,
$$

onde $\delta_{i, l} \dot{c}$ a função deltia de $\operatorname{Kronecker}\left(\delta_{i, l}=1 \quad\right.$ se $i=l$ e $\quad \delta_{i, l}=0$ se $\left.i \neq l\right)$.

Aplicando (1.23) na equação (1.22), obtém-se

$$
\left.c_{i}=\sum_{l=1}^{n}\left(\frac{1}{\pi(l)} \mid D_{l} f\left(\mathbf{r}_{0}\right)\right] \delta_{i, l}\right)+\bar{T}_{i, n}=\frac{1}{\pi(i)}\left[D_{i} f\left(\mathbf{r}_{0}\right)\right]+\ddot{T}_{i, n}
$$

Portanto, o coeficiente $c_{i}$ do polinômio de aproximação $W_{0}$ é igual ao valor da derivada. $D_{i}$ da função $f$ no ponto $\mathbf{r}_{0}$, mais um termo extra $T_{i, n}$, que é formado por clementos de ordem superior, e dele depende o erro de truncamento do método. Por definição, o termo $\bar{T}_{i, n}$ é dado como:

$$
\dddot{T}_{i, n}=\sum_{l=1}^{\infty} T_{i, n \cdots l}
$$

onde

$$
T_{i, n+l} \cdots \frac{1}{\pi(n+l)}\left[D_{(n+l)} f\left(\mathbf{r}_{0}\right)\right] \sum_{j=1}^{n_{1}} \sum_{v_{k} \subset C_{i}} P_{k}^{(n+l)} P_{k}^{(j)} S_{j}^{(i)} \omega_{k}
$$

Para que $c_{i}$ seja uma aproximação consistente, devemos utilizar urm polinômio de aproximação $W_{0}$ que utiliza todos os monômios da espaço $\mathcal{P}_{s}^{(d)}$. Detalhes pode ser encontrado em |Реña, 2003|. 


\subsubsection{Implementação das Equações}

$\Lambda$ implementação do método de DFG é constituída pelos seguintes passos:

- Análise da EDP a ser solucionada e a escolha dos espaços dos polinômios de aproximaça mais convenientes;

- Criação de uma boa distribuição de nós sob o domínio (geração de uma malha) e escolha das células apropriadas para cada nó;

- Obtenção das aproximações e solıção dos sistemas de equações discretas.

Considerando problemas bidimensionais, as EDPs serão equações envolvendo duas variáveis independentes $x$ e $y$ c derivadas parciais de uma função real $f: \ldots f(x, y)$. A forma mais geral de uma EDP bidimensional de ordem $s$ é :

$$
\phi\left(x, y, f, \frac{\partial f}{\partial x}, \frac{\partial f}{\partial y}, \frac{\partial^{2} f}{\partial x^{2}}, \frac{\partial^{2} f}{\partial x \partial y}, \frac{\partial^{2} f}{\partial y^{2}}, \frac{\partial^{s} f}{\partial x^{s}}\right)=0 .
$$

É bastante comum o caso de problemas práticos importantes onde a equação diferencial parcial é de ordem $s \ldots 2$ e linear nas derivadas de ordem 2, portanto

$$
a \frac{\partial^{2} f}{\partial x^{2}}+b \frac{\partial^{2} f}{\partial x \partial y}+c \frac{\partial^{2} f}{\partial y^{2}} \cdots \phi\left(x, y, f, \frac{\partial f}{\partial x} \frac{\partial f}{\partial y}\right)
$$

Ao solucionar numericamente uma equação como (1.28), o domínio D deve inicialmente ser representado por um conjunto de nós $V$, sobre tais pontos utilizamos o método de DFG para discretizar as EDPs, as quais são solucionadas utilizando as condições de fronteira e as condiçoss iniciais.

Para cada nó $v_{0} \in V$, devemos procurar um polinômio de aproximação $W_{0}$ a partir do qual, possamos obter os elementos $c_{i}$ associados às derivadas $D_{i}$. Uma boa opção é utilizar um polinômio $W_{0}$ que pertença ao espaço polinomial $P_{s^{\prime}}^{2}$, sendo $s^{\prime} \geq s=2$. Por exemplo, considere os polinômios $W_{0}^{2} \in P_{2}^{2}$ e $W_{0}^{3} \in P_{3}^{2}$. No primeiro caso, ao utilizar $W_{0}^{2}$, teremos de (1.26) que a ordem do crro de truncamento das aproximações das derivadas de primeira e segunda ordens são $O(h)$ e $O\left(h^{2}\right)$ respectivamente, enquanto ao utilizar $W_{0}^{3}$, a ordem do erro de truncamento das derivadas de primeira e segunda ordens serão de $O\left(h^{2}\right)$ e $O\left(h^{3}\right)$, respectivamente. Portanto as aproximações das derivadas utilizando o polinômio de aproximação $W_{0}^{3}$ são melhores que as obtidas pelo polinômio $W_{0}^{2} . \Lambda$ ordem de complexidade do método de DFG é $O\left(n^{3} n_{v}\right)$, onde $n_{v}$ é o número total de nós do conjunto $V$, e $n$ é a dimensão do espaço polinomial $\mathcal{P}_{s}^{(d)}$. Tal complexidade é fácil de ser demonstrada pois devemos solucionar para cada nó de $V$, o sistema linear dado por (1.6), para o qual pode ser utilizado um método como o LU que tem ordem $O\left(n^{3}\right)$. Assim, a complexidade do método ao utilizar os polinômios de aproximaçño $W_{0}^{2}(\operatorname{com} n=5)$ e $W_{0}^{3}(\operatorname{com} n=9)$ em cada um dos nós de $V$ são $O\left(125 n_{v}\right)$ e $O\left(729 n_{v}\right)$ respectivamente; ol seja, o método terá um custo computacional quase seis vezes maior ao utilizar o polinômio de aproximação $W_{0}^{3}$. Portanto a escolha do espaço dos polinômios de aproximação são sujcitas aos critérios de aproximação da solução versus custo computacional. 


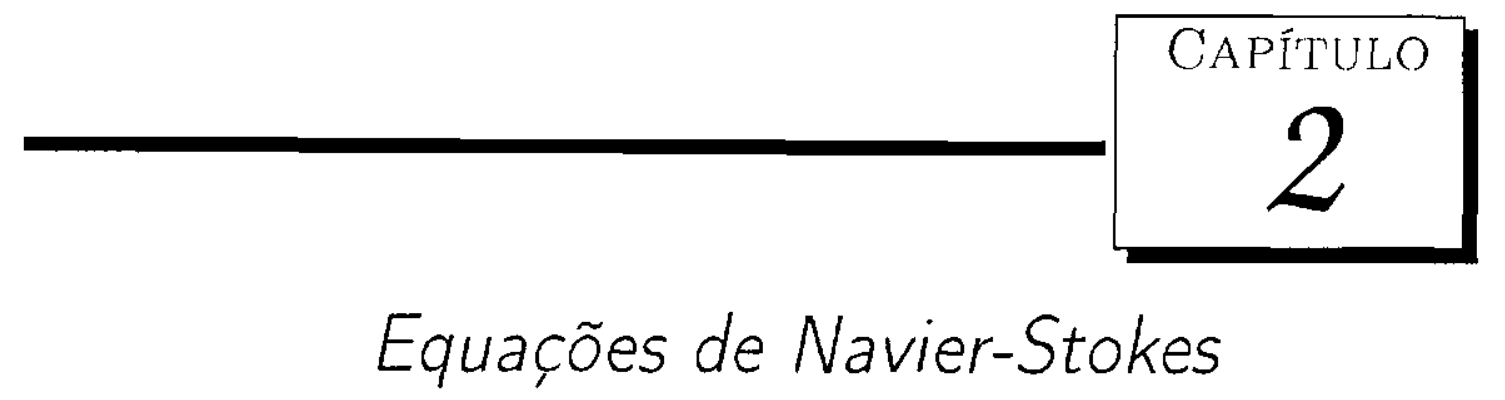

\subsection{Introdução}

No estudo dos escoamentos de fluidos incompressíveis, a modelagem matemática das leis de conservação é bem estabelecida pelas equações de conservação de massa e de Naviẹr-Stokes. A dinâmica de fluidos é a ciência que descreve o movimento de fluidos e estuda os ferômenos que envolvem Hluidos em movimento.

As leis da dinâmica dos fluidos são conhecidas como leis de conservação, para conhecer o cstado de $11 \mathrm{~m}$ fluido, deve-se determinar o valor das variáveis que o identificam, ao longo do tempo em cada ponto do espaço ocupado pelo fluido. As variáveis que identificam o estado de um fluido incompressívele isotêrmico são: a velocidade u em cada ponto (que é uma grandeza vetorial) e a pressão $p$ (que $\mathrm{em}$ cada ponto é uma grandeza escalar).

Essas variáveis são agrupadas em equaçòes, que passaram a ser conhecidas em todo mundo como, as equaçōes de Navier-Stokes. Detalhes sobre as equações de Navier-Stokes, dinâmica dos fluidos, leis de conservação, mecânica do contínuo e outras propriedades do escoamento de fluidos podem ser encontrados em |Batchelor, 1970|, |Peyret and Taylor, 1983|, |Panton, 1984|, |Flecther, 1992|, |Maliska, 1995| e |Ferziger and P'eríc, 1999|.

Neste capítulo será apresentado as equações de interesse, condições de contorno e algumas propriedardes dos fluidos.

\subsection{Descrição do Movimento de Fluido}

As maneiras de se especificar o movimento de um fluido em uma dada região dos espaço são denominadas como

- Formulação Lagrangeana

Na formulação I agrangeana define-se uma região material formada por um conjunto de partículas de fluido, denominada de volume de controle Lagrangeana. Conforme as partículas se movimentam no escoamento, a região se deforma, e não há fluxo de massa através de sulas faces. Nesta formulação, as grandezas do escoamento são 
especificadas como função do tempo e da partícula de fluido.

- Formulação Fuleriana

$\mathrm{Na}$ formulação Euleriana define-se uma região fixa no espaço, que não sc deforma com relação ao tempo, onde o comportamento do fluido será estudado e há fluxo de massa pelas faces do volume de controle.

Neste capitulo, as cquações de conservação serão desenvolvidas 11tilizando-se uma formulação Euleriana, onde as propriedades macroscópicas do escoamento dependem das coordenadas espaciais e temporal, como por exemplo, velocidade $\mathbf{u}=\mathbf{u}(\mathbf{x}, t)$, massa específica $\rho-\rho(\mathbf{x}, t)$ e viscosidade $\mu=\mu(\mathbf{x}, t)$.

\subsubsection{Derivada Total}

Segundo |Aris, 1962|: o movimento de um fluido pode ser descrito por uma transformação. Supõe-se quco cm um dado instante de tempo, uma determinada partícula esteja em uma posição $\boldsymbol{\xi} \in \mathbb{R}^{m}$, e num tempo posterior, a mesma partícula se encontre na posição $\mathbf{x} \in \mathbb{R}^{m}$. $\Lambda$ ssim, pode-so admitir que $\mathbf{x}$ é uma função de $t$ e da posição inicial $\boldsymbol{\xi}$, isto é

$$
\mathbf{x}-\mathbf{x}(\boldsymbol{\xi}, t)
$$

As coordenadas iniciais $\boldsymbol{\xi}$ são coordenadas materiais (o11 Lagrangeanas) e as coordenadas $\mathrm{x}$ são as coordenadas espaciais (ou Euleriana). Assume-se também que o movimento do fluido é uma função contínua, de modo que (2.1) pode ser invertida para recuperar as coordenadas materiais da partícula.

$$
\boldsymbol{\xi}=\boldsymbol{\xi}(\mathbf{x}, t)
$$

A descrição Lagrangeana da variação de uma propriedade $\phi(\xi, t)$ pode ser vista na descrição Fuleriana $\phi(\mathbf{x}, t)$ da seguinte forma |Ferreira, 2001|:

$$
\Phi(t)-\phi(\mathbf{x}(\boldsymbol{\xi}, t), t)
$$

Associadas às descrições I agrangeana e Euleriana, existem duas derivadas a considerar: a derivada com respeito ao tempo mantendo-se $x$ constante; e a derivada com respeito ao tempo mantendo-se $\boldsymbol{\xi}$ constante. A segunda derivada é frequentemente utilizada em dinâmica de fluidos a ó conhecida como derivada total ${ }^{1}$ da propriedade $\phi$. Assim, derivando-se $\Phi(t)$ com relação ao tempo, e deixando $\boldsymbol{\xi}$ constante, tem-se

$$
\begin{aligned}
\frac{\partial \Phi}{\partial t}(l) & =\frac{\partial \phi}{\partial t}\left(x_{1}(\boldsymbol{\xi}, t), x_{2}(\boldsymbol{\xi}, t), \ldots, x_{m}(\boldsymbol{\xi}, t), t\right) \\
& =\frac{\partial \phi}{\partial x_{1}} \frac{\partial x_{1}}{\partial t}+\frac{\partial \phi}{\partial x_{2}} \frac{\partial x_{2}}{\partial t}+\cdots+\frac{\partial \phi}{\partial x_{m}} \frac{\partial x_{m}}{\partial t}+\frac{\partial \phi}{\partial t} .
\end{aligned}
$$

Sendo

$$
\frac{\partial x_{i}}{\partial t}(\boldsymbol{\xi}, t)=u_{i}, \quad i \cdots 1,2, \ldots, m
$$

\footnotetext{
${ }^{1}$ Também chamada de derivada material ou substancial.
} 
tem-se a derivada material de: $\phi(\mathbf{x}, t)$, denotada por $\frac{D \phi}{D t}$,

$$
\frac{D \Phi}{D t} \equiv u_{1} \frac{\partial \phi}{\partial x_{1}}+u_{2} \frac{\partial \phi}{\partial x_{2}}+\cdots+u_{m} \frac{\partial \phi}{\partial x_{m}}+\frac{\partial \phi}{\partial t}
$$

ou em notação vetorial

$$
\frac{D \phi}{D t}=\frac{\partial \phi}{\partial t}+(\mathbf{u} \cdot \nabla) \phi
$$

\subsection{Equações Governantes}

Nesta seção descrevemos as equações de conservação de intcresse para o presente trabalho. O fluido será considerado um contínuo de massa, onde as propriedades do escoamento são descritas em termos de grandezas macroscópicas como pressão, velocidade e massa específica (densidade).

\subsubsection{Equação de Conservação de Massa}

O princípio da conservação de massa diz que na ausência de fontes ou sorvedouros, toda massa que entra em um volume do controle deve sair e/ou se acumular neste mesmo volume. Fste princípio é regido pela equaşão

$$
\frac{D \rho}{D t}+(\rho \nabla) \cdot \mathbf{u} \cdot 0
$$

Como a massa específica de uma partícula material não se altera no tempo, para escoamentos incompressiviveis, tem-se

$$
\frac{D \rho}{D t}=0
$$

e substituindo em (2.8), vem

$$
\rho \nabla \cdot \mathbf{u}--0
$$

e como $\rho /: 0$, tem-se

$$
\nabla \cdot \mathbf{u}=0 .
$$

A equação (2.11) ó conhecida como condição de incompressibilidade e será mantida em todo domínio, isto é, todos os fluidos envolvidos na simulação obedecerão esta condição, e portanto, serão tomados como incompressiveis.

\subsubsection{Equação do Balanço de Quantidade de Movimento}

A equação de balanço de quantidade de movimento linear é obtida aplicando-se a segunda lei de Newton, assim, a taxa de variação temporal da quantidade de movimento de uma partícula é igual a resultante das forças que agem sobre essa partícula, que ma forma vetorial è dada por

$$
\frac{D \rho \mathbf{u}}{D t}=\nabla \cdot \boldsymbol{\sigma}+\rho \mathbf{g}
$$

onde $\boldsymbol{\sigma}$ representa o tensor de tensões totais de escoamento e $\mathbf{g}$ o campo gravitacional. Utilizando $1 \mathrm{~m}$ modelo Newtoniano, este tensor pode ser escrito (omo

$$
\boldsymbol{\sigma}=-p \mathbf{I}+\boldsymbol{\tau}
$$


onde $p=p(\mathbf{x}, t)$ representa o campo de pressão do escoamento, I é o tensor identidade e $\boldsymbol{\tau}$ é o tensor de tensóes viscosas dado por

$$
\boldsymbol{\tau}=2 \mu \mathrm{D}+\left(\lambda-\frac{2}{3} \mu\right)(\nabla \cdot \mathbf{u}) \mathbf{I}
$$

onde $\mu$ é o coeficiente de viscosidade dinâmica e $\lambda$ o coeficiente volumêtrico do fluido. Devido a condição de incompressibilidade (2.11), pode-se escrever

$$
\boldsymbol{\tau}=2 \mu \mathbf{D} \text {. }
$$

O tensor $\mathbf{D}$ é denominado tensor deformação, e segundo o modelo Newtoniano, é dado por

$$
\mathrm{D}=\frac{1}{2} \dot{\gamma}(\mathbf{u}) \cdot \frac{1}{2}\left(\nabla \mathbf{u}+\nabla \mathbf{u}^{T}\right)
$$

onde $\dot{\gamma}(\mathbf{u})$ é denominado de tensor laxa de deformação. Assim, utilizando o tensor de tensões Newtoniano, pode-se calcular o divergente do tensor de tensões totais

$$
\nabla \cdot \boldsymbol{\sigma}=\nabla \cdot\left[-p \mathbf{I}+\mu\left(\nabla \mathbf{u}+\nabla \mathbf{u}^{T}\right)\right]=-\nabla p+\nabla \cdot\left[\mu\left(\nabla \mathbf{u}+\nabla \mathbf{u}^{T}\right)\right] .
$$

Deste modo, a equação de conservação de quantidade de movimento pode ser expressa como

$$
\frac{D(\rho \mathbf{u})}{D t}=-\nabla p+\nabla \cdot\left[\mu\left(\nabla \mathbf{u}+\nabla \mathbf{u}^{T}\right)\right]+\rho \mathbf{g} .
$$

\subsection{Equações Governantes na Forma Bidimensional}

Para discretizar as equações vistas até aqui na forma vetorial, é necessário escolher um sistema de coordenadas. Neste trabalho, as equações de conservação serão desenvolvidas no sistema cartesiano bidimensional. Será apresentado também o desenvolvimento dos gradientes $\mathrm{e}$ divergentes presentes nas equações de conservação, para um sistcma cartesiano bidimensional. Considere $\mathbf{x} \in \mathbb{R}^{2}, \mathbf{x}=(x, y)^{T}, \mathbf{u} \in \mathbb{R}^{2}, \mathbf{u}=(u, v)^{T}$ e $\mathbf{g} \in \mathbb{R}^{2}, \mathbf{g}=$ $\left(g_{x}, g_{y}\right)^{T}$ os votores posição, velocidade e campo gravitacional, respectivamente. Deste modo, tem-se

$$
\begin{aligned}
& \nabla \mathbf{u}=\left(\begin{array}{ll}
\frac{\partial u}{\partial x} & \frac{\partial v}{\partial x} \\
\frac{\partial u}{\partial y} & \frac{\partial v}{\partial y}
\end{array}\right),(\nabla \mathbf{u})^{T}=\left(\begin{array}{ll}
\frac{\partial u}{\partial x} & \frac{\partial u}{\partial y} \\
\frac{\partial v}{\partial x} & \frac{\partial v}{\partial y}
\end{array}\right) \quad \mathrm{e} \\
& \nabla \cdot \mathbf{u} \quad \frac{\partial u_{i}}{\partial x_{i}} \quad \frac{\partial u}{\partial x}+\frac{\partial v}{\partial y} .
\end{aligned}
$$

Na equação (2.18), o termo do divergente pode ser escrito como

$$
\nabla \cdot\left[\mu\left(\nabla \mathbf{u}+\nabla \mathbf{u}^{T}\right)\right]=\mu \nabla \cdot\left(\nabla \mathbf{u} \mid \nabla \mathbf{u}^{T}\right)=\mu\left[\nabla \cdot(\nabla \mathbf{u})+\nabla \cdot\left(\nabla \mathbf{u}^{T}\right)\right],
$$

sabendo que

$$
\begin{aligned}
\nabla \cdot \nabla \mathbf{u} & =\nabla^{2} \mathbf{u} \\
\nabla \cdot(\nabla \mathbf{u})^{T} & =\nabla(\nabla \cdot \mathbf{u})
\end{aligned}
$$


uma. ve\% que

$$
\frac{\partial u_{i}}{\partial x_{i}}=\nabla \cdot \mathbf{u}=0
$$

e o termo Laplaciano bidimensional é definido por

$$
\nabla \cdot \nabla \mathbf{u}=\nabla^{2} \mathbf{u}=\left(\begin{array}{c}
\frac{\partial^{2} u}{\partial x^{2}}+\frac{\partial^{2} u}{\partial y^{2}} \\
\frac{\partial^{2} v}{\partial x^{2}}+\frac{\partial^{2} v}{\partial y^{2}}
\end{array}\right) .
$$

Da equação da continuidade (2.11), tem-se que

$$
\frac{\partial u}{\partial x}+\frac{\partial v}{\partial y}=0
$$

Portanto

$$
\begin{aligned}
& \frac{D u}{D t}=-\frac{\partial p}{\partial x}+\nu\left(\frac{\partial^{2} u}{\partial x^{2}}+\frac{\partial^{2} u}{\partial y^{2}}\right)+g_{x} \\
& \frac{D v}{D t}=-\frac{\partial p}{\partial y}+\nu\left(\frac{\partial^{2} v}{\partial x^{2}}+\frac{\partial^{2} v}{\partial y^{2}}\right)+g_{y}
\end{aligned}
$$

em que $\nu=\frac{\mu}{\rho_{0}}>0$ é o coeficiente de viscosidade cinemática molecular (constante) do fluido, $p$ a pressão cinemática $\left(p=\frac{p}{\rho_{0}}\right), \rho_{0}$ é a densidade e $\mu_{0}$ é o coeficiente de viscosidade. As equações (2.27) e (2.28) são as equações de quantidade de movimento nas direções $x$ e $y$ respectivamente.

\subsection{Adimensionalização}

Os problemas em mecánica dos fluidos envolvem grandezas que os caracterizam, como velocidade, pressão, massa específica, etc. Essas grandezas na forma dimensional são diferenciadas por suas magnitudes, dadas através de um sistema métrico escolhido previamente. As constantes de adimensionalização, resultantes deste processo, são importantes por caracterizarem o escoamento quanto às forças que são predominantes. As utilizadas aqui são definidas a seguir:

- Número de Reynolds (Re): Representa a razão cntre as forças inerciais (que são responsávcis pelo movimento do luido) e as forças viscosas (que são responsáveis pela dissipação devido ao coeficiente de viscosidade molecular) do escoamento, dado por

$$
R e=\frac{\rho_{0} L U}{\mu_{0}} \cdot \frac{L U}{\nu_{0}}
$$

onde, $L$ c a cscala de comprimento, $U$ é a escala de velocidade.

- Número de froude (Fr): Representa a razão entre as forças inerciais c as forģas gravitacionais, isto o

$$
F r=\frac{U}{\sqrt{g L}}
$$


Para adimensionalizar as equações de quantidade de movimento e continuidade, definem-se algumas variáveis adimensionais, como segue

$$
\rho \cdots \rho_{0} \rho^{*}, \quad \mu=\mu_{0} \mu^{*}, \quad p=\rho_{0} U^{2} p^{*}, \quad \mathbf{x}=L \mathbf{x}^{*}, \quad \mathbf{u}=U \mathbf{u}^{*}, \quad t \quad \frac{L}{U I} t^{*}, \quad \mathbf{g}=g_{0} \mathbf{g}^{*}
$$

onde $\mathbf{x}=(x, y)$.

Substituindo as variáveis adimensionais nas equações de quantidade de movimento c conservação de massa e eliminando :**" para simplificar, obtêrn-se na forma adimensional

$$
\begin{gathered}
\frac{\partial \mathbf{u}}{\partial t}+\nabla \cdot(\mathbf{u u})--\nabla p+\frac{1}{R e} \nabla^{2} \mathbf{u}+\frac{1}{F r^{2}} \mathbf{g}, \\
\nabla \cdot \mathbf{u}=0 .
\end{gathered}
$$

As equações (2.31) e (2.32) podem ser escritas na forma cartesiana cm duas dimensões como

- Conservação de massa (continuidade):

$$
\frac{\partial u}{\partial x}+\frac{\partial v}{\partial y} \cdots 0
$$

- Balanço de quantidade de movimento:

$$
\begin{aligned}
& \frac{\partial u}{\partial t}+\frac{\partial u^{2}}{\partial x}+\frac{\partial(u v)}{\partial y} \cdots-\frac{\partial p}{\partial x}+\frac{1}{R e}\left(\frac{\partial^{2} u}{\partial x^{2}}+\frac{\partial^{2} u}{\partial y^{2}}\right)+\frac{g_{x}}{F r^{2}} \\
& \frac{\partial v}{\partial t}+\frac{\partial(u v)}{\partial x}+\frac{\partial v^{2}}{\partial y}=-\frac{\partial p}{\partial y}+\frac{1}{R e}\left(\frac{\partial^{2} v}{\partial x^{2}}+\frac{\partial^{2} v}{\partial y^{2}}\right)+\frac{g_{y}}{F r^{2}}
\end{aligned}
$$

\subsection{Condições Inicial e de Contorno}

F́ fundamental para a formulação dos problemas modelados por equações diferenciais a. escolha apropriada da condição inicial e condições de contorno. A condição inicial apropriada para as equações (2.31) e (2.32) é que o campo de velocidades inicial seja especificado em todo o domínio, de modo a respeitar as condições de contorno e que seja solenoidal, isto $\grave{c}, \nabla \cdot \mathbf{u}=0$.

\subsubsection{Condições para Contornos Rígidos}

Pode-se aplicar as condições:

- Condição sem escorregamento ("no-slip"): Para escoamentos viscosos, nas paredes sólidas, define-se a componente normal $\left(u_{n}\right)$ e as componentes tangenciais $\left(u_{t}\right)$ da velocidade na parede, como sendo mulas. Esta condição reflete o fato do fluido imediatamente adjacente a parede estar cm repouso em relação a mesma. 
- Condição de simetria ou com escorregamento ("free-slip"): É usada quando há fronteiras de simetria, ou quando os efeitos da condição no-silip não são desejáveis. Neste caso define-se $u_{n} \cdots 0$ para a componente normal é fronteira rígida e $\frac{\partial u_{t}}{\partial n} \quad 0$ para as componentes tangenciais, onde $n$ é a direção normal à frontcira rígida. Essa condição permite que o fluido deslize livremente sobre a. superfície.

- Condição de entrada de fluido ("inflow"): É usada em fronteiras onde há entrada de fluido no sistema (fonte de massa). Para essa condição, define-se $u_{n}=u_{\text {infow }}$ para a componente normal da velocidade, e $u_{t}=0$ para as componentes tangenciais.

- Condição de saída de fluido ("outflow"): É usada em fronteiras onde há saída de fluido no sistema (sorvedouro de massa). Neste caso, definc-se $\frac{\partial u_{n}}{\partial n}=0$ para a componente normal da velocidade, e $\frac{\partial u_{t}}{\partial n}-0$ para as componentes tangenciais.

\subsubsection{Condições de Contorno na Superfície Livre}

Representa uma interface entre o fluido e uma atmosfera. Quando trabalha-se na superfícic livre do fluido, é necessário impor condições sobre a velocidade e a pressão |Batchelor, 1970|. 'Tais condições, considerando neste trabalho que o coeficiento de tensão superficial é nulo |Gricbel et al., 1998|, se resımem nas equações

$$
\begin{aligned}
& \mathbf{n} \cdot(\boldsymbol{\sigma} \cdot \mathbf{n})=0, \\
& \mathbf{m} \cdot(\boldsymbol{\sigma} \cdot \mathbf{n})=0,
\end{aligned}
$$

onde $\mathbf{n}$ e $\mathbf{m}$ são vetores unitários normal e tangencial à superfície livre, respectivamente e $\sigma$ é o tensor das tensões que na forma adimensional é dado por:

$$
\boldsymbol{\sigma}=-p \mathbf{I}+\frac{1}{R e}\left(\nabla \mathbf{u}+(\nabla \mathbf{u})^{T}\right) .
$$

Utilizando coordenadas bidimensionais, (2.38) pode ser escrito como:

$$
\begin{aligned}
\sigma & =\left(\begin{array}{cc}
-p & 0 \\
0 & -p
\end{array}\right)+\frac{1}{R e}\left\{\left(\begin{array}{cc}
2 \frac{\partial u}{\partial x} & \frac{\partial u}{\partial y}+\frac{\partial v}{\partial x} \\
\frac{\partial u}{\partial y}+\frac{\partial v}{\partial x} & 2 \frac{\partial v}{\partial y}
\end{array}\right)\right\} \\
& -\left(\begin{array}{cc}
-p+\frac{1}{R e}\left(2 \frac{\partial u}{\partial x}\right) & \frac{1}{R e}\left[\left(\frac{\partial u}{\partial y}+\frac{\partial v}{\partial x}\right)\right] \\
\frac{1}{R e}\left[\left(\frac{\partial u}{\partial y}+\frac{\partial v}{\partial x}\right)\right] & -p+\frac{1}{R e}\left(2 \frac{\partial v}{\partial y}\right)
\end{array}\right) .
\end{aligned}
$$

'Tomando $\mathbf{n}=\left(n_{x}, n_{y}\right)$ e $\mathbf{m}=\left(n_{y},-n_{x}\right)$ e utilizando $(2.39)$, as equaçōes (2.36) $)(2.37)$ se reduzem a.

$$
p=\frac{2}{R e}\left\{\left[\frac{\partial u}{\partial x} n_{x}^{2}+\left(\frac{\partial u}{\partial y}+\frac{\partial v}{\partial x}\right) n_{x} n_{y}+\frac{\partial v}{\partial y} n_{y}^{2}\right]\right\}
$$

e

$$
\frac{1}{R e}\left\{\left[2\left(\frac{\partial u}{\partial x}-\frac{\partial v}{\partial y}\right)\right] n_{x} n_{y}+\left(\frac{\partial u}{\partial y}+\frac{\partial v}{\partial x}\right)\left(n_{y}^{2}-n_{x}^{2}\right)\right\}=0
$$

respectivamente.

As equações (2.40) e (2.41) constituem as condições de contorno a serem satisfeitas na. superfícic livre do fluido. 


\subsubsection{Condição para a Pressão}

Fm problema do escoamento de fluidos, é importante impor condições de contorno que sejam fisicamente corretas, pois se impostas incorretamente podem gerar soluçôes fisicamente incorretas, ou fazer que o sistema de equações não tenha solução. Isto ocorre pelo forte acoplamento entre aceleração e pressão nas equações de Navior-Stokes.

Pode-se tentar deduzir condições para a pressão utilizando algumas simplificações. Considere a equação (2.18) avaliada num contorno $\Gamma$ e projetada na direção do vetor $\mathbf{n}$, normal a. I',

$$
\left.\left\{\frac{\partial(\rho \mathbf{u})}{\partial t}+\nabla \cdot(\rho \mathbf{u} \mathbf{u})\right)\right\} \cdot \mathbf{n}=\left\{-\nabla p+\nabla \cdot\left[\mu\left(\nabla \mathbf{u}+\nabla \mathbf{u}^{T}\right)\right]+\rho \mathbf{g}\right\} \cdot \mathbf{n} .
$$

Como a equação acima é avaliada no contorno l', pode-se aplicar as condições de contorno para a velocidade. Por exemplo, aplicando a condição de não escorregamento em I', ou seja, $\mathbf{u}-0$. Desconsiderando os termos mulos, tem-so:

$$
\frac{\partial(\rho \mathbf{u})}{\partial t} \cdot \mathbf{n}=\left[-\nabla p+\mu \nabla \cdot\left(\nabla \mathbf{u}+(\nabla \mathbf{u})^{T}\right) \mid \rho \mathbf{g}\right] . \mathbf{n} .
$$

Para a derivada temporal pode-se considerar que $\mathbf{u}=\mathbf{0}$ para qualquer tempo $t$ em $\Gamma$, o que resulta que o termo de derivada temporal tambóm é nulo. Assim, tom-se

$$
\nabla p \cdot \mathbf{n}=-\mu \nabla \cdot\left(\nabla \mathbf{u}+\nabla \mathbf{u}^{T}\right) \cdot \mathbf{n}+\rho \mathbf{g} \cdot \mathbf{n},
$$

o1 ainda,

$$
\frac{\partial p}{\partial n}=\mu \nabla \cdot\left(\nabla \mathbf{u}+\nabla \mathbf{u}^{T}\right) \cdot \mathbf{n}+\rho \mathbf{g} \cdot \mathbf{n} .
$$

Devido a dificuldade de se calcular exatamente o termo acima, normalmente é feita a aproximaga

$$
\mu \nabla \cdot\left(\nabla \mathbf{u}+\nabla \mathbf{u}^{r}\right) \cdot \mathbf{n}=0
$$

Assim, pode-se considerar na ausência de gravidade $\frac{\partial p}{\partial n}=0$ nos contornos rígidos. 


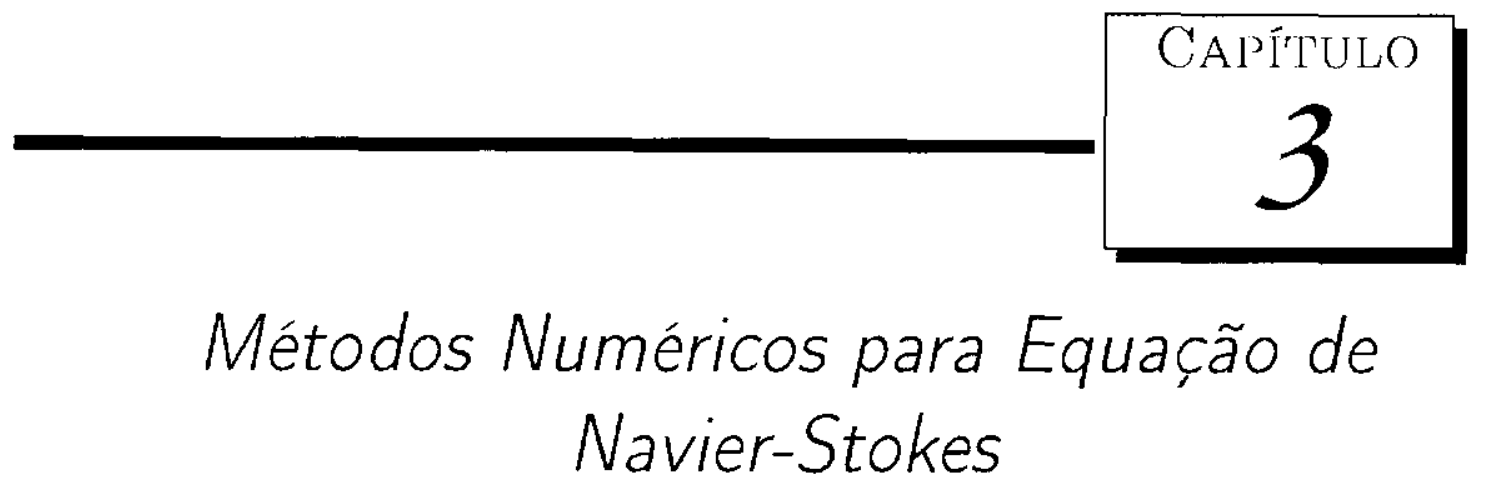

\subsection{Introdução}

Os métodos para solução das cquações de Navier-Stokes para escoamentos de fluidos podem de maneira geral, ser classificados em métodos acoplados c métodos segregados.

Métodos acoplados buscam resolver o sistema completo de equações a cada ciclo computacional, acoplando as equações de conservação de movimento e continuidade. Sendo estat a maneira mais imediata de se resolver as equaçoes de Navier-Stokes mas apresentando maiores dificuldades na sua implementação e um alto custo computacional pelas fortes influências da não-linearidade dos termos convectivos.

Neste sentido, os métodos segregados buscam o desacoplamento entre as equações, separando o sistema não-linear em problemas mais simples, que podem ser resolvidos sequencialmente.

Dentre os métodos segregados, os que mais se destacaram foram os denominados métodos da projeção. 'Tal família de métodos foi primeiramente introdızida por Chorin, seguido por muitos outros autores, como Amsdem e Ilarlow |Amsden and Harlow, 1970| com o método SMAC, Tomé e Mckee |Tomé and McKee, 1994| com o método GENSMAC e Patankar |Patankar, 1980|, |Patankar and Spalding, 1972| com o método SIMLLF. Os métodosde. projeção podem ainda ser classificados em métodos contínıos, métodos semi-discretos, o1 de passos fracionários |Gresho, 1990|, |Gresho and Chan, 1990| e métodos discretos.

Este capítulo apresenta uma brevo discussão sobre o método da projeção, a idéia do método de passo fracionário e método semi-lagrangeano o o método utilizado para discretrǐação das equaçōes de Navier-Stokes no módulo de simulação do UmFlow-2D . 


\subsection{A idéia Geral do Método da Projeção}

A teoria do método de projeção é baseada no fato de que qualquer vetor $\mathbf{v} \in \Omega$, onde $\Omega$ é um domínio com contorno $\partial \Omega$ suave, pode ser unicamente decomposto da seguinte forma

$$
\mathbf{v}=\mathbf{v}_{d}+\nabla \varphi,
$$

onde $\mathbf{v}_{d}$ é solenoidal o paralelo ao contorno $\partial \Omega$, isto é,

$$
\begin{array}{rlll}
\nabla \cdot \mathbf{v}_{d}= & 0 & \text { em } & \Omega, \\
\mathbf{v}_{d} \cdot \mathbf{n}= & 0 & \operatorname{m} & \partial \Omega,
\end{array}
$$

e $\varphi$ é um campo escalar. Da análise vetorial, tem-se $\nabla \times \nabla \varphi=0$, e portanto (3.1) é equivalente a separar o vetor $\mathrm{v}$ em componentes de divergência nula e rotacional nulo.

Para entender a teoria do método da projeção é necessário interpretar as equações de Navier-Stokes como projeções. Para simplificar este estudo, considere as equações na forma. conservativa, com $\mu$ e $\rho$ constantes em todo o domínio $\Omega$ :

$$
\begin{aligned}
& \frac{\partial \mathbf{u}}{\partial t}+\nabla \cdot(\mathbf{u u})=-\nabla p+\nu \nabla^{2} \mathbf{u} \mid \mathbf{g}, \\
& \nabla \text {. } \mathbf{u} \quad \ldots \text {. }
\end{aligned}
$$

Assim, pode-se escrever (3.4) como

$$
\frac{\partial \mathbf{u}}{\partial t}+\nabla p=S(\mathbf{u})
$$

onde,

$$
S(\mathbf{u})-\nu \nabla^{2} \mathbf{u}+\mathbf{g}-\nabla \cdot(\mathbf{u} \mathbf{u})
$$

Note que $S(\mathbf{u})$ não tem, em geral, divergência e rotacional nulos. Note ainda que

$$
\begin{aligned}
\nabla \cdot\left(\frac{\partial \mathbf{u}}{\partial t}\right)=\frac{\partial}{\partial t}(\nabla \cdot \mathbf{u}) & =0 \\
\nabla \times \nabla p & =\mathbf{0} .
\end{aligned}
$$

Segundo Chorin a equação (3.6) pode ser interpretada da seguinte forma: dado $\mathbf{u}$, o vetor $\mathrm{S}(\mathbf{u})$ e conhecido a pode ser projetado em ambos os subespaços de divergência nula $(\partial \mathbf{u} / \partial t)$ e rotacional nulo $(\nabla p)$. Matematicamente,

$$
\frac{\partial \mathbf{u}}{\partial t} \ldots \mathcal{P}[S(\mathbf{u})] ; \quad \nabla p=\mathcal{Q}[S(\mathbf{u})]
$$

ondo $\mathcal{P}$ a $\mathcal{Q}$ são operadores de projeção, que satisfazem as seguintes propriedades:

$$
\mathcal{P}^{2}=\mathcal{P} ; \quad \mathcal{Q}^{2}=\mathcal{Q} ; \quad \mathcal{P Q}-\mathcal{Q P} \ldots 0 .
$$

Note ainda que, dado $\mathbf{v}$ um vetor qualquer, $\mathcal{P}$ projeta este vetor no espaço nulo do operador divergente e $\mathcal{Q}$ o projeta no espaço nulo do operador rotacional, isto é

$$
\begin{array}{ll}
\nabla . \mathcal{P}[\mathbf{v}] \quad 0, & \forall \mathbf{v} \in \Omega \\
\nabla \cdot \mathcal{Q}[\mathbf{v}]=\mathbf{0}, & \forall \mathbf{v} \in \Omega .
\end{array}
$$


Comparando (3.8) e (3.10), obtém-se a seguinte forma para os operadores de projeção:

$$
\begin{aligned}
& \mathcal{P}=\mathbf{I}-\nabla\left(\nabla^{2}\right)^{-1}(\nabla .) \\
& \mathcal{Q}=\mathbf{I}-\mathcal{P}=\nabla\left(\nabla^{2}\right)^{-1}(\nabla .)
\end{aligned}
$$

Através destes operadores, pressão c acclcração local podem ser desacopladas das equações de Navier-Stokes. De fato, segundo Gresho |Gresho, 1990|, enquanto pressião e aceleração podem ser calculadas sequêncialmente, pressão e velocidade não, pois estão intimamente (ou fortemente) acopladas em escoamentos incompressiveis.

\subsection{Método de Projeção}

O primeiro passo do método de projeção consiste em resolver a aproximação

$$
\frac{\partial \tilde{\mathbf{u}}}{\partial t}=S(\mathbf{u})-\nabla \tilde{p}
$$

onde $\tilde{p}$ é uma aproximação para a pressão, que pode vir das condições iniciais, ou do passo anterior no algoritmo. A velocidade intermediária ũ resultante de (3.16) não é solenoidal, pois $\mathrm{cm}$ geral, $p \neq \tilde{p}$. Desta forma, $\tilde{\mathbf{u}}$ pode ser projetada no subespaço de divergência nula, utilizando-se o operador $\mathcal{P}$. Assim, uma solução solenoidal pode ser aproximada pela. projeşão

$$
\mathbf{u}_{d}=\mathcal{P}[\tilde{\mathbf{u}}]
$$

que pode ser tomada como aproximação da solução real. No entanto, o operador $\mathcal{P}$ é difícil de ser aplicado diretamente, pois $\nabla^{2}$ pode ser invertido somente através de uma função do Grecn |Jankowski, 1998|, sendo necessária a informação das condições de contorno e da geometria do problema em particular. Para evitar a inversão do operador Laplaciano, outra aproximaçăo é feita: considerando a equação (3.1) o passo de projeção pode ser feito utilizando-se a seguinte decomposição

$$
\tilde{\mathbf{u}}=\mathbf{u}_{d}+\nabla \varphi, \quad \operatorname{com} \quad \nabla \cdot \mathbf{u}_{d}=0
$$

onde $\varphi$ é o multiplicador de Lagrange associado com a projeção da solıção intermediária $\tilde{\mathbf{u}}$ no subespaço dos vetores de divergência nula $\left(\mathbf{u}_{d}\right)$ a o rotacional nulo $(\nabla \varphi)$. Como $\nabla \times \nabla \varphi=\mathbf{0}$, então $\nabla \times \tilde{\mathbf{u}}_{d} \ldots \nabla \times \mathbf{u}_{d}$, on seja, a vorticidade contida pela velocidade intermediária $\mathbf{u}$ não é alterada pela projeção. De (3.18) pode-se resolver $\mathbf{u}_{d}$ e $\varphi$ através de um procedimento de dois passos:

1. Aplicando-se o operador divergente em (3.18), pode-se encontrar $\varphi$ através da equação de Poisson

$$
\nabla^{2} \varphi=\nabla \cdot \tilde{\mathbf{u}}
$$

2. Sendo $\varphi$ conhecida, pode-se calcular $\mathbf{u}_{d}$ diretamente de (3.18) como segue

$$
\mathbf{u}_{d}=\tilde{\mathbf{u}}-\nabla \varphi .
$$

No algoritmo descrito acima, a resolução de (3.16) é chamado de passo de velocidade intermediária, a resolução de (3.18) é chamado de passo de projeção. 


\subsection{Método do Passo Fracionário}

() método de passo fracionário é um método semi-discreto, que parte das equações de Navier-Stokes já discretizadas no tempo. Por simplicidade, pode ser usado o método de Adams-Bashforth para os termos convectivos e o método trapezoidal para os termos difusivos. Assim, temos

$$
\begin{gathered}
\frac{\left(\mathbf{u}^{n+1}-\mathbf{u}^{n}\right)}{\delta t}+\left[\frac{3}{2}\left(\mathbf{u}^{n} \cdot \nabla\right) \mathbf{u}^{n}-\frac{1}{2}\left(\mathbf{u}^{n-1} \cdot \nabla\right) \mathbf{u}^{n-1}\right] \\
--\nabla p^{n \cdot 1}+\frac{1}{2 R e} \nabla^{2}\left(\mathbf{u}^{n} 1+\mathbf{u}^{n}\right), \\
\nabla \cdot \mathbf{u}^{n+1}-0 .
\end{gathered}
$$

A idéia é aproximar (3.21) por uma velocidade tentativa $u^{*}$, usando a equação da quantidade de movimento sem o termo da pressão, e utilizar a pressão para projetar a velocidade tentativa no espaço de funçoes incompressiveis discretas encontrando a velocidade final. Matomaticamente, este processo consiste em fazer a scguinte separação

$$
\begin{gathered}
\frac{\mathbf{u}^{*}-\mathbf{u}^{n}}{\delta t}+\cdot\left[\frac{3}{2}\left(\mathbf{u}^{n} \cdot \nabla\right) \mathbf{u}^{n}-\frac{1}{2}\left(\mathbf{u}^{n \cdots 1} \cdot \nabla\right) \mathbf{u}^{n-1}\right]=\frac{1}{2 R e} \nabla^{2}\left(\mathbf{u}^{*}+\mathbf{u}^{n}\right), \\
\frac{\mathbf{u}^{n+1}-\mathbf{u}^{*}}{\delta t} \cdots-\nabla p^{n+1} .
\end{gathered}
$$

$\Lambda$ pressão em (3.24) é encontrada aplicando-se o operador divergente e pela condição de incompressibidade (3.22). Resultando na equação de Poisson para a pressão dada por

$$
(\nabla \cdot \nabla) p^{n+1}-\cdots \cdot \frac{1}{\delta t} \nabla \cdot \mathbf{u}^{*}
$$

Assim essas equações são resolvidas na sequência (3.23), (3.25) e (3.24) em cada passo de tempo. Mais detalhes podem ser obtidos em |Perot, 1993| e |Armfield and Street, 2002|.

\subsection{Método Semi-Lagrangeano}

A idéia básica do método semi-lagrangeano é acompanhar uma partícula de fluido durante sua trajetória ao longo da malha sobre o escoamento. Seja $\varphi$ uma variável

$$
\frac{D \varphi}{D t}=\mathbf{F}
$$

Utilizando, o referencial Lagrangeano, a derivada material $\frac{D \varphi}{D t}$ pode ser aproximada por

$$
\left.\frac{\varphi(\mathbf{x}, t \cdot \delta t)-\varphi(\mathbf{x}}{-} \delta \mathbf{x}, t\right) \delta t=\mathbf{F}
$$

onde $\mathrm{x}$ é a posição de uma partícula arbitrária $p_{a}$ no tempo $t+\delta t$, e

$$
\delta \mathbf{x}=\mathbf{u} \delta t
$$


Assim,

$$
\varphi(\mathbf{x}, t+\delta t)=\varphi(\mathbf{x}-\mathbf{u} \delta t, t)=\delta t \mathbf{F} .
$$

Note que $\mathbf{x}$ é a posição ocupada no tempo $t+1 \delta t$ por uma partícula de fluido $p_{a}$ que ocupava a posição $\mathbf{x}-\delta \mathbf{x}$ no tempo $t$.

O termo $\varphi(\mathbf{x}-\delta \mathbf{x})$ ć calculado usando uma interpolação linear para $\varphi$ na posição $(\mathbf{x}-\delta \mathbf{x})$. Se $(\mathbf{x}-\delta \mathbf{x})$ estiver sobre uma aresta a interpolação é efetuada utilizando os vértices desta aresta, caso $(\mathbf{x}-\delta \mathbf{x})$ cstiver no interior de um triângulo, a interpolação é efetıada utilizando os vértices do triângulo.

Para o caso da posição $(\mathbf{x}-\delta \mathbf{x})$ estar dentro do elemento triangular (4.2), tal função de interpolação deve, a partir do conhecimento de u nos vértices do triângulo, permitir o cálculo de u e suas derivadas em qualquer posição dentro do elemento triangular.

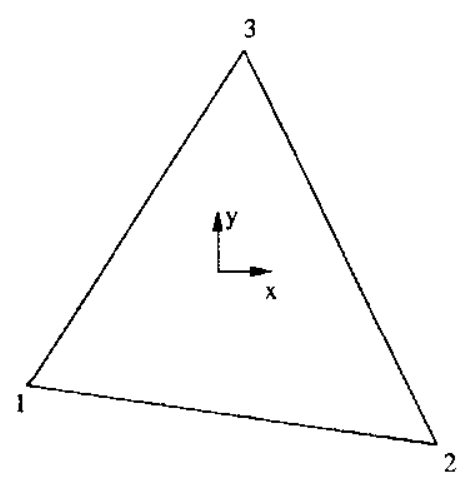

Figura 3.1: Elemento triangular.

A função de interpolação escolhida para u é dada por

$$
\mathbf{u}=\lambda_{1} \mathbf{u}_{1}+\lambda_{2} \mathbf{u}_{2}+\lambda_{3} \mathbf{u}_{3} .
$$

Com os valores de $\mathbf{u}_{1}, \mathbf{u}_{2}$ e $\mathbf{u}_{3}$ e os valores das coordenadas $(x, y)$ nos pontos $1,2,3 \mathrm{e}$ a posição $\left(x^{\star}, y^{\star}\right)=(x-\delta x, y-\delta y)$, é possível encontrar os valores das constantes $\lambda_{1}, \lambda_{2} \mathrm{e}$ $\lambda_{3}$, da seguinte maneira

$$
\begin{gathered}
1=\lambda_{1}+\lambda_{2}+\lambda_{3}, \\
x^{\star}=\lambda_{1} x_{1}+\lambda_{2} x_{2}+\lambda_{3} x_{3}, \\
y^{\star}=\lambda_{1} y_{1}+\lambda_{2} y_{2}+\lambda_{3} y_{3} .
\end{gathered}
$$

Essas expressões podem ser escritas como o seguinte sistema linear

$$
\left(\begin{array}{ccc}
1 & 1 & 1 \\
x_{1} & x_{2} & x_{3} \\
y_{1} & y_{2} & y_{3}
\end{array}\right)\left(\begin{array}{c}
\lambda_{1} \\
\lambda_{2} \\
\lambda_{3}
\end{array}\right)=\left(\begin{array}{c}
1 \\
x^{\star} \\
y^{\star}
\end{array}\right)
$$

cuja solução resulta nos valores para as constantes $\lambda_{1}, \lambda_{2}$ e $\lambda_{3}$.

Para o (aso da nova posiçāo estar sobre uma aresta a função de interpolação deve, a partir do conhecimento de u nos vértices da aresta, permitir o cálculo de u e suas derivadas em qualquer posição da aresta. A função de interpolação escolhida para u é

$$
\mathbf{u}-\lambda_{1} \mathbf{u}_{\mathbf{1}}+\lambda_{2} \mathbf{u}_{\mathbf{2}}
$$


Como no caso anterior, com os valores de $\mathbf{u}_{1}$ e $\mathbf{u}_{2}$ e os valores das coordenadas $(x, y)$ nos pontos 1,2 c na posição $(\mathbf{x}-\mathbf{u} \delta t)=\left(x^{\star}, y^{\star}\right)$ é possível encontrar os valores das constantes $\lambda_{1}$ e $\lambda_{2}$.

\subsection{Método Utilizado no UmFlow-2D}

O método numérico utilizado para simular escoamentos Newtonianos em malhas não estruturas estão baseados nos métodos da projeção, na formulação semi-lagrangeana para os termos convectivos e no método de diferenças finitas generalizadas. Fstas três técnicas reunidas dão um aspecto inovador para o trabalho aqui desenvolvido.

Assim, dadas as equações de conservação

$$
\begin{gathered}
\frac{D \mathbf{u}}{D t}=-\nabla p+\frac{1}{R e} \nabla^{2} \mathbf{u}+\frac{1}{F r^{2}} \mathbf{g}, \\
\nabla \cdot \mathbf{u}=0 .
\end{gathered}
$$

Para a aproximação da derivada material em (3.35), é utilizado o método semi-lagrangeano, visto na seção (3.5). Assim

$$
\frac{D \mathbf{u}}{D t}=\frac{\mathbf{u}(\mathbf{x}, t \mid \delta t)-\mathbf{u}(\mathbf{x}-\delta \mathbf{x}, t)}{\delta t} .
$$

Substituindo (3.37) em (3.35), têm-se

$$
\frac{\mathbf{u}(\mathbf{x}, t \mid \delta t)-\mathbf{u}(\mathbf{x}-\delta \mathbf{x}, t)}{\delta t}-\nabla p+\frac{1}{R e} \nabla^{2} \mathbf{u}+\frac{\mathbf{g}}{F r^{2}} .
$$

Litilizando o método dos passos fracionários, descrito na seção (3.4), na equação (3.38), obtém-se

$$
\begin{gathered}
\frac{\tilde{\mathbf{u}}(\mathbf{x}, t+\delta t)-\mathbf{u}(\mathbf{x}-\delta \mathbf{x}, t)}{\delta t}=\frac{1}{R e} \nabla^{2} \mathbf{u}+\frac{1}{F r^{2}} \mathbf{g}, \\
\frac{\mathbf{u}(\mathbf{x}, t+\delta t)-\tilde{\mathbf{u}}(\mathbf{x}, t+\delta t)}{\delta t}=-\nabla p, \\
\nabla^{2} p \cdot \frac{1}{\delta t} \nabla \cdot \tilde{\mathbf{u}}(\mathbf{x}, t+\delta t) .
\end{gathered}
$$

Essa estratégia de resolução pode ser descrita nos seguintes passos:

- Passo 1: Cálculo das velocidades intermediárias

$$
\tilde{\mathbf{u}}=-\mathbf{u}^{*}+\delta t\left(\frac{1}{R e} \nabla^{2} \mathbf{u}+\frac{1}{F r^{2}} \mathbf{g}\right)
$$

onde $\mathbf{u}^{*}=\mathbf{u}(\mathbf{x}-\delta \mathbf{x}, l)$

- Passo 2: Cálculo da pressão

$$
\nabla^{2} p^{n+1}=\frac{1}{\delta t} \nabla \cdot \tilde{\mathbf{u}}
$$

utilizando as seguintes condiçōes de contorno: 
- Condiçoes homogêneas do tipo Neumann no contorno rígido, ou seja,

$$
\frac{\partial p^{n+1}}{\partial n}-0
$$

Fsta condição é utilizada também, nas regiôes de entrada de fluido, caso existam.

- Condições homogêneas do tipo Dirichlet na superfície livre, ou seja,

$$
p^{n+1}=0 .
$$

Esta condição é também utilizada nas regiões de saída de fluido, caso existam.

- Passo 3: Atualizar o campo de velocidade final

$$
\mathbf{u}^{n+1}=\tilde{\mathbf{u}}-\delta t \nabla p^{n\urcorner 1}
$$

Desta forma, esses passos formam um ciclo computacional, a partir de um tempo inicial $t_{n}$ para o cálculo das variáveis primitivas num tempo posterior $t_{n+1}=t_{n}+\delta t$.

\subsubsection{Classificação das Células}

O módulo de simulação do ambiente UmFlow-2D foi originalmente criado para resolver problemas com superfícies livres, deste modo, e necessário classificar as células da malha, pois o fluido está continuamente em movimento. Ou seja, a cada passo no tempo o fluido se movimenta. Essa classificação, está baseada na classilicação do método GENSMAC, e identifica se a célula fa\% parte da entrada ou saída do domínio ou está no contorno rígido, se contém ou não fluido, se pertence à superfície livre. Aqui é considerado como domínio apenas os elementos triangulares interno ao domínio rígido, os elementos triangulares pertencentes ao domínio rígido, à fronteira de entrada e saída do fluido são fictícios considerados apenas para aplicações de condições de contorno. Para tanto se adota a seguinte classificação:

- Células vazias ("empty") (E): São elementos triangulares que não contém fluido;

- Células cheias ("full") (F): São elementos triangulares cheios de fluido e não possuem nenhuma face(aresta) em contato com as células vazias;

- Células de superfície ("surface") (S): São elementos triangulares que contém fluido mas possuem uma ou mais faces (arestas) em contato com células vazias;

- Células do contorno ("boundary") (B): São elementos triangulares que possucm uma aresta pertencente ao domínio rígido e não pertencem ao domínio;

- Células do injetor ("inflow") (I): São elementos triangulares que possuem uma aresta pertencente à fronteira de entrada do fluido no domínio e não pertencem ao domínio;

- Células do ejetor ("outflow") (O): São elementos triangulares que possuem uma aresta pertencente à fronteira de saída do fluido no domínio e não pertencem ao domínio.

Atć o momento apenas simulações de fluido com escoamento confinado está implernentado, assim não é encontrado células vazias o de superfícies livres.

A figura (3.2) ilıstra essa classificação das células na malha em um instante dado para um escoamento bidimensional. 


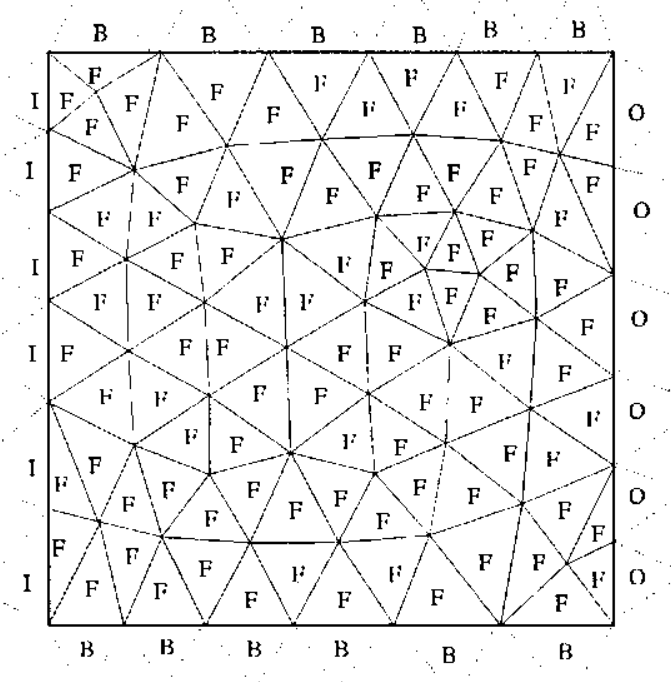

Figura 3.2: 'lipos de células.

\subsubsection{Condições Inicial e de Contorno}

As condições de contorno utilizadas são as mesmas apresentadas na seção 2.6 .

\subsubsection{Condição de Estabilidade do Método}

Assim como no método GENSMAC, o método 11tilizado no módulo de simulação do UmFlow-2D, lida com equaçōes não-lineares, dificultando a análise analítica da estabilidade. Desta forma, pode-se argumentar pelo estudo das equações linearizadas utilizando um método de análise de estabilidade. Portanto o critério de estabilidade empregado é uma condição necessária mas não suficiente.

O uso de integração temporal explícita impõe severas restrições aos valores permitidos de $\delta t$ em problemas onde os termos viscosos da equação (2.12) são predominantes. Em particular, esse tipo de problema ocorre quando o escoamento apresenta nímero de Reynolds baixo, os chamados creep flow.

A restrição imposta pelo tratamento explícito dos termos viscosos é

$$
\delta t_{v i s c} \leq \frac{R e}{2}\left(\frac{1}{(h)^{2}} \mid \frac{1}{(h)^{2}}\right)^{-1}
$$

onde hé igual ao comprimento da menor aresta da malha.

Outra restrição de estabilidade do método relaciona o valor do passo temporal com o espaçamento da malha e com uma velocidade de referencia. Ou seja, o fluido ao longo do escoamento, não pode percorrer uma distància maior que o comprimento de um elemento triang11lar a cada passo no tempo, pois caso isso ocorra, pode-se perder informaçòes sobre as propriedades que estão sendo transportadas. Fssa restrição, exige que

$$
\delta t_{C F L_{x}} \leq \frac{1}{2}\left(\frac{h}{|u|_{\max }}\right)
$$


e

$$
\delta t_{C F L_{y}} \leq \frac{1}{2}\left(\frac{h}{|v|_{\max }}\right)
$$

onde $|u|_{\max }$ e $|v|_{\max }$ são os módulos máximos das velocidades nas direções $x$ e $y$, respectivamente.

Essa restrição é derivada da condição Courant-H'riedrichs-Lewy (CFL) |Courant et al., 1967|, que exige que

$$
\eta_{x} \leq 1 \text { e } \eta_{y} \leq 1
$$

onde $\eta_{x}$ e $\eta_{y}$, são os números de Courant. Para $a=2|u|_{\max }$ e $b=2|v|_{\max }$ determina-se: a condiçăo $(3.46)$ e (3.47).

De acordo com |Tomé and McKee, 1994|, |Griebel et al., 1998| o|Fortuma, 2000|, um fator de segurança Fact, tal que Fact $\in(0,1]$, deve ser aplicado nas restrições (3.18), (3.46) e (3.47).

Finalmente, o valor de $\delta t$ utilizado na simulação, deve satisfazer simultaneamente as restriçōes (3.18), (3.46) e (3.47), e multiplicado pelo fator de segurança, ou seja,

$$
\delta t=F a c t \cdot \min \left(\delta t_{v i s c}, \delta t_{C H^{\prime} L_{x}}, \delta t_{C F L_{y}}\right) .
$$

Outras expressões para o critério de estabilidade podem ser adotadas em metodologias com natureza explícita da integração temporal. 


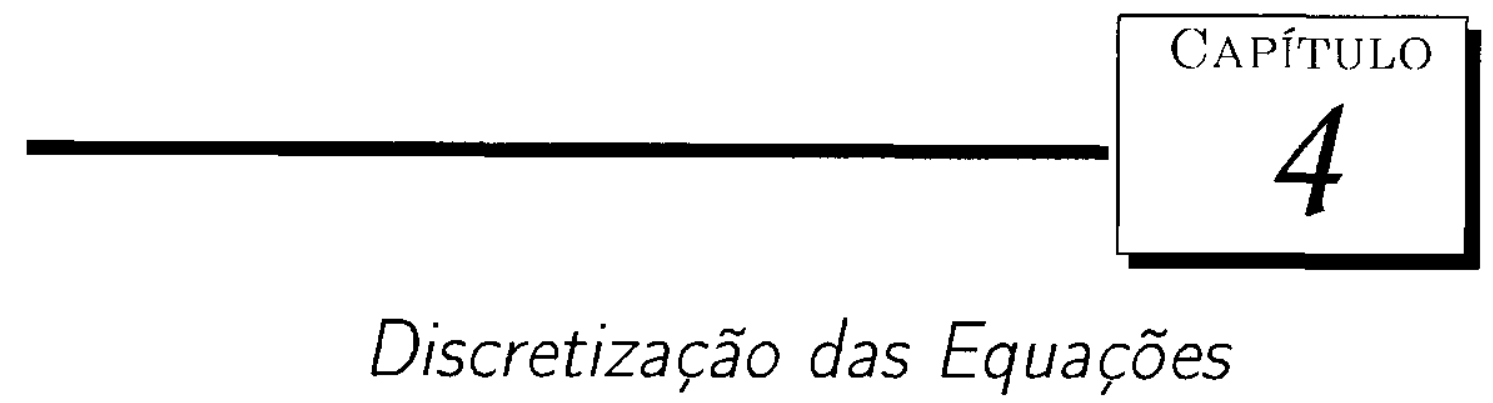

\subsection{Introdução}

A solução numérica de $11 \mathrm{~m}$ problema de escoamento de fluidos pode ser obtida seguindo as seguintes etapas: dado um problema físico, utiliza-se modelagem matemática através de equaçôs governantes e em scguida obtém-se uma solıção aproximada aplicando um processo de discretização. Após obter essa solıção são feitas análises e interpretações dos resultados numóricos, muitas vezes usando técnicas de visualização que em diversos casos, permite uma análise qualitativa rápida e direta. Portanto, $1 \mathrm{~m}$ processo muito importante e fundamental na $\mathrm{DFC}$, é escolher de forma apropriada a técnica de resolıção das equações governantes e um ambiente de simulação. Esse ambiente, deve unir técnicas de modelagem do problema, resolução das equaçóes e visualização dos resultados.

No presente: trabalho, utiliza-se os métodos passo fracionário e semi-lagrangano para o método de resolução das equações de Navier-Stokes, e utiliza-se o soltware UmFlow-2D, desenvolvido neste trabalho, para o ambiente de simulação.

A malha utilizada é triangularizada, como mostra a figura 4.1 e a estruturada de dados SHE, para a representação de malhas não estruturadas em duas dimensões. Nesse tipo de malha, a numeração das incógnitas na cólula, como a pressão (ou outra componente $\phi$ do fluido), seguc a mesma numeração da célula, e as velocidades, a numeração dos vértices.

A scguir, as cquações do método na forma discreta e a estruturas de dados SHE, serão apresentadas.

\subsection{Discretização das Equações}

Para expressar de forma adequada o tratamento computacional de um modelo utiliza-se o processo do discretização, que ć dividido em duas fases:

1. Discretização de um domínio: A solução numérica das EDPs não pode ser obtida sobre $11 \mathrm{~m}$ domínio contínuo devido aos infinitos pontos que o constituem. Assim, faz-se uma representação mumérica dessa regiào, incluindo as posições dos pontos onde 


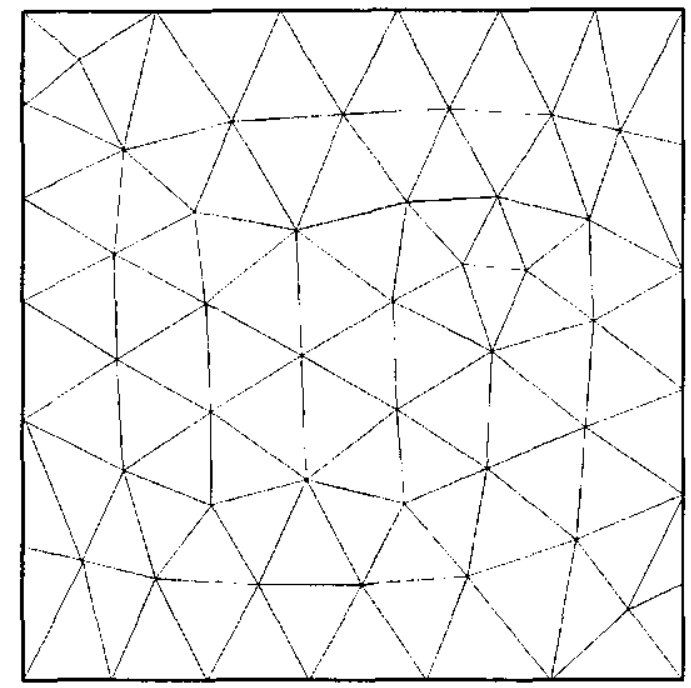

Figura 4.1: Malha triangularizada.

deseja-se aproximar a solıção. Assim, o domínio é dividido em um número finito de regiões discretas, que sũo chamadas de células computacionais, ou seja o domínio é discretizado. Quando o problema é transiente, o intervalo de tempo é dividido em passos temporais.

2. Discretização das equações: Na segunda fase, os termos que compõem as equações são escritos cm função dos valoress das incógnitas em pontos discretos adjacentes. A discretização das equações, fornece equaçóes de diferenças relacionadas as variáveis dependentes nos pontos do domínio discretizado. Nessa fase, introduzcm-so as condiçóes de contorno do problema, normalmente modificando-se as equacóes em pontos proximos da fronteira.

A discretização das equaçoes de conservagão será feita sobre uma malha triangular, as velocidades na direção $x$ e na direção $y$ são discretizadas sobre os vértices de cada triângulo, a pressão é discretizada no centro dos triângulos (4.2).

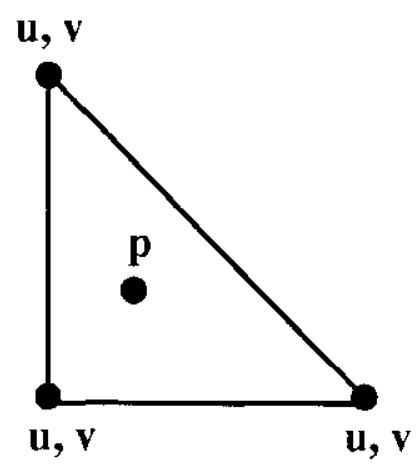

Figura 4.2: Célula Comprutacional. 


\subsubsection{Aproximação das Velocidades}

Para o cálculo das componentes da velocidade $\tilde{u}, \tilde{v}$

$$
\tilde{\mathbf{u}}=\mathbf{u}^{*}+\frac{\delta t}{R e}\left\{\frac{\partial^{2} \mathbf{u}}{\partial \mathbf{x}^{2}}+\frac{\partial^{2} \mathbf{u}}{\partial y^{2}}\right\}+\delta t\left\{\frac{1}{F r^{2}} \mathbf{g}\right\}
$$

onde $\mathbf{u}^{*}=\mathbf{u}(\mathbf{x}-\delta \mathbf{x}, t)$.

As derivadas em (4.1) é aproximado pelo método DFG utilizando aproximação por mínimos quadrados, em células do tipo $\mathrm{C}(\mathrm{I}), \mathrm{C}(\mathrm{II})$ on $\mathrm{C}(\mathrm{HI})$ dependendo do número de triângulos vizinhos utilizando uma função polinomial de grau 2. Seja o polinômio de aproximação dado por:

$$
\begin{aligned}
W_{0}= & c_{1} P^{(1)}+c_{2} P^{(2)}+c_{3} P^{(3)}+c_{4} P^{(4)}-\nmid c_{5} P^{(5)} \\
& c_{1_{\mathbf{u}}} x+c_{\mathbf{2}_{\mathbf{u}}} \bar{y}+c_{3_{\mathrm{u}}} \bar{x}^{2}+c_{4_{\mathbf{u}}} \bar{x} \bar{y}+c_{5_{\mathbf{u}}} \bar{y}^{2}
\end{aligned}
$$

Resolvendo o sistema linear em (1.6) de ordem 5, tem-se

$$
\frac{\partial^{2} \tilde{u}}{\partial x^{2}}+\frac{\partial^{2} \tilde{u}}{\partial y^{2}}=2 c_{3 \tilde{u}}+2 c_{5 \tilde{u}}
$$

Desta maneira, para o cálculo das componentes da velocidade $\tilde{u}, \tilde{v}$ tem-se:

$$
\begin{aligned}
& \tilde{u}_{v_{i}}^{n+1}=u^{*}+\delta t\left(\frac{1}{R e}\left(2 c_{3 \tilde{u}}+2 c_{5 \tilde{u}}\right)+\frac{g_{x}}{F r^{2}}\right), \\
& \tilde{v}_{v_{i}}^{n+1}=v^{*}+\delta t\left(\frac{1}{R e}\left(2 c_{3 \tilde{v}}+2 c_{5 \tilde{v}}\right)+\frac{g_{y}}{F r^{2}}\right),
\end{aligned}
$$

em que $u^{*}, v^{*}$ são as componentes de velocidades, obtida pela aproximação da derivada material pelo método semi-lagrangeano, calculadas por interpolação no tempo $t$.

\subsubsection{Aproximação da Equação de Poisson para a Pressão}

Para a equação de Poisson

$$
\frac{\partial^{2} p}{\partial x^{2}}+\frac{\partial^{2} p}{\partial y^{2}}=\tilde{D}
$$

da mesma maneira, que no cálculo das derivadas da velocidade, mas agora utilizando células do do tipo C(IV), aproxima-se as derivadas de $p$ pela função polinomial de graı 2 , resultando num sistema linear (1.6), de ordem 5. Assim, para cada célıla $C_{i}$ da malha temos o sistema, $\mathbf{A c}=\mathbf{b}$, onde:

$$
A-\left(\begin{array}{ccccc}
\sum_{v_{k} \in S_{i}}\left(x_{k} x_{k}\right) \omega_{k} & \sum_{v_{k} \in C_{i}}\left(x_{k} y_{k}\right) \omega_{k} & \sum_{v_{k} \in C_{i}}\left(x_{k} x_{k}^{2}\right) \omega_{k} & \sum_{v_{k} \in C_{i}}\left(x_{k} x_{k} y_{k}\right) \omega_{k} & \sum_{v_{k} \in C_{i}}\left(x_{k} y_{k}^{2}\right) \omega_{k} \\
\sum_{v_{k} \in C_{i}}\left(y_{k} x_{k}\right) \omega_{k} & \sum_{v_{k} \in C_{i}}\left(y_{k} y_{k}\right) \omega_{k} & \sum_{v_{k} \in C_{i}}\left(y_{k} x_{k}^{2}\right) \omega_{k} & \sum_{v_{k} \in C_{k}}\left(y_{k} x_{k} y_{k}\right) \omega_{k} & \sum_{v_{k} \in C_{i}}\left(y_{k} y_{k}^{2}\right) \omega_{k} \\
\sum_{v_{k} \in C_{i}}\left(x_{k}^{2} x_{k}\right) \omega_{k} & \sum_{v_{k} \in C_{i}}\left(x_{k}^{2} y_{k}\right) \omega_{k} & \sum_{v_{k} \in C_{i}}\left(x_{k}^{2} x_{k}^{2}\right) \omega_{k} & \sum_{v_{k} \in C_{i}}\left(x_{k}^{2} x_{k} y_{k}\right) \omega_{k} & \sum_{v_{k} \in C_{i}}\left(x_{k}^{2} y_{k}^{2}\right) \omega_{k} \\
\sum_{v_{k} \in C_{i}}\left(x_{k} y_{k} x_{k}\right) \omega_{k} & \sum_{v_{k} \in C_{i}}\left(x_{k} y y_{k}\right) \omega_{k} & \sum_{v_{k} \in C_{i}}\left(x_{k} y_{k} x_{k}^{2}\right) \omega_{k} & \sum_{v_{k} \in C_{i}}\left(x_{k} y_{k} x_{k} y_{k}\right) \omega_{k} & \sum_{v_{k} \in C_{i}}\left(x_{k} y_{k} y_{k}^{2}\right) \omega_{k} \\
\sum_{v_{k} \in C_{i}}\left(y_{k}^{2} x_{k}\right) \omega_{k} & \sum_{v_{k} \in C_{i}}\left(y_{k}^{2} y_{k}\right) \omega_{k} & \sum_{v_{k} \in C_{i}}\left(y_{k}^{2} x_{k}^{2}\right) \omega_{k} & \sum_{v_{k} \in C_{i}}\left(y_{k}^{2} x_{k} y y_{k}\right) \omega_{k} & \sum_{v_{k} \in C_{i}}\left(y_{k}^{2} y_{k}^{2}\right) \omega_{k}
\end{array}\right)
$$


c

$$
b=\left(\begin{array}{c}
\sum_{v_{k} \in C_{i}}\left(p_{k} \cdot p_{i}\right) x_{k} \omega_{k} \\
\sum_{v_{k} \in C_{i}}\left(p_{k} \cdot p_{i}\right) y_{k} \omega_{k} \\
\sum_{v_{k} \in C_{i}}\left(p_{k}-p_{i}\right) x_{k}^{2} \omega_{k} \\
\sum_{v_{k} \in C_{i}}\left(p_{k}-p_{i}\right) x_{k} y_{k} \omega_{k} \\
\sum_{v_{k} \in C_{i}}\left(p_{k}-p_{i}\right) y_{k}^{2} \omega_{k}
\end{array}\right)
$$

em que o número do nós da célula $\left(v_{k} \in C_{i}\right)$ deve ser maior que o número de monômios do polinômio evitando que o sistema seja indeterminado. E nesse caso ve é o vértice central do elemento triangular.

Lombrando que

$$
\frac{\partial^{2} p}{\partial x^{2}}=2 c_{3 p} \quad \text { e } \quad \frac{\partial^{2} p}{\partial y^{2}}=2 c_{5 p}
$$

Para a equação (4.5), temos

$$
2 c_{3 p}+2 c_{5 p}=\tilde{D}
$$

onde o termo do lado direto na equação (4.8) é calculado no centro dos elementos triangulares e

$$
\tilde{D}=\tilde{D}_{C_{i}}=2 c_{1 \tilde{u}}+2 c_{2 \tilde{v}}
$$

ó calculado por uma interpolação linear dos vértice do elemento triangular central da cólula $C_{i}$.

() sistema $\mathbf{A c}=\mathbf{b}$, pode ser transformado no seguinte sistema

$$
\left(\begin{array}{ccccc}
a_{1,1} & a_{1,2} & a_{1,3} & a_{1,4} & a_{1,5} \\
& a_{2,2} & a_{2,3} & a_{2,4} & a_{2,5} \\
& & a_{3,3} & a_{3,4}^{\prime} & a_{3,5}^{\prime} \\
& & & a_{4,4} & a_{4,5}^{\prime} \\
& & & & a_{5,5}^{\prime}
\end{array}\right)\left(\begin{array}{c}
c_{1 p} \\
c_{2 p} \\
c_{3 p} \\
c_{4 p} \\
c_{5 p}
\end{array}\right)=\left(\begin{array}{c}
b_{1} \\
b_{2} \\
b_{3} \\
b_{4} \\
b_{5}
\end{array}\right) .
$$

Obtendo os passos inversos da triangularização, manipulando o termo independente de modo que os termos $p_{i}$ e $p_{k}$ estejam em evidência, podemos escrever cada um dos termos $c_{3 p}$ e $c_{5 p}$ como uma relação lincar dos valores de $p$ no nó $v_{i}$ do triângulo central e nos nós $v_{k}$ pertencentes a célula $\left(C_{i}\right)$,

$$
\begin{aligned}
& c_{3 p}=\sum_{v_{k} \subset C_{i}}{\overline{\alpha_{i, i}}}^{3} p_{i}+\sum_{v_{k} \in C_{i}} \alpha_{i, k}^{\overline{3}} p_{k}, \\
& c_{5_{p}}=\sum_{v_{k} \in C_{i}}{\overline{i_{i, i}}}^{5} p_{i}+\sum_{v_{k} \subset C_{i}} \alpha_{i, k}^{-5} p_{k} .
\end{aligned}
$$

Então, o operador $\nabla^{2} p$, aplicado na célula $C_{i}$, pode ser escrito por:

$$
\begin{aligned}
\nabla^{2} p_{C_{i}} & -\sum_{v_{k} \in C_{i}} 2\left(\bar{\alpha}_{i, i}^{3}+\bar{\alpha}_{i, i}^{5}\right) p_{i}+\sum_{v_{k} \in C_{i}} 2\left(\alpha_{i, k}^{3}+\bar{\alpha}_{i, k}^{\bar{s}}\right) p_{k} \\
& \alpha_{i, i} p_{i}
\end{aligned}
$$


onde

$$
\alpha_{i, j}=2\left(\alpha_{i, j}^{3}+\alpha_{i, j}^{5}\right) \quad \text { para } \quad j=i, k \text {. }
$$

Assim

$$
\alpha_{i, i} p_{i}+\sum_{v_{k} \in C_{i}} \alpha_{i, k} p_{k} 2\left(\alpha_{i, j}^{3}+\alpha_{i, j}^{5}\right) \quad \text { para } j \cdots i, k .
$$

Proposição: Os termos $\alpha_{i, i}$ e $\alpha_{i, k}$ associados a $v_{i}$ e $v_{k} \in C_{i}$, cumprem a seguinte desigualdade:

$$
\left|\alpha_{i, i}\right| \leq \sum_{v_{k} \in C_{i}}\left|\alpha_{i, k}\right|
$$

A equação (4.12) quando aplicada em todos os elementos triangulares do domínio gera. um sistema linear para $p$, cuja matriz é esparsa e não simétrica.

$$
\begin{aligned}
m_{1,1} p_{1}+m_{1,2} p_{2}+\cdots+m_{1, n_{v}} p_{n_{v}} & =\tilde{D}_{C_{1}} \\
m_{2,1} p_{1}+m_{2,2} p_{2}+\cdots+m_{2, n_{v}} p_{n_{v}} & =\tilde{D}_{C_{2}} \\
& \vdots \\
m_{n_{C}, 1} p_{1}+m_{n_{C}, 2} p_{2}+\cdots+m_{n_{C}, n_{v}} p_{n_{C}} & =\tilde{D}_{C_{n^{2} C}},
\end{aligned}
$$

onde $m_{i, i}=-\alpha_{i, i}, m_{i, k}=-\alpha_{i, k}$ para $v_{k} \in C_{i}$, e os demais elementos matriciais são nulos.

Para a discretização de, $p$ na região de contorno é introduzido, para cada elemento triangllar pertencente ao contorno, um elemento triangular "fantasma", aumentando uma vizinhança na célıla de aproximação das derivadas.

Na região de entrada de fluido e no contorno rígido utiliza-se condições homogêneas do tipo Neumann, o11 seja,

$$
\frac{\partial p}{\partial n}=0
$$

$o$ valor de $p$ no elemento fantasma é igual ao valor de $p$ no elemento triangular de contorno. O termo independente $b$ em (1.6) não é alterado.

Para região de saída de fluido são utilizadas condições homogêneas do tipo Dirichlet, olı seja,

$$
p=0 .
$$

Neste caso o valor de $p$ no elemento fantasma é igual ao valor de $-p$ no elemento triangular de contorno. Para o termo independente $b$ em (1.6) é somado $2 p_{0} P_{f}^{i}$ onde $P_{f}^{i}$ é o monomio $i$ do clemento triangular fantasma. Seja a nımeração das células como na figıra (4.3), a linha da matriz para essa célula deve ser

$$
\left[\begin{array}{llllllllllllllll}
\alpha_{1,1} & \alpha_{1,2} & \alpha_{1,3} & \alpha_{1,4} & \alpha_{1,5} & \alpha_{1,6} & \alpha_{1,7} & \alpha_{1,8} & 0 & \alpha_{1,10} & 0 & \alpha_{1,12} & 0 & \alpha_{1,14} & \alpha_{1,15} & \alpha_{1,16}
\end{array}\right]
$$

Concluimos que, para a matriz, não possuir elementos negativos na diagonal principal, o domínio deve ser triangularizado de modo que um elemento triangular tenha apenas uma aresta pertence ao seu contorno. 


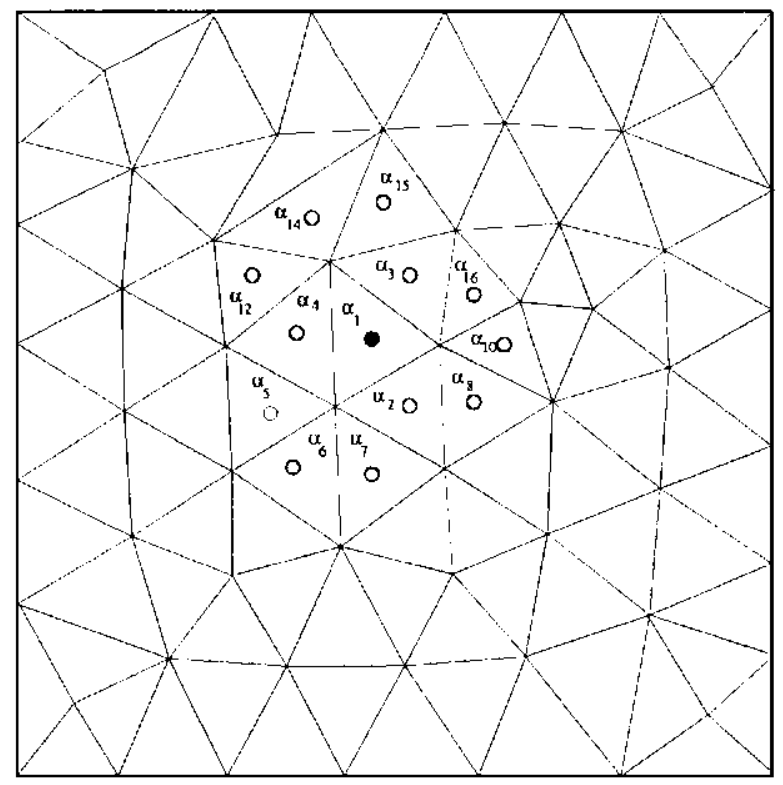

Figura 4.3: Célula com triângulos numerados.

\subsubsection{Técnicas de Armazenagem}

Como discutido na seção 4.2.2, a discretização da equação (4.5) resulta uma matriz $\mathbf{A}$ esparsa e não simétrica para as equações no cálculo de $p$. Desta forma, foi necessário o uso de técnicas de armazenagem compacta para essa matriz. Existem várias técnicas de armazenamento, como as descritas em |Knuth, 1968| e |Bentley, 1986|. No presente trabalho, ć utilizada a técnica de indexaçào por linhas descrita por Bentley |Bentley, 1986|.

Para representar a matriz $\mathrm{A}$ de dimensão $n \times n$, em que $n$ é o número de células do domínio, a técnica de indexação por linhas necessitá de dois vetores unidimensionais, sa e ija. A seguir, um resumo do esquema para aplicação desse técnica é apresentado.

1. As primeiras $n$ posições do vetor sa armazenam os elementos da diagonal principal da. matriz, $\mathbf{A}$;

2. $A$ posição $n+1$ do vetor sa é um valor qualquer, aqui denotado $x$;

3. As posições $>n+1$ do vetor sa armazena os demais elementos da matriz, percorrendo-a por linha;

4. As primeiras $n$ posições do vetor ija armazenam os índices do vetor sa nos quais estão armazenados o primeiro elemento fora da diagonal principal de cada linha;

5. A primeira posição do vetor ija é sempre igual a $n+2$;

6. As posições $>n+1$ do vetor i ja armazenam o número da coluna em que os elementos fora da diagonal pertencem, percorrendo a matriz por linha. 
Como exemplo da aplicação dessa técnica, considere a matriz.

$$
\mathbf{A}=\left(\begin{array}{lllll}
2 & 0 & 2 & 0 & 0 \\
0 & 3 & 4 & 0 & 0 \\
0 & 5 & 6 & 7 & 0 \\
0 & 0 & 0 & 8 & 9 \\
0 & 0 & 0 & 1 & 3
\end{array}\right)
$$

Utilizando a técnica de indexação por linhas para $\mathbf{A}$, constrõem-se os vetores sa e ija da seguinte forma

\begin{tabular}{|c||c|c|c|c|c|c|c|c|c|c|c|c|}
\hline índice $k$ & 1 & 2 & 3 & 4 & 5 & 6 & 7 & 8 & 9 & 10 & 11 & 12 \\
\hline ija $|k|$ & 7 & 8 & 9 & 11 & 12 & 13 & 3 & 3 & 2 & 4 & 5 & 4 \\
\hline sa $|k|$ & 2 & 3 & 6 & 8 & 3 & $x$ & 2 & 4 & 5 & 7 & 9 & 1 \\
\hline
\end{tabular}

Desta forma, pode-se armazenar uma matriz esparsa A em apenas dois vetores.

\subsubsection{Aproximação da Equação de Atualização da Velocidade}

$\Lambda$ atualização do campo final de velocidade, é feita pela cquação (3.14). As velocidades na direção $x$ e $y$ são discretizadas como

$$
\begin{gathered}
u^{n+1}=\tilde{u}-\delta \iota c_{1_{p}}, \\
v^{n+1}=\tilde{v}-\delta t c_{2_{p}},
\end{gathered}
$$

\subsubsection{Aproximação das Condições de Contorno}

Para os nós pertencentes a borda do domínio faz-se uma verificação do tipo de condição, "inflow", "outflow" ou "boundary", cm relação a aresta, da seguinte maneira: se uma aresta tem vértices com tipos diferentcs, a velocidade normal e tangencial nesses vértices são nulas, desta forma é necessário que o tamanho para a região de entrada e saída de fluido seja maior que duas arestas (3 vértices).

- Contornos rígidos: No caso em que um nó $v_{i}$ de bordo é tipo "boundary" e têm suas arestas de bordo com nós do tipo "boundary" aplica-se a condição sem escorregamento ("no-slip"), ou seja a componente normal $\left(u_{n}\right)$ e a componente tangencial $\left(u_{t}\right)$ da velocidade do nó $v_{i}$ são nulas.

- Condição de entrada de fluido ("inflow"): No caso em que um nó $v_{i}$ de bordo é tipo "inflow" e têm suas arestas de bordo com nós do tipo "infow", temos $u_{n}=u_{\text {inflow }}$ para a componente normal da velocidade, e $u_{t}=0$ para as componentes tangenciais.

- Condição de saída de fluido ("outflow"): No caso em que um nó $v_{i}$ de bordo é tipo "outflow" têm suas arestas de bordo com nós do tipo "outflow", define-se $\frac{\partial u_{n}}{\partial n}=0$ para a componente normal da vclocidade, e $\frac{\partial u_{t}}{\partial n}=0$ para as componentes tangenciais.

Considera-se uma reta passando pelo vértice $v_{i}$ com direção $m=\left(m_{x}, m_{y}\right)$ onde $m$ é o) vetóor unitário com direção da média dos vetores normais das arestas vizinhas (ver Figura 1.4). Toma-se o ponto $p_{i}$ nesta reta a uma distancia $h$ de $v_{i}$ do lado interior a malha scndo $(x, y)$ as coordenadas de $v_{i}$, as coordenadas de $p_{i}$ serão 


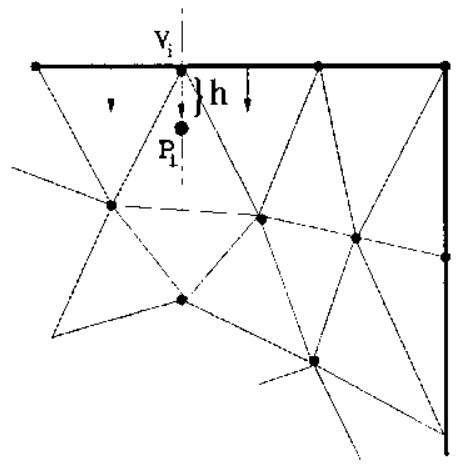

Figura 4.4: Condição de contorno: Outflow

$$
p_{i_{x}} \cdot x-m_{x} h \quad \text { e } \quad p_{i y}=y-m_{y} h
$$

onde $h$ é o valor da menor aresta da malha, então procura-se o triângulo que o novo nó pertence e faz-se uma interpolação linear com os valores das velocidades nos vértices desse triângulo, o valor encontrado será o valor das velocidades do nó $v_{i}$.

\subsection{Método dos Gradientes Bi-conjugados para Sistemas Esparsos}

A aplicação da discretização das equações de Navier-Stokes, pelo método DFG resulia em 1 m sistema linear esparso para o cálculo da equação de Poisson. Na metodologia. GENSM AC, o método gradiente conjugado (GC) é utilizado na resolução da equação de Poisson para a pressão, pois o sistema resultante é esparso, definido positivo e simćtrico. Mas para o método DFG a cquação para a pressão $p$, tem como sistema resultante matriz não simétrica, que por sua ve\%, não permite o uso do método GC. Portanto, a aplicação de $11 \mathrm{~m}$ novo método itcrativo para rasolução de sistemas lineares é necessária.

O objetivo desta seção é apresentar o método dos gradientes bi-conjugados (GBC), para solıção de sistemas lineares

$$
A \cdot \mathbf{x}=\mathbf{b}
$$

com malrì $A$ não simétrica.

A formulação do método GBC, requer que inicialmente, construam-se 4 vetores $\mathbf{r}_{k}, \overline{\mathbf{r}}_{k}$, $\mathbf{p}_{k}$ e $\overline{\mathbf{p}}_{k}, k=1,2, \ldots$ Deve-se fornecer os vetores iniciais $\mathbf{r}_{1}, \overline{\mathbf{r}}_{1}, \mathbf{p}_{1}=\mathbf{r}_{1}$ e $\overline{\mathbf{p}}_{1}=\overline{\mathbf{r}}_{1}$. Desta forma, pode-se definir o seguinte algoritmo

$$
\begin{gathered}
\alpha_{k} \cdots \cdot \frac{\mathbf{r}_{k}^{T} \cdot \overline{\mathbf{r}}_{k}}{\overline{\mathbf{p}}_{k}^{T} \cdot \mathbf{A} \cdot \mathbf{p}_{k}}, \\
\mathbf{r}_{k+1}=\mathbf{r}_{k}-\alpha_{k} \mathbf{A} \cdot \mathbf{p}_{k}, \\
\overline{\mathbf{r}}_{k+1}=\overline{\mathbf{r}}_{k}-\alpha_{k} \mathbf{A}^{T} \cdot \overline{\mathbf{p}}_{k}, \\
\beta_{k}=\frac{\overline{\mathbf{r}}_{k-1}^{T} \cdot \mathbf{r}_{k-1}}{\overline{\mathbf{r}}_{k}^{T} \cdot \mathbf{r}_{k}}, \\
\mathbf{p}_{k+1} \quad \mathbf{r}_{k+1}+\beta_{k} \mathbf{p}_{k}, \\
\overline{\mathbf{p}}_{k+1}=\overline{\mathbf{r}}_{k \mid 1}+\beta_{k} \overline{\mathbf{p}}_{k}
\end{gathered}
$$


Os vetores dessa sequência são bi-orlogonais, o1 seja.

$$
\overline{\mathbf{r}}_{i}^{T} \cdot \mathbf{r}_{j}=\mathbf{r}_{i}^{\prime} \cdot \overline{\mathbf{r}}_{j}=0, \quad j<i,
$$

c também satisfazem a condição

$$
\overline{\mathbf{p}}_{i}^{T} \cdot \mathbf{A} \cdot \mathbf{p}_{j}=\mathbf{p}_{i}^{T} \cdot \mathbf{A}^{T} \cdot \overline{\mathbf{p}}_{j}=0, \quad j<i,
$$

e são reciprocamente ortogonais

$$
\overline{\mathbf{r}}_{i}^{T^{\prime}} \cdot \mathbf{p}_{j}=\mathbf{r}_{i}^{T} \cdot \overline{\mathbf{p}}_{j}=0, \quad j<i
$$

A prova dessas propriedades é apresentada em |Fletcher, 1975|. Portanto, para 11tilizar o algoritmo (4.20), $11 \mathrm{~m}$ vetor inicial $\mathbf{x}_{1}$ é escolhido, e o resíduo inicial define o vetor $\mathbf{r}_{1}$,

$$
\mathbf{r}_{1}=\mathbf{b}-\mathbf{A} \cdot \mathbf{x}_{1}
$$

$\operatorname{com} \overline{\mathbf{r}}_{1}=\mathbf{r}_{1}$. O próximo vetor da sequência de aproximações é calculado por

$$
\mathbf{x}_{k+1,1}=\mathbf{x}_{k} \cdot \alpha_{k} \mathbf{p}_{k}
$$

C'm critério de parada para o método GBC é

$$
\frac{\left|A x_{k+1}-\mathrm{b}\right|}{|\mathrm{b}|}<\text { tol }
$$

onde tol é uma tolerância escolhida de acordo com o problema. Como o método GBC é mais eliciente se a matri\% A for bem-condicionada |Quarteroni et al., 2000|, pode-se utilizar $11 \mathrm{~m}$ método de precondicionamento |Golub and Loan, 1989|, isto é, o sistcma linear (4.19) c transformado para

$$
\left(\tilde{\mathbf{A}}^{-1} \mathbf{A}\right) \mathbf{x}-\tilde{\mathbf{A}}^{-1} \mathbf{b}
$$

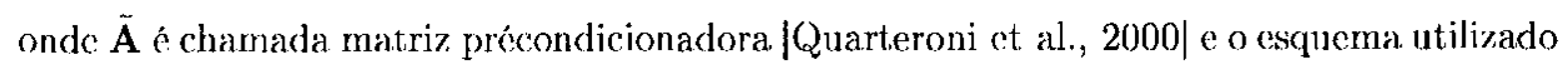
o chamado método dos gradientes bi-conjugado precondicionado (GBCP). Neste trabalho, $\tilde{A}$ foi tomada como a diagonal de A. Para se utilizar um precondicionamento, os vetores $z_{k}$ e $\bar{z}_{k}$ definidos como

$$
\tilde{\mathbf{A}} \mathbf{z}_{k}=\mathbf{r}_{k} \quad \text { e } \quad \tilde{\mathrm{A}}^{T} \overline{\mathbf{z}}_{k}=\overline{\mathbf{r}}_{k}
$$

săo introduxidos no algoritmo (4.20). Note que as equaçōes (4.28) representam sistcmas lineares que devem ser resolvidos a todo passo. Desta forma a matriz $\tilde{A}$ deve ser escolhida de forma que esses sistemas tenham soluçôes simples. Portanto, a definição dos valores $\alpha_{k}$ c $\beta_{k}$ e dos vetores $\mathbf{p}_{k}$ e $\overline{\mathbf{p}}_{k}$ serão modificados para

$$
\begin{aligned}
\alpha_{k} & =\frac{\mathbf{z}_{k}^{T} \cdot \overline{\mathbf{z}}_{k}}{\overline{\mathbf{p}}_{k}^{T} \cdot \mathbf{A} \cdot \mathbf{p}_{k}}, \\
\beta_{k} & =\frac{\overline{\mathbf{r}}_{k+1}^{T} \cdot \mathbf{z}_{k \cdot 1}}{\overline{\mathbf{r}}_{k}^{T} \cdot \mathbf{z}_{k}}, \\
\mathbf{p}_{k+1} & =\mathbf{z}_{k+1}+\beta_{k} \mathbf{p}_{k}, \\
\overline{\mathbf{p}}_{k+1} & \cdots \overline{\mathbf{z}}_{k+1}+\beta_{k} \overline{\mathbf{p}}_{k} .
\end{aligned}
$$


O critério de parada do algoritmo (4.29) para o método GBCP pode ser expresso como

$$
\frac{\left|\tilde{\mathbf{A}}^{-1} \cdot(\mathbf{A x}-\mathbf{b})\right|}{\left|\tilde{\mathbf{A}}^{-1} \cdot \mathbf{b}\right|}<\text { tol. }
$$

Para o método GBC não existem resultados que garantam sua convergência, como no caso do método GC. Desta forma o método GBC pode falhar (mm alguns problemas. Detalhes sobre o método GBC e GBCP podem ser encontrado em |Lanczos, 1952|, |Watson, 1976|, |Vorst, 1992|, |Press ct al., 1992| e |Ayachour, 2003|.

\subsection{Estrutura de dados SHE}

Para garantir uma melhor distribuição dos nós sobre o domínio, é utilizado no método de DFG a estrutura de dados "Singular IIandle-Edge" (SHE), utilizada para a representação de malhas não estruturadas em duas dimensões. A estrutura de dados SHE tem a habilidade de representar vértices singulares e curvas do contorno. A introdução de vértices singulares é comum nos processios de inserção e remoção de triângulos nas malhas não estruturadas. Em aplicaçôes numéricas as condições dos contornos associados é uma equação diferencial que podem ser manipuladas mais facilmente por uma representação explícita dos elementos da fronteira.

\subsubsection{A estrutura "Singular Handle-Edge"}

A singular Handle-Edge é organizada em termos de sete entidades representadas explicitamente, os quais são:

- "sheShell": Esta classe representa cada grupo e triângulos conexos. É nesta entidade onde são armazenadas as listas de cólulas e de componentes de bordo, além de um identificador, número de célılas, número de componentes de bordos e uma referência para a malha. Agrupando os triângulos por componentes conexas, temos que para cada Shell, existe apenas um componente de bordo externo, dentre todos bordos que este pode conter, sendo os outros componentes as bordas dos "buracos" na "Shell".

- "sheVertex": Fsta classe representa cada vértice (ponto ou nó) de uma malha triangular. Nela é armazenada as coordenadas geométricas, a característica de pertencer ao bordo ou não, informações sobre singularidades e identificadores.

- "sheHalfEdge": Esta classe representa cada semi-aresta dos triângulos da malha bidimensional. Nesta entidade é armazenada a informação da cólula a que ela pertence, da aresta vizinha e do vértice pé da semi-aresta. $A$ aresta vizinha pode pertencer a outro triângulo ou ao componente de bordo. As arestas que pertence a dois triângulos, são representadas por dois objctos, uma para cada triângulo. $\Lambda$ s semi-arestas de bordo não têm semi-aresta vizinha.

- "sheSing": Representa cada componente singular incidente a um vértice. Nela é armazenada uma referência para cada aresta de bordo onde o vértice singular é pé. No caso do vértice não ser singular, esta referência será uma aresta qualquer.

- "sheCell": Esta classe representa cada triângulo da malha. As informacoós guardadas por esta classe são as referências para as semi-arestas que pertencem á célıla, uma referência para a malha que contém a célula, c um identificador. 
- "sheBoundaryCp": Representa a(s) componente(s) de bordo de uma malha, cada componente conexa da malha possui sua lista de componentes de bordo ("sheBoundaryCp"), que por sua vez possui um ponteiro para uma lista de "shcBoundary", que são as arestas da referida borda.

- "sheBoundary": Esta classe representa cada aresta de bordo de uma malha triangular. Outra informação armazenada aqui é uma referência à componente de bordo que ela pertence.

As cntidades "sheHalfEdge", "sheSing" e "sheBoundary" são armazenadas em listas circulares dinâmicas, enquanto o resto dos elementos é armazenado em listas não circulares.

a)

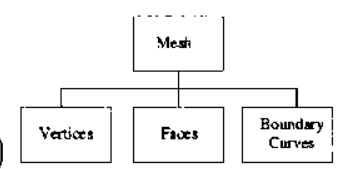

d)

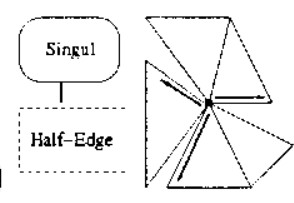

e)

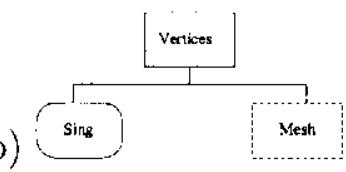

c)
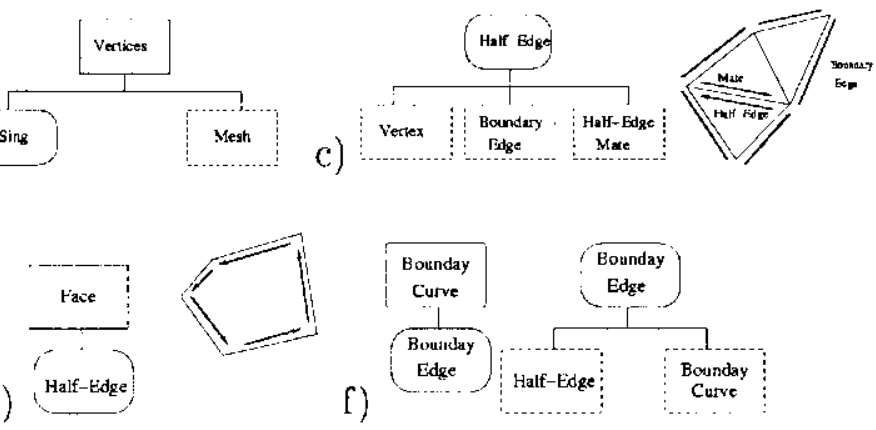

g)

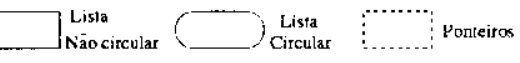

Figura 4.5: Entidades de orgarnização da estrutura de dados SHF.

\subsubsection{Implementação}

$\Lambda$ estrutura de dados SHE e o completo conjunto de métodos para manipular a armazenagem das informaçôs estão implementados em $\mathrm{C}++$.

Um mecanismo chamado iterator foi implementado para percorrer os clementos da SHE, o qual permite a exploração de listas com um simples FOR. Métodos Begin() o End() definidos para cada classe são responsáveis por iniciar e finalizar o iterator. Por exemplo, o seguinte código pode ser cmpregado para imprimir as coordenadas de todos os vértices na malha ao percorrer a lista de Vértice:

Iterator $\langle$ SHE_Vertex $>$ iv;

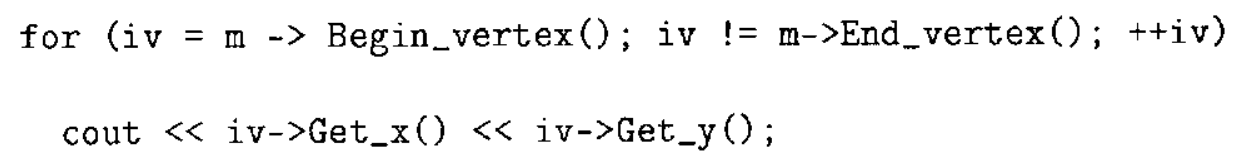

onde m é um ponteiro para o clemento Malha. A principal vantagem do iterator é que ele encapsula as listas e padroniza o acesso a clas. 


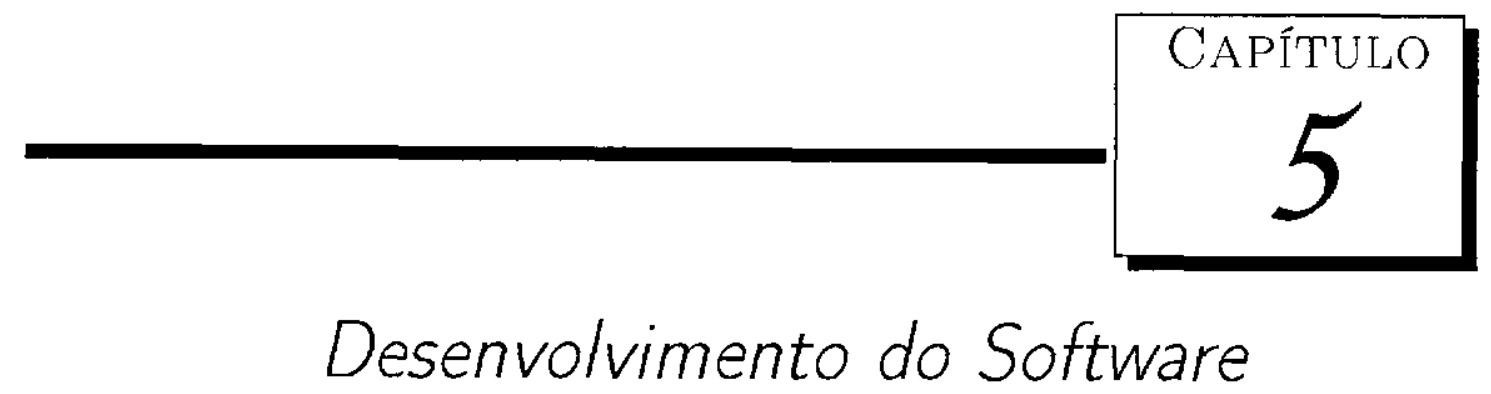

\subsection{Considerações Iniciais}

Para um melhor desenvolvimento do projeto, o estudo de conceitos em engenharia de software (FS) foi incluído visando um melhor desenvolvimento do software, garantindo a aplicaçăo de uma abordagem sistemática, disciplinada e quantificável, para o desenvolvimento, operação e manutenção do softwarc. Com isso o grupo de mecânica dos fluidos, vislumbra extensões e alterações que poderão ser facilitadas no desenvolvimento de novos projetos, como por exemplo a extensão do software para simulação de fluidos Newtonianos generalizados ou discretização das equações, por exemplo, pelo método de volumes finitos.

Foi criado um grupo de estudo entre pesquisadores e doutores em mecânica dos fluidos e engenharia de software para o acompanhamento do desenvolvimento do software, visando uma aplicaçăo adequada para o projeto e um estudo de caso em ES. Em relação à área mecânica dos fluidos, conformo comentado, o cumprimento de atividades de ES para o desenvolvimento de um software "reutilizável". Para o grupo de ES que considera o trabalho, um estudo de caso relevante para a proposta de um modelo para desenvolvimento de software cm ambiente acadêmico |Paiva, 2004].

P'retende-se projetar um software reulilizável, seu projeto deve ser específico para o problema a resolver, mas também genérico para atender fiuturos problemas e requisitos. Deseja-se evilar o re-projeto, ou pelo menos minimizá-lo.

O software está sendo implementado em linguagem $\mathrm{C}++$, utilizando programação orientada a objeto, com UML ("Unified Modeling Language").

Este capítulo apresenta uma introdução dos principais conceitos e definiçōes de ES e o ambiente de similação UmFlow-2D.

\subsection{Engenharia de Software}

A Fingenharia de Software surgiu em meados dos anos 70 numa tentativa de contornar a crise do software e dar um tratamento de engenharia (mais sistemático e controlado) ao 
desenvolvimento de sistemas de software.

Os fundamentos científicos para a ES envolvem o uso de modolos abstratos e rigorosos que permitem ao engenheiro especificar, projetar, implementar o manter sistemas de software, avaliando e garantindo suas qualidades. Além disto, a engenharia de software deve oferecer mecanismos para se planejar e gerenciar o processo de desenvolvimento.

Para o desenvolvimento de um software é necessário

- Instruções que quando cxecutadas produzem a função e o desempenho descjados;

- Fstrutura de Dados que possibilitam que os programas manipulem adequadamente a informação;

- Documentos que descrevam a opcração e o uso dos programas.

\subsection{O Processo de Software}

O Processo de Software abrange um conjunto de três elementos fundamentais para projetar, construir e manter grandes sistcmas de software de forma profissional. São eles:

- Métodos: proporcionam os detalhes de como fazer para construir o software.

Plancjamento e estimativa de projeto

Análise de requisitos de software e de sistemas

Projeto da cstrutura de dados

- Algoritmo de processamento

- Codificaşão

- leste

- Manutenção

- Ferramentas: dão suporte automatizado aos métodos.

- Procedimentos: constituem o elo de ligação entre os métodos e ferramentas

\subsubsection{Modelos de Processos de Software}

Modelos de processes são estratígias de desenvolvimento que abrange camadas de processo, métodos e ferramentas. L'm modelo de software para engenharia de software é escolhido com base na natureza do projeto e da aplicação. nos métodos e ferramentas a serem usados, e nos controles e nos produtos intermediários e finais que são requeridos.

Existem vários modelos de processo de software, cada um representa uma tentativa de colocar ordem em uma atividade caótica. Pode- se citar os seguintes modelos de processo de software:

- O Modelo Sequencial Lincar;

- O Modelo de Prototipação; 
- O Modelo RAD;

- Modelos Fvolutivos de Processo de Software

- O Modelo Incremental

- O Modelo Fspiral

O Modelo de Montagem de Componentes

- O Modelo de Desenvolvimento Concorrente.

\subsection{Padrões de Projeto}

Quando descnvolvemos um projeto, não devemos partir de princípios elementares ou do zero, ao invés disso, devemos reutilizar soluções que funcionaram no passado. Consequientomente encontramos padrões de classes c de comunicação entre objetos, que reaparcem freqüentemente em muitos sistemas orientados a objetos. Os padrões resolvem problemas específicos de projetos e tornam os projetos orientados a objetos mais flexívcis.

"Os padrões de projeto ajudam a escolher alternativas de projeto que tornam um sistema reutilizável e a evitar alternativas que comprometam a reutilização, podem também melhorar a documentação e a manutenção de sistemas ao fornecer uma especificação explícita de interações de classes e objetos e o seu objotivo subjacente. Em suma, ajudam um projetista a obter um projeto "certo" mais rápido" |Gamma et al., 2000|.

O conhecimento de padrões de projeto é importante, nos dá nomes padronizados e definições para as técnicas que usamos.

\subsection{Documento de Requisitos}

O processo de desenvolvimento de softwara compreende um conjunto de atividades que engloba métodos, ferramentas e procedimentos, com o objetivo de produzir softwares que atendam aos requisitos especificados pelos usuários |Mayrhauser, 1990|,|Pressman, 2002|. De acordo com Castro |Castro, 1995| a especificação de requisitos serve como padrão para testar so as fases de projeto e implementação do processo de desenvolvimento de software estão corretas.

A fase de análise de requisitos é fundamental para o processo de desenvolvimento do software, nesta. Casc, devemos especificar as funções e desempenho do software, indicar a interface do software com outros sistemas, estabelecer as restrições de projeto do software |Pressman, 1994|.

O documento de requisitos de um software contém todos os requisitos funcionais e de qualidade do software. $\Lambda$ funcionalidade diz respeito à finalidade a que se propõe o produto do software e é, portanto, a principal característica de qualidade para qualquer tipo de software. Os requisitos de qualidade, também denominados de requisitos näo funcionais inchucm tanto limitaçoes no produto (desempenho, confiabilidade e segurança) como limitações no processo de desenvolvimento (custos, métodos a serem adotados no desenvolvimento e componentes a serem rentilizados). 
Nesta seção, é apresentado um documento de requisitos (informal) do software de simulação numérica de escoamentos de fluidos em malhas não estruturadas.

\subsubsection{Visão Geral do Sistema}

O Software tem como objetivo a simulação de escoamentos de fluidos Newtonianos bidimensionais utilizando malhas não estruturadas. Os usuários deste software, necessitam de resultados numéricos para, por exemplo, simular injeção em moldes com geometria complexa. Fstes resultados são as caractcrísticas (velocidade, pressão) do fluido simulado, que podem ser visualizadas (através de um arquivo de dados) por uma tabela de valores no final da simulação. O sistema de simulação para malhas não estruturadas ć um sistema computacional para modelagem, simulação e visualização de escoamentos de fluidos que possibilita a análise e a observação do comportamento dinàmico de fluidos incompressiveis. Desta forma, esse sistema dividi-se em módılos, e a comunicação deverá ser feita por arquivos.

O sistema de simulação deve ser composto por três módulos:

Modelador: responsável pela modelagem dos dados da simulação;

Simulador: responsável pola implementação das equações que rege o escoamento;

Visualizador: responsável pela visualização dos resıltados obtido pelo simulador.

Fsse documento é responsável pela descrição do módulo simulador, considerando que o módulo modelador está fazendo corretamente sua função.

\subsubsection{Requisitos Funcionais}

\section{B1. Preparação e Manutenção da simulação}

1. O software deve receber informações sobre a simulação.

- nome do domínio

- tempo inicial e final

- ciclo inicial e ciclo final

- espaçamento de tempo para impressão o para gravação automática

- escala de comprimento e velocidade (L e U)

- densidade

- força de gravidade (nos eixos $x$ e $y)\left(g_{y}=g y * 9.81\right)$

- viscosidade

- incremento de tempo inicial

- tolerância para a solução da equação de Poisson

- fatores de controle do passo: Fator, Fatorl e Fator2

- tensão superficial 
- opção de escolha do método para solução das equações de velocidades: DFG, VF, EF

- opção de escolha do tipo de escoamento: Newtoniano, Cross-Model, Power-Law,...

Dependendo do tipo de fluido, variáveis poderão ser acrescentadas. Por exemplo:

- Newtoniano: Nenhuma variável é acrescentada;

- Cross-Model: Zero Viscosity, Infty Viscosity, K(constante), M(Power);

- Power-Law: Zero Viscosity, Infty Viscosity, Density, K(constante), N(Power);

- Bingham: Plastic Viscosity, Yield stress, M(constante);

- SOF-Model: lambda 2, lambda 4, Runge-Kutta order: RK1, RK2, RK3, RK4;

- PTT-Model: Jambda(constante), Epsilon(constante), Xi(constante), Beta(constante).

2. O simulador deve receber o domínio discretizado.

3. O simulador deve receber as variáveis adimensionalizadas: $\mathbf{u}, \mathbf{x}, \mathrm{t}, \mathrm{p}, \mathbf{g}, \rho, \mu$.

4. O simulador deve receber a velocidade inicial normal setada na entrada e saída de fluido.

5. O software deve discretizar as volocidades nos vórtices dos elementos triangulares e a pressão no baricentro dos elementos triangulares.

6. O software deve permitir a escolha da discretização para as cquações governantes. As equações (2.31) e (2.32) devem ser discretizadas utilizando o método DFG como já descrito na seção (4.2).

7. A matriz do sistema (1.6) resultante da aplicação do método mínimos quadrados será resolvido pelo método de eliminação de Gauss.

8. O software deve impor as condiçôes de contorno dadas em (4.2.5).

9. O software deve permitir a escolha do tipo de escoamento.

Somente o modelo Newtoniano está implementado.

10. () software deve permitir a escolha do tipo de célula, sendo que a quantidade de nós de cada célula depende do grau da derivada aproximada. Ver (1.2.1).

\section{B.2 Processamento da simulação}

11. O software deve resolver a equação do Navier-Stokes pelo método GENSMAC.

\section{B.3 Consultas dos Resultados e Emissão de Relatórios}

12. O software deve gerar um arquivo com todos os resultados da simulação que deverá ser lido pelo módulo Visualizador. 


\subsubsection{Diagrama de Caso de Uso}

A medida que os requisitas são elicitados, pode-se criar $11 \mathrm{~m}$ conjunto de cenários que identifica uma linha de uso para o sistema a ser construído. Os cenários, freqü̈entemente chamados de casos de uso, fornecem uma descrição de como o sistema será formado.

Usando a notação UMI, pode-se criar uma representação diagramática de um caso de uso, chamado de diagrama de caso de uso.

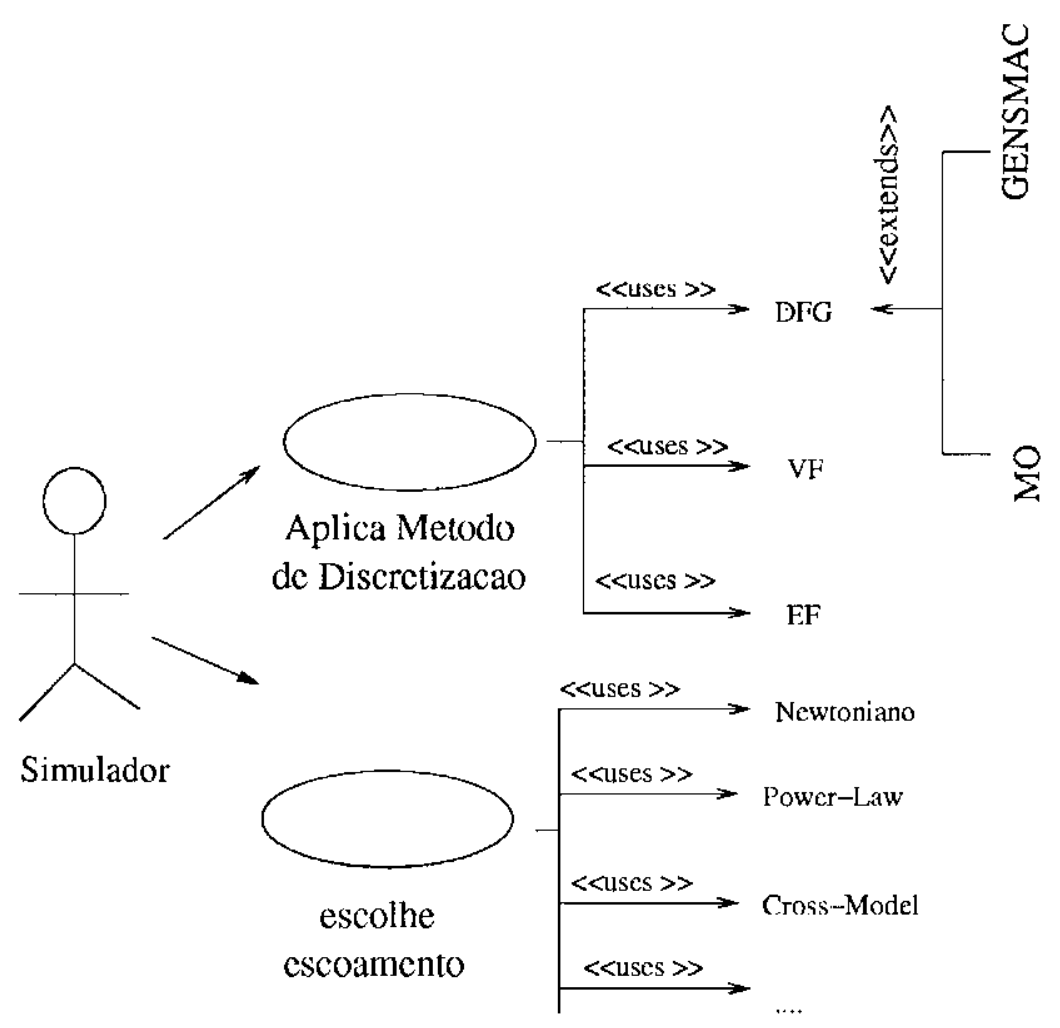

Fignra 5.1: Diagrama de Casos de Uso do software. 


\subsection{Ambiente de Simulação UmFlow-2D}

Neste trabalho, desenvolveu-se os módulos de simulação e re-inicialização do ambiente de simulação UmFlow-2D, baseado no ambiente de simulação FreeFlow-2D, para a simulação de cscoamento de fluidos Newtoniano. A estrutura de dados utilizada foi a estrutıra. de dados SHE. Os módulos modelador e visualizador foram desenvolvidos no projeto de mestrado da aluna Ana Pa.1la Resende Malhciro, sob a orientação do Prof. Dr. Luis Gustavo Nonato, e a interface gráfica dos módulos, no projeto de Iniciação Científica do aluno André Luiz Toyama Carneiro, sob a orientação do Prof. Dr. Luis Gustavo Nonato.

O sistema UmFlow-2D é um sistema computacional para modelagem, simulação e visualização de escoamentos de fluidos que possibilita a análise e a observação do comportamento dinâmico de fluidos incompressiveis. Desta forma, esse sistema dividi-se em módulos, e a comunicação ć feita por arquivos.. A seguir apresenta-se um resumo sobre esses módulos.

\subsubsection{Modelador}

O modelador bidimensional é um editor gráfico interativo que permite a definição dos objetos bidimensionais do escoamento de fluidos, que inclui os dados que configiram o escoamento. Fsses dados são: domínio, tempo inicial e final, ciclo inicial e final, intervalo de tempo para impressăo e gravação automática, escalas de comprimento e velocidade, força de gravidade, densidade, viscosidade, incremento de tempo inicial, tolerância para resolução dos sistemas lineares, fatores de controle no tempo, tipo de fluido para o escoamento e opçóes do método de resolução: fluido Newtoniano e método de Diferenças Finitas Generalizadas.

Através da interface de entradas de dados, o usuário pode modificar os campos desejados e salvar a configuração em $11 \mathrm{~m}$ arquivo.

A entrada dos dados do domínio e de todos os dados necessários para a simulação é obtidos durante a modelagem do problema. O modelador cria três arquivos de saída, que serão os arquivos de cntrada para o simulador.

\subsubsection{Simulador}

O simulador consiste em $11 \mathrm{~m}$ conjunto de programas baseado na metodologia GFNSMAC, cuja finalidade é resolver problemas de escoamentos transientes de fluidos incompressiveis. Nesse módulo, que não tem interface gráfica, as equações de Navier-Stokes c a equação da continnidade são resolvidas passo a passo como foi discutido na seção 3.6 pelo método de diferenças finitas generalizadas.

O simulador gera os resultados em arquivos que são lidos pelo módulo visualizador.

\subsubsection{Visualizador}

Lsse módılo é responsável pela visualiza(ũo gráfica dos resultados da simulação de escoamentos de fluidos.

\subsubsection{Re-inicializador}

O módulo re-inicializador é responsável em reiniciar a simulação do ponto em q11e parou $011 \mathrm{em}$ realizar modificaşões em algum campo para novas simulações. No caso de interrupção acidental pode-se utilizar o re-inicializador: que tem como dados de cntrada o ciclo final, tempo final, intervalo de tempo para impressão e gravação, tolerância para o método que 
resolve os sistemas lineares envolvidos na simulação e os fatores controladores do tempo. 


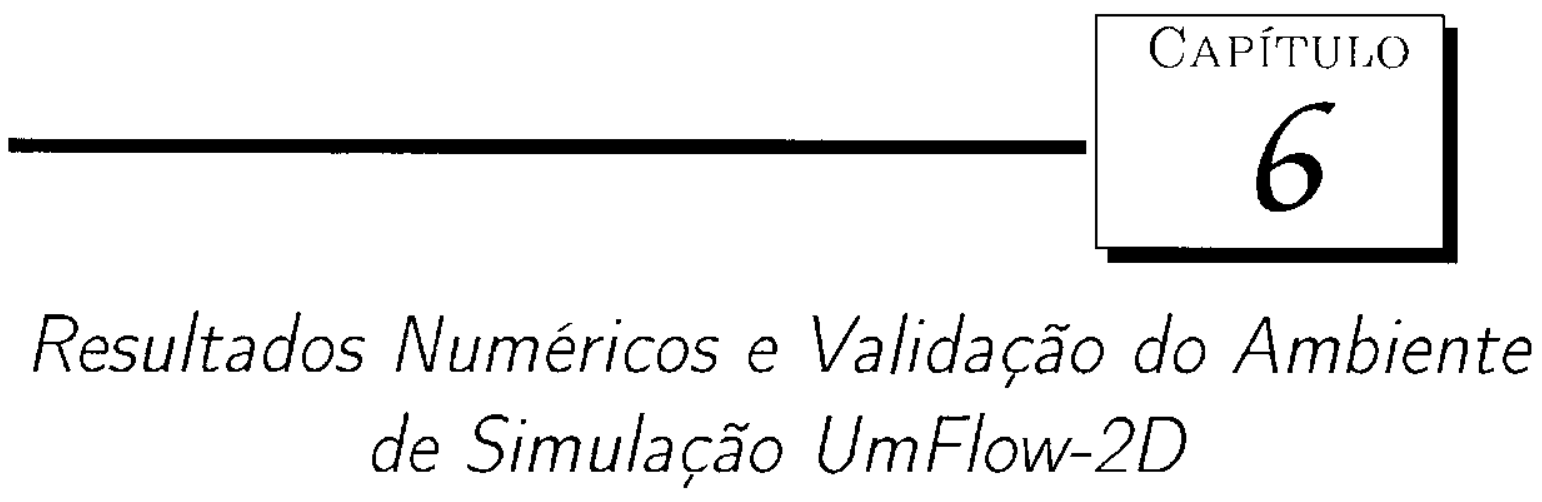

\subsection{Introdução}

Neste capítulo, são apresentadas simulações para validação do software o simulaçós es de escoamentos de fluidos Newtonianos incompressíveis para problemas bidimensionais na presença de contornos rígidos.

Em particular, os métodos numéricos foram aplicados aos seguintes problemas: escoamento em um canal e simulação numa expansão brusca para validar as técnicas numéricas, simulação de uma placa com contração e simulação em domínio com geometria curva.

O ambiente de simulação é o UmFlow-2D, desenvolvido neste trabalho descrito no capítulo 5. Todas as simulaçöes numéricas neste trabalho foram realizadas em microcomputadores

- Dual Athlon XP MP 2200, 1.0 Gb de memória RAM, 80.0 Gb de disco rígido e sistema operacional Linux Slackware 9.0, encontrados no Laboratório de Computação de Alto Desempenho (ICAD) do ICMC-LSP.

\subsection{Simulação do Escoamento em um Canal}

Para o estudo da validação dos resultados numéricos fornecido pelo software de simulação, considera-se o escoamento de um fluido entre duas placas paralelas, conhecido como escoamento de Ilagen-Poiseuille (ver figura 6.1).

O escoamento de Hagen-Poiseuille, estudado por Hagen em 1839 e por Poiseuille em 1840, é muito importante pois ele é um dos poucos problemas para as equaç̃es de Navier-Stokes para o qual uma solução analítica existe, possibilitando a validação do código.

A solução analítica para este problema scrá brevemente discutida, podendo ser encontrada $\mathrm{cm}$ |Batchelor, 1970|. Fm resumo, de acordo com Batchelor, a velocidade $u$ na direçáo 


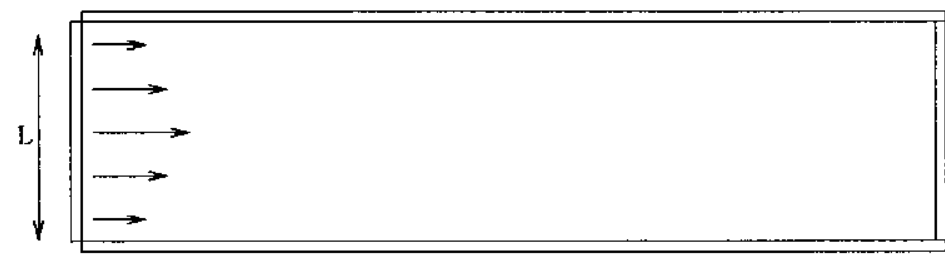

Figura 6.1: Domínio para um escoamento de Hagen-Poiseuille. O escoamento ć da esqucrda para a direita e o comprimento do canal ó $3 L$.

do escoamento e a uma distância $y$ da parede inforior do canal é dada pela expressão

$$
u(y)=-\frac{1}{2 \mu} \frac{\partial p}{\partial x}\left(y L-y^{2}\right)
$$

onde $\mu$ é o coeficiente de viscosidade. O gradiente de pressão é dado pela expressão

$$
\frac{\partial p}{\partial x}=-12 \frac{\mu Q}{L^{3}}
$$

c a vazão $Q$ é definida como

$$
Q=\int_{0}^{L} u(y) d y
$$

Para obter a solução analítica (6.1) da velocidade em função do gradiente de pressão deve-se definir um perfil de vclocidade. Desta forma defini-se $u$ como uma velocidade normal à fronteira de entrada de fluido e $U$ uma velocidade de referência. Quando

$$
u(y)=U\left(1-\frac{y^{2}}{L^{2}}\right)
$$

o perfil de velocidade é do tipo parabólico, e quando

$$
u(y)=U,
$$

defini-se o perfil de velocidade do tipo reto. $\Lambda$ prescrição de um perfil de velocidade é fundamental, pois muitas vezes, não é possível conhecer a distribuição de velocidades através de uma fronteira de fluido, sendo conhecida apcnas a vazão ou a descarga. Desta forma, a solucũo ó prescrever um perfil de velocidade arbitrário, mas compatível com o problema tratado. Após determinar o perfil de velocidade, pode-se calcular a solução analítica para o escoamento de Hagen-Poiscuille. Para isto, é necessário fixar o valor de $L$ e da velocidade de referência $U$.

A vazão $Q$ para o perfil parabólico é dada pela expressão

$$
Q \quad \int_{0}^{L} u(y) d y \quad \int_{0}^{L} U\left(1-\frac{y^{2}}{L^{2}}\right) d y
$$

e para o perfil reto,

$$
Q=\int_{0}^{L} u(y) d y=\int_{0}^{L} U d y
$$


Escolhendo $L=1$ e $U=1$, a vazão $Q$ é calculada diretamente, sendo possível então, determinar o valor do gradiente de pressão (6.2), ou seja

$$
\frac{\partial p}{\partial x}=-8 \mu, \text { para o perfil parabólico, }
$$

$\mathrm{e}$

$$
\frac{\partial p}{\partial x}=-12 \mu, \text { para o perfil reto. }
$$

Portanto, substituindo a equação (6.8) em (6.1) obtêm-se a solução analítica para o perfil parabólico, na forma.

$$
u(y)=-4 y(y-1)
$$

e no caso do perfil reto, ao substituir (6.9) em (6.1) tem-se

$$
u(y)=-6 y(y-1) \text {. }
$$

Como exemplo para a validação do sofware de simulação deste trabalho, considere duas placas paralelas separadas a pela distância $L=1$, formando um canal, que no início da simulação está completamente cheio com uma velocidade constante igual a $u=1.0 \mathrm{~ms}^{-1}$ e fluido é injetado na entrada do canal à uma velocidade prescrita. Quando atinge-se o estado estacionário, os valores das velocidades ao longo do canal deve ser comparados com a solução analítica. O desenvolvimento do fluido dentro do canal, e as comparações podem ser realizadas, a partir de um modelo para simulação.

Para verificarmos este fato e compararmos os resultados numéricos com a solução dada pelas equações (3.36) e (3.35), a simulação foi realizada utilizando três malhas.

- malha 1 (grossa): 183 células; $\delta h \simeq 0.2$;

- malha 2 (intermediária): 728 células; $\delta h \simeq 0.1$;

- malha 3 (fina): 2853 células; $\delta h \simeq 0.005$;

As malhas não estruturadas triangulares geradas sobre o canal nas quais foram feitos os cálculos da solução do problema foi gerada pelo Easymesh |Niceno, 2001|.

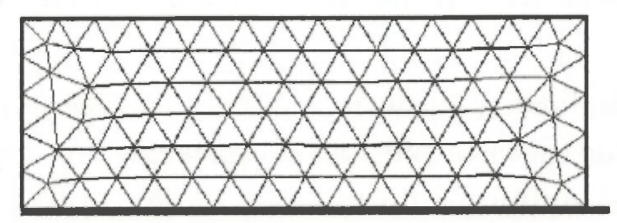

Figura 6.2: Malha com 183 elementos, gerada pclo Easymesh. 


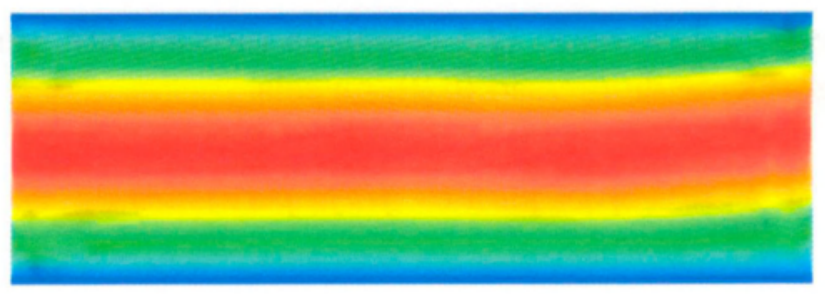

Velocidade $(\mathrm{m} / \mathrm{s})$

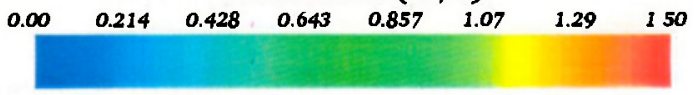

Figura 6.3: Simulação numérica do escoamento de Hagen-Poiseuille com perfil reto: campo de velocidade na direção $\mathrm{x}$, calculados na malha intermediária .

O modelo para esse escoamento é descrito a seguir:

- Dimensão do domínio: $3.0 \mathrm{~m} \times 1.0 \mathrm{~m}$;

- Diâmetro do injetor $(s): 1.0 \mathrm{~m}$;

- Viscosidade : $1.00 \mathrm{Ns} \mathrm{m}^{-2}$;

- Densidade : $1.0 \mathrm{~kg} \mathrm{~m} \mathrm{~m}^{-3}$;

- Parâmetros de escala: $L=1.0 \mathrm{~m}$ e $U=1.0 \mathrm{~ms}^{-1}$;

- Número de Reynolds $(R e=L U / \nu): 1$;

- Número de Froude $(F r=U / \sqrt{g} L): 0.319275$.

Considera-se na simulação do escoamento de Hagen-Poiseuille, injetação de fluido na entrada do canal à uma velocidade prescrita e com perfil do tipo reto. A condição para escoamento totalmente desenvolvido imposta na entrada de fluido é dada pela equação (6.11).

Quando atinge-se o estado estacionário, os valores das velocidades ao longo do canal devem ser comparadas com a solução analítica dada pela expressão (6.11). Após a descrição da solução analítica e o desenvolvimento do fluido dentro do canal, as comparações podem ser realizadas.

A figura (6.4) mostra os resultados numéricos obtidos no escoamento sobre as três malhas não estruturadas. A região considerada para a comparação dos resultados numéricos com a solução analítica, para as três malhas, é no meio do canal, após o escoamento encontrar-se em estado estacionário.

Como pode-se observar, os resultados numéricos são bastante semelhante à solução analítica, ou seja, os valores numéricos obtidos, sobre as três malhas, estão em boa concordância com a solução analítica e mostrando convergência de acordo com o refinamento da malha.

Para validação do escoamento no canal considera-se também injetação de fluido na entrada do canal à uma velocidade prescrita e com perfil do tipo parabólico. A condição para escoamento totalmente desenvolvido imposta na entrada de fluido é dada pela equação 


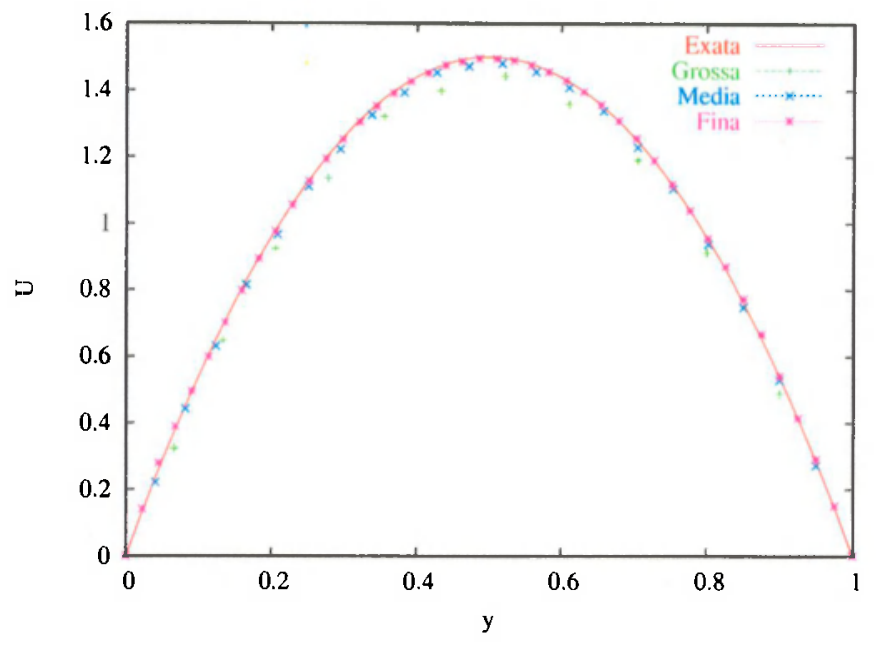

Figura 6.4: Comparação entre soluções numéricas obtida pelo ambiente de simulação UmFlow-2D e a solução analítica dada pela equação (6.11), sobre as três malhas, com $R e=1$.

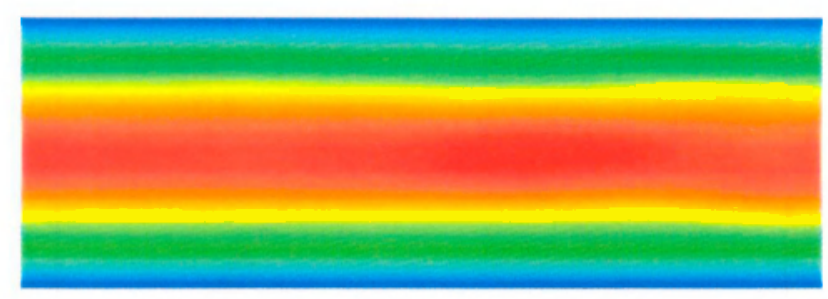

Velocidade $(\mathrm{m} / \mathrm{s})$

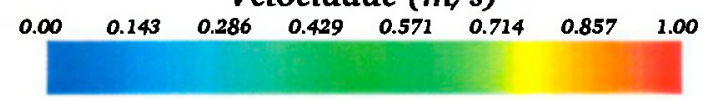

Figura 6.5: Simulação numérica do escoamento de Hagen-Poiseuille com perfil de parabólico: campo de velocidade na direção $\mathrm{x}$, calculados na malha fina.

(6.10). Quando atinge-se o estado estacionário, os valores das velocidades ao longo do canal são os mesmos que os impostos na entrada de fluido, e desta forma, pode-se comparar os resultados numéricos com a solução analítica dada pela expressão (6.10).

Como pode-se observar pela figura (6.6), os resultados numéricos também são bastante semelhante à solução analítica, como no problema anterior, os valores numéricos obtidos, sobre as três malhas, estão em boa concordância com a solução analítica mostrando convergência de acordo com o refinamento da malha.

A simulação do escoamento de Hagen-Poiseuille com perfil parabólico utilizando-se os números de Reynolds 10 e 100, também faz parte da validação do simulador. E considerado no modelo, viscosidade: $0.10 \mathrm{Ns} \mathrm{m}^{-2}$ para $R e=10$ e viscosidade: $0.010 \mathrm{Ns} \mathrm{m}^{-2}$ para $R e=100$.

A figura (6.7) mostra os resultados numéricos obtidos no escoamento sobre uma malha não estruturada com 4.426 elementos triangulares $(\delta h \simeq 0,004 \mathrm{~m})$, para diferentes valores do número de Reynolds. A região considerada para a comparação dos resultados numéricos com a solução analítica, para as três simulações é no meio do canal, após o escoamento encontrar-se em estado estacionário. 


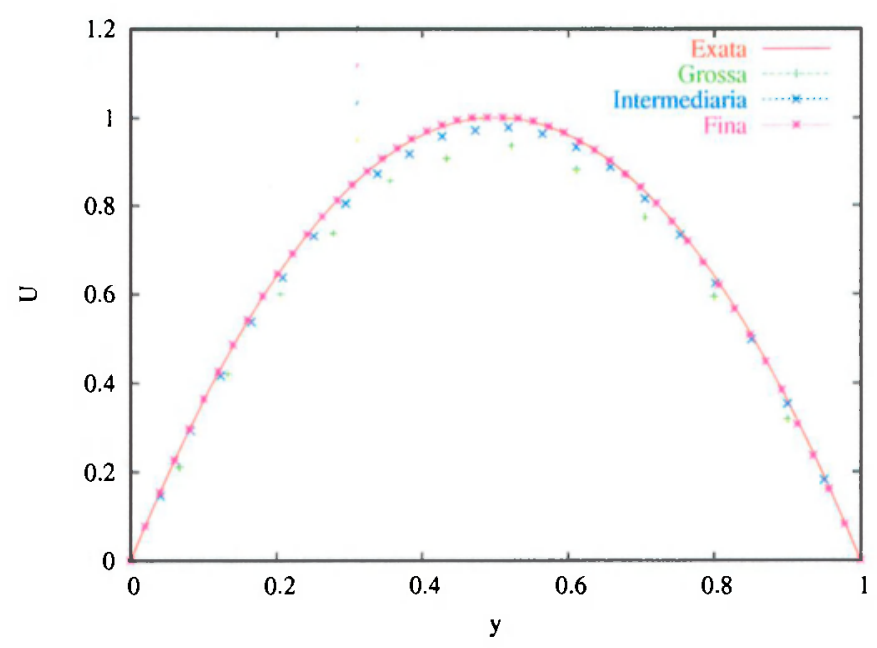

Figura 6.6: Comparação entre soluções numéricas obtida pelo ambiente de simulação UmFlow-2D e a solução analítica dada pela equação (6.11) , sobre as três malhas, com $R e=1$.

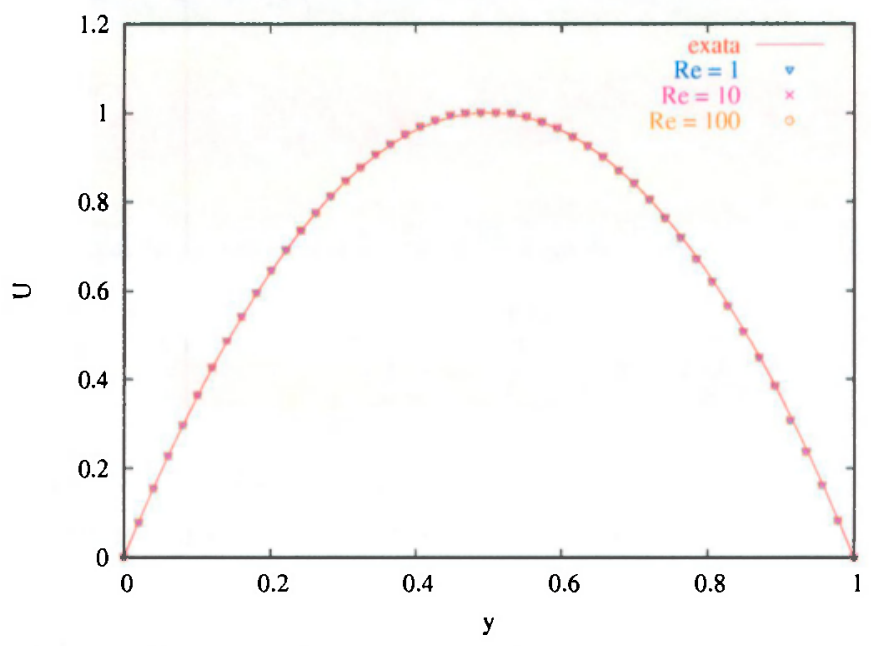

Figura 6.7: Comparação entre soluções numéricas obtida pelo ambiente de simulação UmFlow-2D e a solução analítica dada pela equação (6.11), sobre uma malha (4.426 elementos triangulares), para $R e=1, R e=10$ e $R e=100$.

Como pode-se observar pela figura (6.7), os resultados numéricos das três simulações estão muito semelhantes e de acordo com a solução analítica, ou seja, os valores numéricos obtidos, sobre as malhas, com diferentes valores de Reynolds, estão em boa concordância com a solução analítica e mostrando a invariância com relação ao número de Reynolds.

\subsection{Simulação numa Expansão Brusca}

Para o problema bidimensional numa expansão brusca [Stuart and Dochan, 1991] foi realizada simulação .

A geometria do problema é mostrada na figura (6.8), em que a altura da expansão brusca $s$ é metade da largura do canal. 
$\mathrm{Na}$ entrada do canal, o perfil de velocidade é parabólico, isto é,

$$
u(\ddot{y})--\frac{4 U}{s^{2}}\left(\ddot{y}-\frac{s}{2}\right)^{2}+U
$$

em que $U$ é a escala de velocidade e $s$ o comprimento da seção de entrada. O contorno de saída de fluido está localizado a $30 s$ do degrau. O número de Reynolds para esse problema é calculado por

em que

$$
R e=\frac{2 s \bar{v}}{\nu}
$$

$$
v=\frac{1}{l} \int_{0}^{i} u(y) d y
$$

é a velocidade média na entrada do canal. Calculando-se a integral (6.14) obtém-se

$$
R e=\frac{2}{3} U \frac{2 s}{\nu}
$$

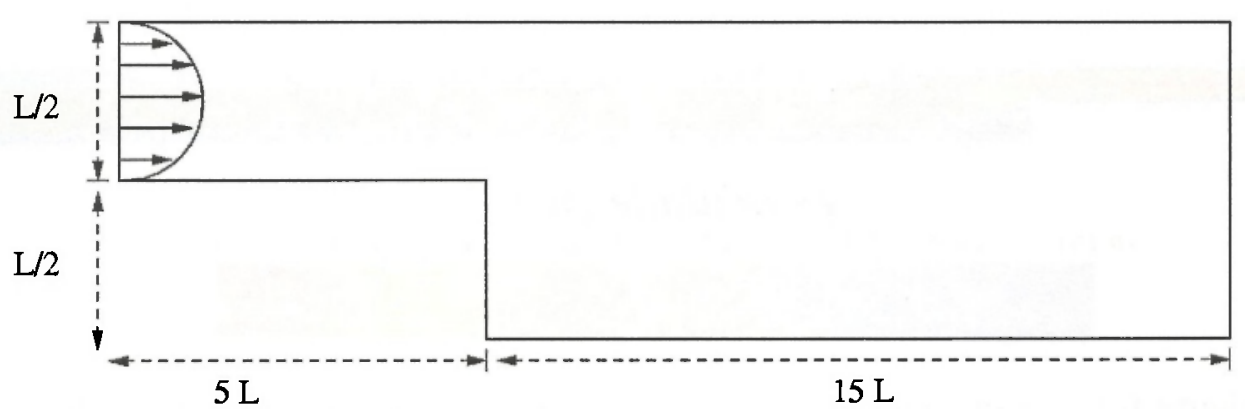

Figura 6.8: Geometria do problema da expansão brusca.

Para uma simulação do problema em questão, o seguinte modelo foi empregado:

- Dimensão do domínio: $2,0 \mathrm{~m} \times 0,1 \mathrm{~m}$;

- Diâmetro do injetor $(s): 0,05 \mathrm{~m}$;

- Parâmetros de escala: $L=0,1 \mathrm{~m}$ e $U=0,10 \mathrm{~ms}^{-1}$;

- Número de Reynolds: 1.0 e 100.

A simulação realizada com o UmFlow-2D, utilizou uma malha com 4.214 elementos triangulares $(\delta h \simeq 0,01 \mathrm{~m})$. Essa simulação é mostrada na figura (6.9). A figura (6.10) apresenta a simulação obtida com o FreeFlow-2D.

As duas simulações apresentam campos de velocidades bastante próximos. Pode-se observar que ambas apresentam uma recirculação na região do degrau.

Esse problema foi bastante estudado e simulado utilizando o FreeFlow-2D na dissertação de Brandi |Brandi, 2005|, onde comparou-se os resultados de outros autores.

A simulação para $R e=100$, obtida com o FreeFlow-2D, é mostrada na figura (6.11)

Pela figura (6.11) podemos observar um aumento da recirculação com o aumento do número de Reynolds. 
$\underset{0.283}{\text { Velocidade }}(\mathrm{m} / \mathrm{s})$

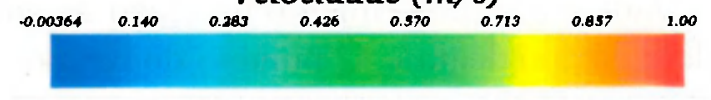

Figura 6.9: Problema da expansão: campo de velocidade na direção x.

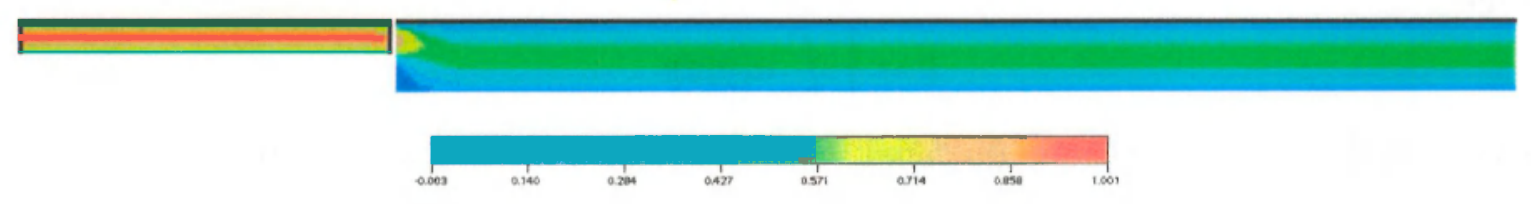

Figura 6.10: Problema da expansão: campo de velocidade na direção $\mathrm{x}$.

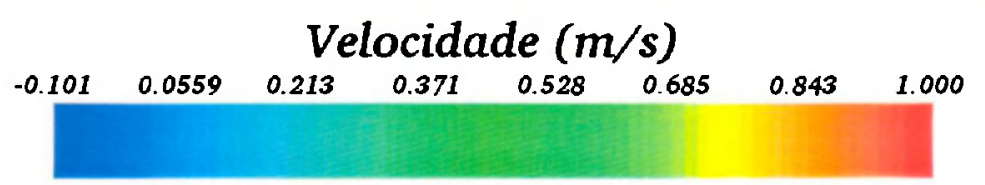

Figura 6.11: Problema da expansão: campo de velocidade na direção x.

\subsection{Simulação de uma Placa com Contração}

O problema da contração carateriza-se pelo escoamento de um fluido através de um canal de diâmentro $L$ que passa a escoar em um canal de diâmetro menor $L / 4$. O domínio do escoamento para o problema é apresentado na figura (6.12)

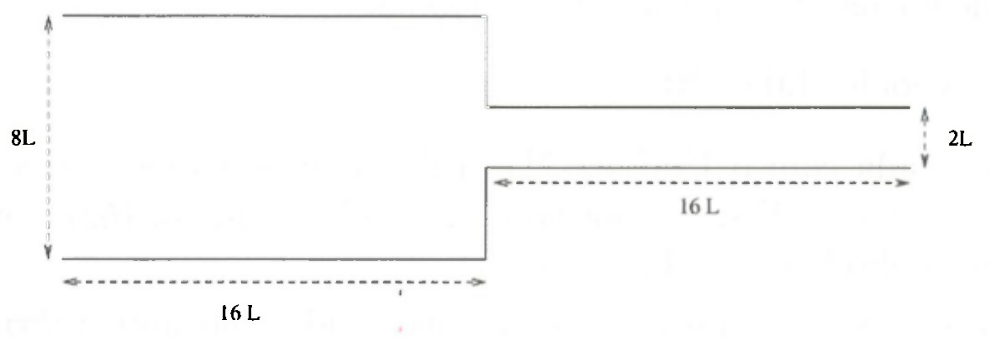

Figura 6.12: Placa retangular com contração.

No início da simulação o domínio está completamente cheio com uma velocidade constante igual a $u=1.0 \mathrm{~ms}^{-1}$ e fluido é injetado na entrada do canal com um perfil de velocidade do tipo parabólico

Para uma simulação do problema em questão, o seguinte modelo foi empregado: 
- Comprimento das cavidades: $L_{1}=8.0 \mathrm{~cm}$ e $L_{2}=8.0 \mathrm{~cm}$;

- Diâmetros das cavidades: $D_{1}=8.0 \mathrm{~cm}$ e $\bar{\nu}_{2}=2.0 \mathrm{~cm}$;

- Parâmetros de escala: Uscale $=1,0 \mathrm{~ms}^{-1}$ Lscale $=1,0 \mathrm{~m}$;

- Número de Reynolds $(R e): 10$ e 100.

A simulação realizada com o UmFlow-2D, utilizou uma malha com 5.681 elementos triangulares $(\delta h \simeq 0,17 \mathrm{~m})$. As simulações é mostrada nas figuras (6.13)- (6.16).
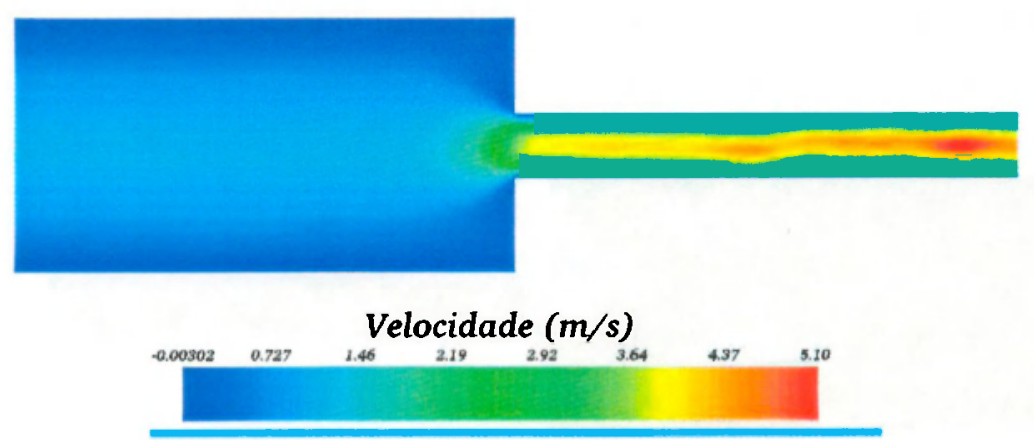

Figura 6.13: Placa retangular com contração: campo de velocidade na direção $\mathrm{x}, \mathrm{Re}=10$.

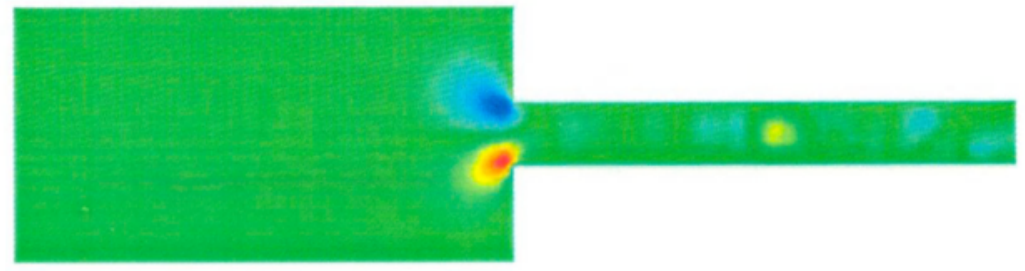

\section{Velocidade $(\mathrm{m} / \mathrm{s})$}

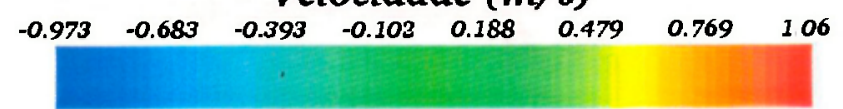

Figura 6.14: Placa retangular com contração: campo de velocidade na direção y, $\operatorname{Re}-10$. 

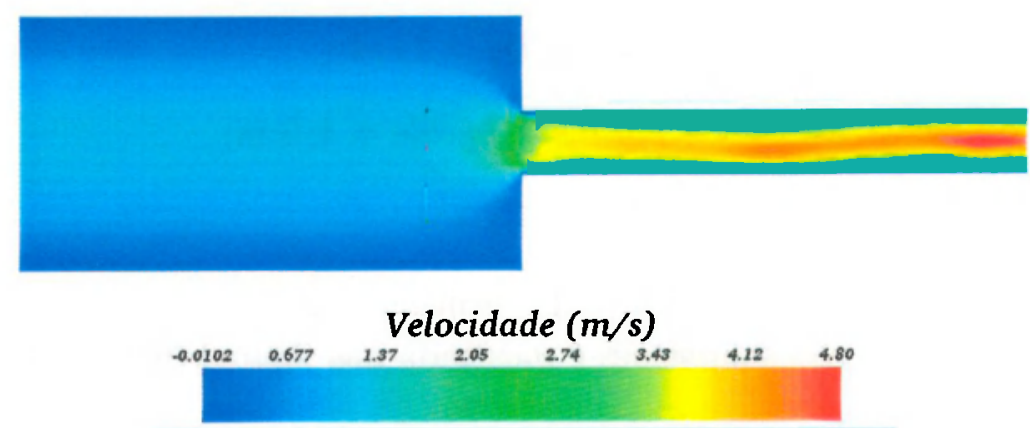

Figura 6.15: Placa retangular com contração: campo de velocidade na direção $\mathrm{x}, \mathrm{Re}=100$.

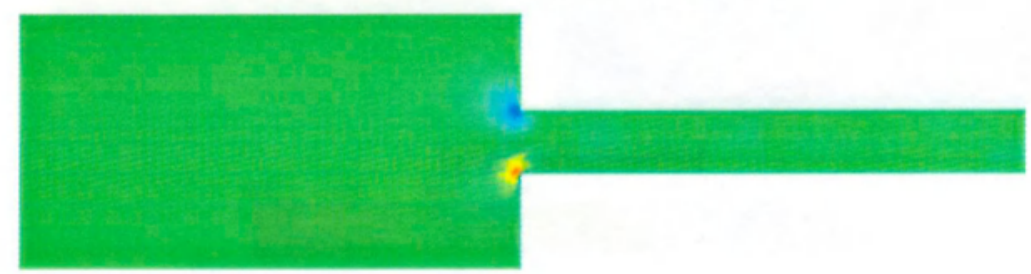

Velocidade $(\mathrm{m} / \mathrm{s})$

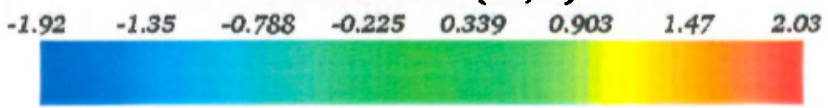

Figura 6.16: Placa retangular com contração: campo de velocidade na direção y, $\operatorname{Re}=100$.

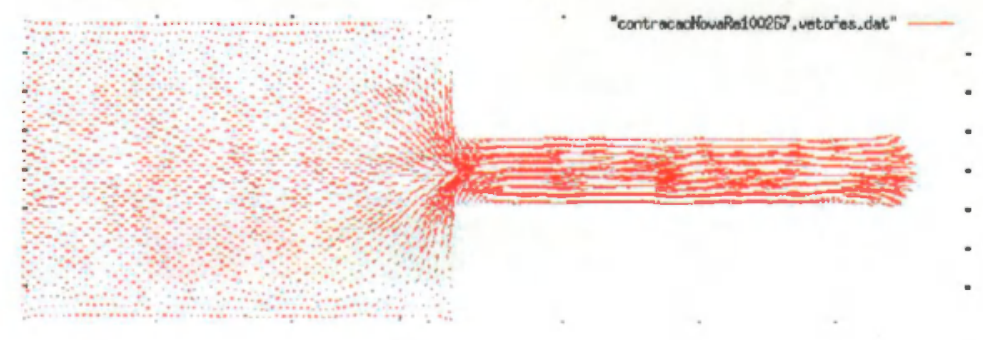

Figura 6.17: Placa retangular com contração: vetores velocidade, Re -100 .

Pode-se observar nas regiões dos cantos superior e inferior direito da primeira cavidade uma recirculação, sendo estás maiores para $R e=100$ do que para $R e=10$.

\subsection{Simulação em domínio com Geometria Curva}

Esta seção apresenta a simulação numérica de um problema bidimensional num domínio com geometria curva. A figura (6.18) mostra o domnio.

No início da simulação o domínio esta completamente cheio com uma velocidade constante igual a $u=1.0 \mathrm{~ms}^{-1}$ e fluido é injetado na entrada do canal à uma velocidade 


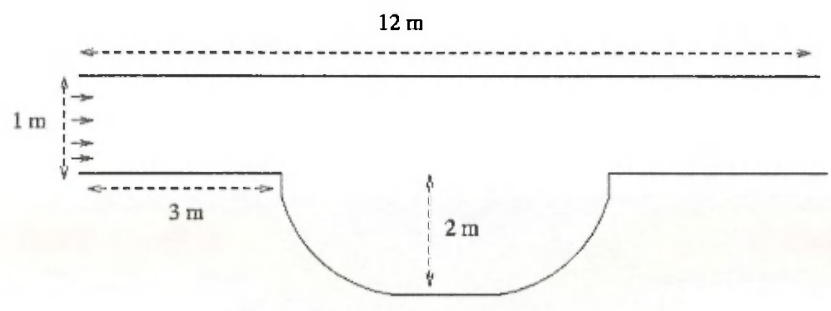

Figura 6.18: Geometria do problema com geometria curva.

prescrita.

Para uma simulação do problema em questão, o seguinte modelo foi empregado:

- Dimensão do domínio: $1,0 \mathrm{~m} \times 12 \mathrm{~m}$;

- Diâmetro do injetor $(s): 1 \mathrm{~m}$;

- Velocidade de injeção $(U): 1,0 \mathrm{~ms}^{-1}$;

- Parâmetros de escala: Uscale $=1,0 \mathrm{~ms}^{-1}$ Lscale $=1,0 \mathrm{~m}$;

- Número de Reynolds (Re): 1.0 e 100.

A simulação realizada com o UmFlow-2D, utilizou uma malha com 4.845 elementos triangulares $(\delta h \simeq 0,10 \mathrm{~m})$. Essas simulações é mostrada nas figuras (6.20)- (6.23).

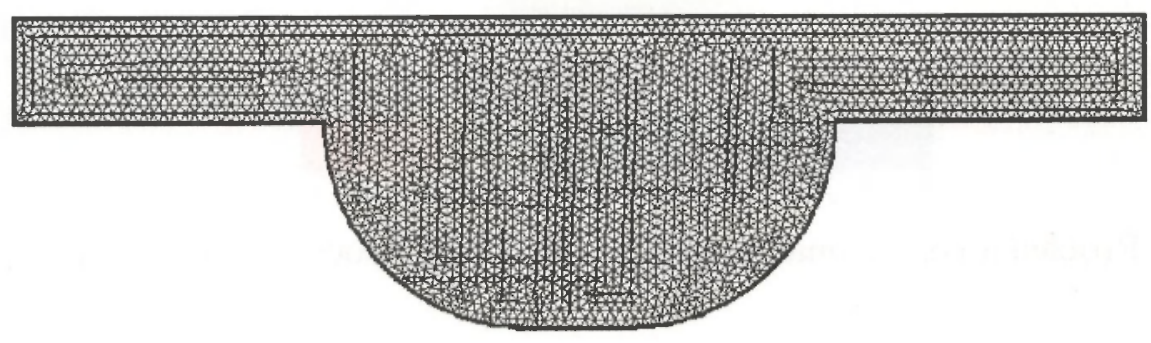

Figura 6.19: Malha com 4.845 elementos, gerada pelo Easymesh.

Através das figuras, observa-se uma recirculação na região curva em menor escala para $R e=1$ e em maior escala para $R e=100$, o que era esperado. 


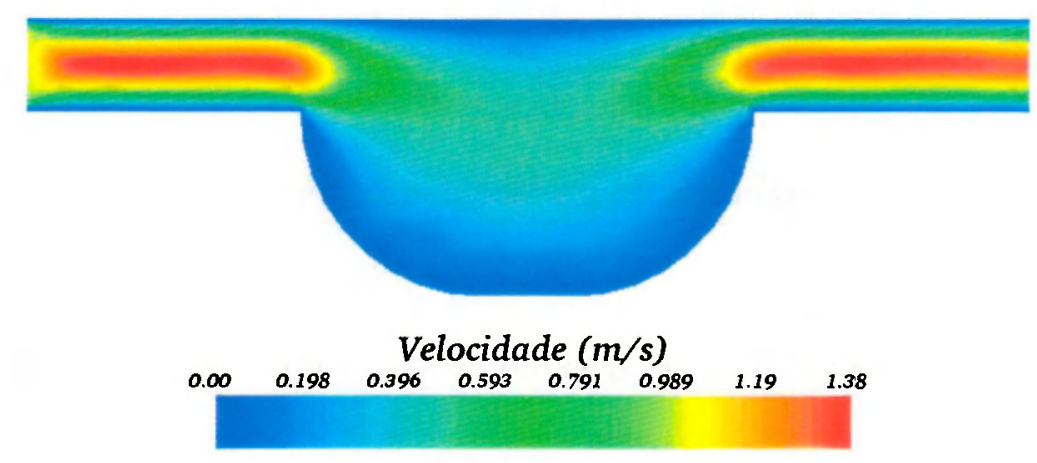

Figura 6.20: Problema com geometria curva: campo de velocidade na direção $\mathrm{x}, \mathrm{Re}=1$.

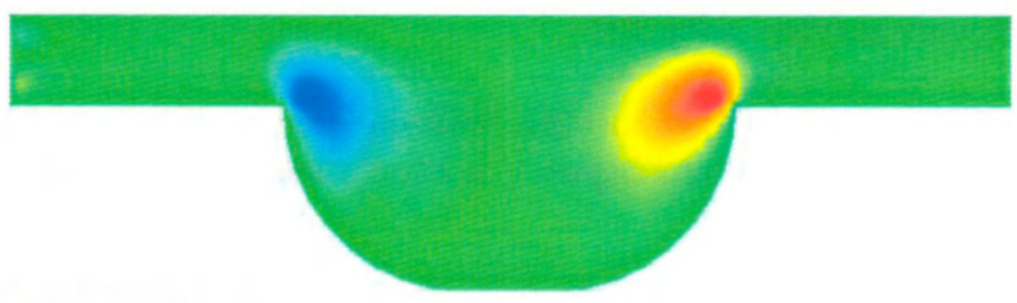

Velocidade $(\mathrm{m} / \mathrm{s})$

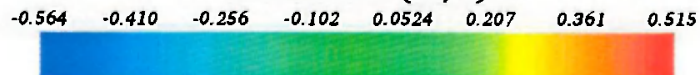

Figura 6.21: Problema com geometria curva: campo de velocidade na direção y, $\operatorname{Re}=1$.

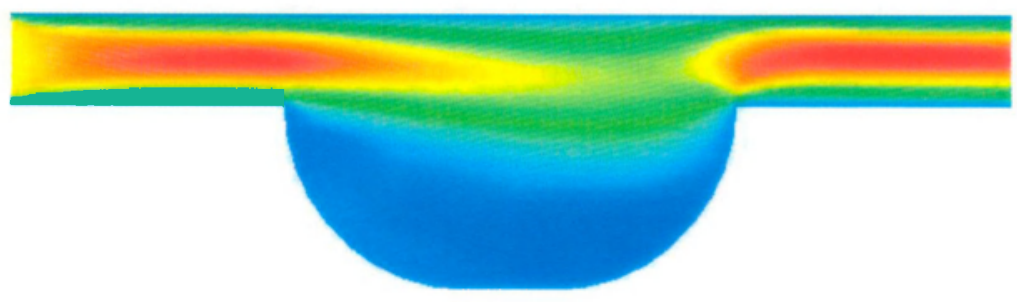

Velocidade $(\mathrm{m} / \mathrm{s})$

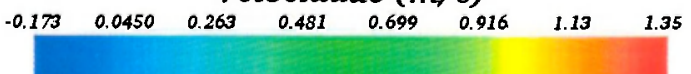

Figura 6.22: Problema com geometria curva: campo de velocidade na direção $\mathrm{x}, \mathrm{Re}-100$. 


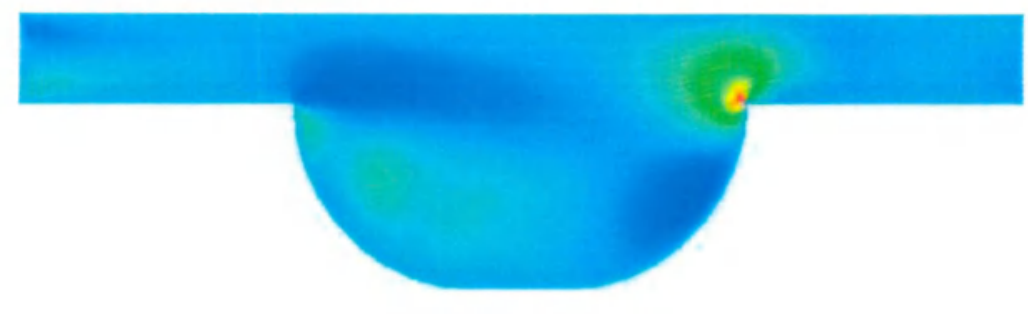

Velocidade $(\mathrm{m} / \mathrm{s})$

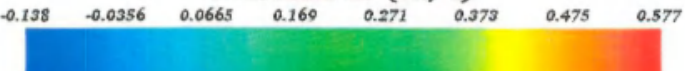

Figura 6.23: Problema com geometria curva: campo de velocidade na direção y, Re $=100$.

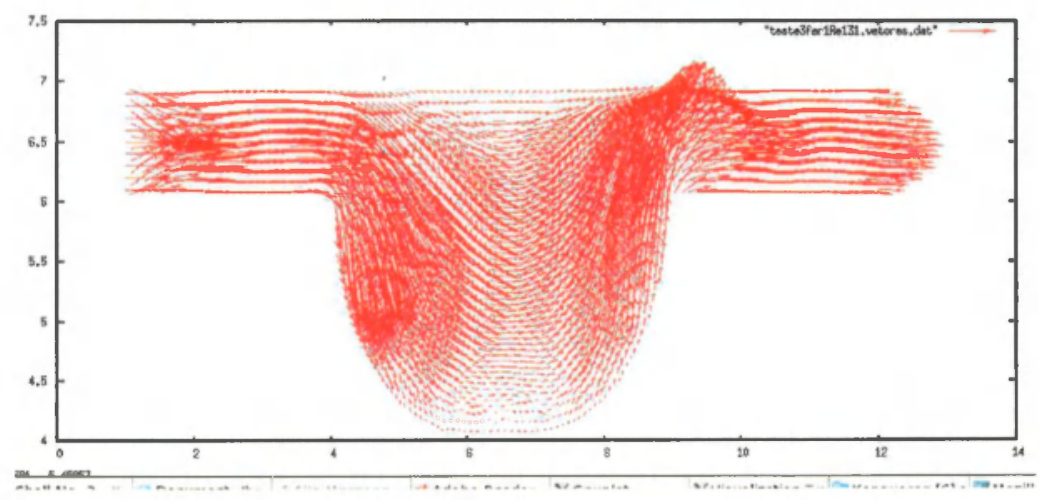

Figura 6.24: Problema com geometria curva: vetores velocidade, $\operatorname{Re}-1$.

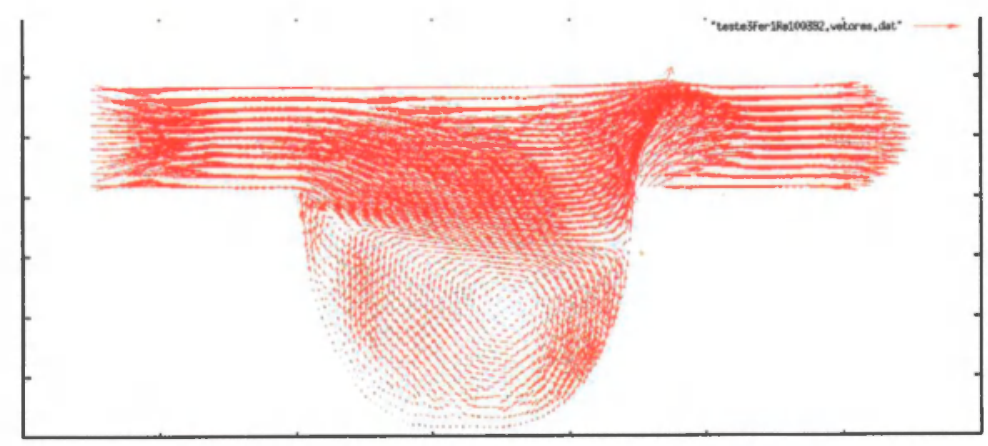

Figura 6.25: Problema com geometria curva: vetores velocidade, $\operatorname{Re}-100$. 


\section{Considerações Finais}

O objetivo desse trabalho de mestrado é o desenvolvimento de um software de simulação, que é parte de um sistema de simulação integrado para escoamento de fluido incompressível bidimensional em malhas não estruturadas denominado UmFlow-2D. Com o desenvolvimento desse ambiente de simulação, dá-se início uma nova etapa de simulações, sendo agora possivel em domínios complexos que eventualmente so aproxima mais dos objotos reais.

Para a implementação do módulo de simulação foram utilizados o método de diferenças finitas generalizadas e uma estrutura de dados topológica SlHE. Para a solıção das equações governantes, foram utilizades o método semi-lagrangeano e o método da projeção. O ambiente de simulação UmFlow-2D foi validado considerando-se o problema de escoamento de fluido em um canal e foi realizada sirnulação de escoamento de um fluido numa expansão brusca. O primeiro problema aparece com frequência na literatura, os resultados foram comparados com as soluções analíticas o qual mostrou estar cm concordância. Para o escoamento muma expansão brusca, os resultados foram comparados com os dados numéricos obtidos pelo FreeFlow-2D, os quais mostrou estar em concordância. Também foi realizadas simulacôes para o problema da contraģăo 4:1 e um problema com geometria curva, os quais se mostraram satisfatórios.

Em geral, pode ser concluído que as simulaçeses com o código limFlow-2D, foram satisfatórias. Fi uma vantagem dessa metodologia atual é a sua habilidade em simular escoamentos em geometria complexa.

Acredita-se que esses resultados podem ser melhorados pela inclusão de métodos alternativos para a discretização de condições de contorno de saída de fluido e condiçóes de contorno da equação de Poisson.

A extensão do UmFlow-2D para simular problemas envolvendo superfícies livres é $1 \mathrm{~mm}$ dos objetivos para continuidade deste trabalho. Escoamento de fluidos não Newtonianos o viscoelásticos também estão entre os objetivos para extensão do LmFlow-2D.

Parte deste trabalho estão no IX Simpósio de 'Teses e Disscrtações, ICMC- ISSP São Carlos - SP e foi submetido ao MECOM-2005, VIII Congreso Argentino de Mecánica Computacional, cujo título è "A New Meshfree Approach". 


\section{Referências Bibliográficas}

|Amsden and Harkow, 1970| Amsden, A. and Harlow, F. (1970). The smac method: a numerical technique for calculating incompressible fluid flow. Los Alamos Scientific Laboratory, Report LA, page 4370.

|Aris, 1962| Aris, R. (1962). Vectors, tensors, and the basic equation of Juid mechanics. Dover.

|Armfield and Street, 2002| Armfield, S. and Street, R. (2002). An analysis and comparison of the time accuracy of fractional-step methods for the navier-stokes equations on staggered grids. International Journal for Numerical Methods in Fluids, 38:255-288.

|Ayachour, 200:3| Ayachour, E. (2003). A fast implementation for gmres method. Journal of Computational and Applicd Mathemalies, 159:269-283.

|Batchelor, 1970| Batchelor, G. (1970). An Introduction to Fluid Iynamics. Cambridge: University Press, Cambridge.

|Bentley, 1986| Bentley, J. (1986). Programming P'earls. Addison-Wesley, Reading, MA.

|Brandi, 2005| Brandi, A. C. (2005). Estratégias "upunind" e modelagem $\kappa$-є para simulaçăo numérica de escomantos com superfícies livres em altos nimcros de Reynolds. Dissertação de mestrado.

|Castelo et al., 2000| Castelo, A., lomé, M., C.N.L.César, S.McKee, and Cuminato, J. (2000). Freeflow: An intrgrated simulation system for three-dimensional free surface flows. Computing and Visualization in Science, 2:199-210.

|Castro, 1995| Castro, .. B. (1995). Introdução à engenharia de requisitosl,. In: XV Congresso da Sociedade Brasileira de Computação, JAI'95, page 43.

|Chapra and Canale, 1990| Chapra. S. C. and Canale, R. P. (1990). Numerical Methods for Enginears, 2nd ed. MeGraw-Hill International Editions, Applied Mathematics Series.

|Chorin, 1968| Chorin, A. (1968). Numerical solution of the navier-stokes equations. Mathematics of Computation, 2:745-762.

|Courant et al., 1967| Courant, R., Friedrichs, K., and Lewy, H. (1967). On the partial differential equations of mathematical physics. IBM Journal Research and Developmenl, 11:215-231. 
|Cuminato and Meneguette, 1999| Cuminato, J. $\Lambda$. and Meneguette, M. (1999). Discretização de Equações Difcrenciais Parciais: Técnica de Diferenças Finitas. Em preparação.

|Cunha, 2000| Cunha, M. C. C. (2000). Métodos Numéricos. Editora Unicamp, Campinas.

|Doricio, 2003| Doricio, J. I. (2003). GENSMAC-SOF: Um mélodo numérico para simular escoamentos incompressiveis de fluido de segunda ordem. Biblioteca Digital de Teses e Dissertações-www.teses.usp.br, ICMC-USP-São Carlos, Brasil.

|Ferreira, 2001| Ferreira, V. G. (2001). Análise e implementaçăo de esquemas de convecção e modelos de turbulência para simulação de escoamentos incompressiveis envolvendo superfícies livres. Lniversidade de São Paulo, Tese de Doutorado, ICMC-LSP.

|Ferziger and Peric, 1999| Forziger, J. H. and Peric, M. (1999). Computational Methods for Fluid Dynamics. 2. Heidelberg, Springer-Verlag.

|Flecther, 1992| Flecther, C. A. (1992). Computational Techniques for Fluid Flows. Heidelberg, Springer-Verlag.

|Fletcher, 1975| Fletcher, R. (1975). Numerical Analysis Dundee. Springer-Verlag- Lectures Notes in Mathematics, vol.506.

|Fortuna, 2000| Fortuna, A. O. (2000). Técnicas Computacionais para Dinâmica de Fluidos Conceitos Teóricos e Aplicações. Edusp, São Paulo.

|Gamma et al., 2000| Gamma, E.;Helm, R., R., J., and Vlissides, J. (2000). Padrões de. projeto: soluçócs reutitizáveis de sofiware orientado a objelo. Bookman.

|Golub and Loan, 1989| Golub, G. H. and Loan, C. F. V. (1989). Matrix Compulations, 2nd e. Baltimore: Jonhs Hopkins Universaty Press.

|Gossler, 2001| Gossler, A. (2001). Moving least-squares: A numerical differentation methods for irregularly spaced calculation points. Sandia technical Report, SAND2001.

|Gresho, 1990| Gresho, P. M. (1990). On the theory of semi-implicit projection methods for viscous incompressible flow and its implemention via a finite element method that also introduces a nearly consistent mass matrix. International Journal Jor Numerical Method in Fluid, 11:587-620.

|Gresho and Chan, 1990| Gresho, P. M. and Chan, S. T. (1990). (On the theory of semi-implicit projection methods for viscous incompressible flow and its implemention via a finite element method that also introduces a nearly consistent mass matrix. part 2 : Implementation. International Journal for Numerical Method in Fluid, 11:621 659.

|Griebel et al., 1998| Griebel, M., Dornscifer, T., and Neunhoeffer, T. (1998). Numerical Simulation in Fluid Dynamics: A Practical Introduction. Society for Industrial and Applied Mathematics - SIAM.

|Grossi, 2003| Grossi, L. (2003). Desenvolvimento de métodos numéricos para a simulação de escoamentos não-neutonianos e viscoelástico com superfície livre. Biblioteca Digital de Teses e Dissertações-www.teses.usp.br, ICMC-LSP-São Carlos, Brasil. 
|Harlow and Welch, 1965| Ilarlow, F. and Wclch, J. (1965). Numerical calculation of time-dependent viscous incompressible flow of fluid with a free surface. Phys. Fluids, pages 8:2182-2189.

|Hirt and Cook, 1972| Hirt, C. and Cook, J. (1972). Calculating three-dimensional flows around structure and over rough terrain. Journal of Computational Physics, 10:324-340.

|Hirt and Nichols, 1971| Hirt, C. and Nichols, B. (1971). Improved frec surface boundary-conditions for numerical incompressible-flow calculations. Journal of Computational Physics, 8:431-148.

|IEEE, 1994| IEEL (1994). IEEE Standards Collection. Software Engineering. IFEF, New York - NY.

|Jankowski, 1998| Jankowski, J. (1998). A non-hydrostatic model for free surface flows. Universitat Hannover.

|Jensen, 1972| Jensen, P. S. (1972). Finite difference techniques for variable grids. Computers and Structures, pages 2:17-29.

|Knuth, 1968| Knuth, D. (1968). Fundamental Algorithms - The Art of Computer Programming. Addison-Wesley, Reading, MA.

|Lanczos, 1952| Lanczos, C. (1952). Solution of systems of Jinear equations by minimized iteration. Journal Res. Nat. Bur. Standards, 49:33-53.

|Lapidus and Pinder, 1982| Lapidus, L. and Pinder, G. F. (1982). Numerical Solution of Partial Differential Equalions in Science and Engineering. A Wiley-Interscience Publication, New York.

|Liska and Orkisz, 1980| Liska, 'T. and Orkis», J. (1980). The finite difference method at arbitrary irregular grids and its application in applied mechanics. Computers and Structures, pages 11:83-95.

|Lizier et al., 2003| Lizier, M. A. S., Nonato, L. G., Castelo, and A. Oliveira, M. C. F. (2003). A topological aproach for detecting and extracting 2 d object models from images,. In: WICCGPI, SIBGRAPI 2003, São Carlos - SP, pages 10-17.

|Lio and Haussler, 2002| Luo, Y. and Haussler, U. (2002). A general finite-difference method based on minimizing global residual. Comput. Methos Appl. Mech. Engrn, pages 191:1421-1438.

Maliska, 1995| Maliska, C. A. (1995). Transferência de Calor e Mecânica dos Fluidos Computacional. LTC Editora - Rio de Janciro.

|Marshall and Grand, 1997| Marshall, J. and Grand, J. R. (1997). A lagrangian vorticity collocation method for viscous, axisymmetric flows with and without swirl. J. Comput. Phys, 138:302-330.

|Mayrhauser, $1990 \mid$ Mayrhauser, A. V. (1990). Software engineering: Methods and management. Academic Press. 
|Miyata and Masuko, 1985| Miyata, H. and Masuko, A. (1985). Finite-difference simulation of nonlinear waves generated by ships of arbitrary threc-dimensional configtration. Journal of Computational Physics, 60:391 436.

|Niceno, 2001| Niceno, B. (2001). Fasymesh: a free two-dimensional quality mesh generator based on delaunay triangulaton.

|Oliveira, 1999| Oliveira, J. (1999). Desenvolvimento de um Sistema de Simulação de Escoamentos de Fluidos com Superfícies livres Bidimensionais. Dissertação de mestrado, ICMC-USP.

|Paiva, 2004| Paiva, D. M. B. (2004). Modelo de Qualidade de Software para Ambiente. Acadêmico Envolvendo Elementos de Design Rationale. Instituto de Ciências Matemáticas e de Comprutação - USP, Dissertação de mestrado, ICMC-USP.

|Panton, 1981| Panton, R. L. (1984). Incompressible flow. John Wiley \& Sons.

|Patankar, 1980| Patankar, S. (1980). Numerical IIeat Transfer and Fluid Flows. Hemisphere Publishing Co.

|Patankar and Spalding, 1972| Patankar, S. and Spalding, D. (1972). A calculation procedure for heat, mass and momentum transfer in three-dimensional parabolic flow. International Journal of Heat and Mass Transfer, 15:1787-1806.

|Perot, 1993| Perot, J. B. (1993). An analysis of the fractional step method. Journal of Computalional Physics, 108:51-58.

|Perrone and Kao, 1975| Perrone, N. and Kao, R. (1975). A general finite difference method for arbitrary mesh. Computers and Structures, pages 5:617-664.

|Peyret and Taylor, 1983| Peyret, R. and Taylor, T. D. (1983). Computational Methods for Fluid Flow. Springer-Verlag.

|Peña, 2003| Peña, D. (2003). Método de diferenças finitas gencralizadas por mánimos quadrados. Dissertação de mestrado, ICMC-USP.

|Phillips and Williams, 2001| Phillips, T. and Williams, A. (2001). A semi-lagrangian finite volume method for newtoniano contraction flows. SIAM Journal on Scientific Computing, pages $22(6): 2152 \cdot 2177$.

|Press et al., 1992| Press, W. H., Flannery, B. P., Teukolsky, S. A., and Vetterling, W. T. (1992). Numerical Recipes in C : The Art of Scientific Computing, 2nd ed. Cambridge University Press.

|Pressman, 1994| Pressman, R. S. (1994). Software Engineering: a practitioner's approach. European Edition, Rio de Janeiro.

|Pressman, 2002] Pressman, R. S. (2002). Engenharia de Software. McGraw-Hill, Rio de Janeiro, five edition.

|Quarteroni et al., 2000| Quarteroni, A., Sacco, R., and Saleri, F. (2000). Numerical Mathematics. Springer. 
|Sabatini, 2002| Sabatini, M. H. (2002). Expansão do sistema FREEFLOW3D para escoamentos com influência a temperatura. Biblioteca Digital de Teses o Dissertações-www.teses.usp.br, ICMC-LSP-São Carlos, Brasil.

|Santos, 2002| Santos, F. (2002). Simulaşão numérica de escoamentos multifásicos utilizando o sistema Freffiou-2D. Biblioteca, ICMC-USP-São Carlos, Brasil, Dissertação de mestrado, ICMC-USP.

|Shepard, 1968| Shepard, D. (1968). A two dimensional function for irregualarly spaced datia. ACM National Conference.

|Silva, 2003| Silva, G. F. (2003). Simulação numérica de escoamentos viscoclástico com. superficie livre usando o ambiente FREEFLOW2D. Biblioteca Digital do Teses e Disscrlações-www.teses.usp.br, ICMC-USP-São Carlos, Brasil.

|Silva, 2005| Silva, M. A. (2005). Desenvolvimento de um método númerico para simular escoamentos viscoelásticos axissimêtricos com superfícies livres. Dissertação de mestrado, ICMC-USP.

|Siquieri, 2002| Siquieri, R. (2002). Análise e implementação de modelos Não-Newtonianos no sistema Freffou-2D. Universidade de São Paulo, Dissertação de mestrado, ICMC-USP.

|Sod, 1989| Sod, G. A. (1989). Numerical Methods in Fluid Dynamics-Initial and Initial Boundary-Value Problems. Cambridge, New York.

|Sousa and Mangiavacchi, 2002| Sousa, F. and Mangiavacchi, N. (2002). Simulaçăo Numérica de Escoamentos Multifásicos Usando o Sistema FreeFlou-3D. Dissertação de mestrado, ICMC-USP.

|Stuart and Dochan, 1991| Stuart, E. R. and Dochan, K. (1991). An upwind differencing scheme for the incompressible Navier-Stokes cquations, volume 8. em Applied Numerical Mathematics.

|Thomas, 1995| Thomas, J. W. (1995). Numerical Partial Differential Equation.s. Springer.

|Tomé and McKec, 1994| Iomé, M. F. and McKee, S. (1994). Gensmac: A computational marker-and-cell method for free surface flows. Journal of Computational Physics, 110:171-186.

|Turine and Masiero, 1996| Turine, M. A. S. and Masicro, P. C. (1996). Especificação de Requisitos: Uma Introdução. Relatório T'écnico, ICMC-USP-São Carlos, SP.

|Urena et al., 2001. Urena, F., Benito, J. J., and Alvare\%, R. (2001). Método adaptativo para la resolución de ecuaciones diferenciales en derivadas parciales de segundo ordem utilizando diferencias finitas generalizadas. Aplicadou a Mathematical Modelling., page ref: $\mathrm{MC} / \mathrm{EM} / \mathrm{AMM} .2884$.

|Viecelli, 1969| Viecelli, J. (1969). A method for including arbitrary external boundaries in the mac incompressible fluid computing technique. Journal of Computational Physics, $4: 54: 3551$. 
|Vorst, 1992| Vorst, H. V. D. (1992). Bi-cgstab: a fast and smoothly converging variant of bi-cg for the solution of nonsymmetric linear systems. SIAM Journal Sci. Statist. Comput., 13:631 644.

|Watson, 1976| Watson, G. (1976). Numerical analysis. Lecture Notes in Mathematics, Springer, Berlin, 506:73-89. 